Florida International University FIU Digital Commons

\title{
Examining Students' Representation Choices in University Modeling Instruction
}

Daryl McPadden

Florida International University, dmcpa001@fiu.edu

DOI: $10.25148 /$ etd.FIDC004079

Follow this and additional works at: https:// digitalcommons.fiu.edu/etd

Part of the Curriculum and Instruction Commons, and the Other Physics Commons

\section{Recommended Citation}

McPadden, Daryl, "Examining Students' Representation Choices in University Modeling Instruction" (2018). FIU Electronic Theses and Dissertations. 3658.

https://digitalcommons.fiu.edu/etd/3658 


\title{
FLORIDA INTERNATIONAL UNIVERSITY \\ Miami, Florida
}

\section{EXAMINING STUDENTS' REPRESENTATION CHOICES IN UNIVERSITY MODELING INSTRUCTION}

\author{
A dissertation submitted in partial fulfillment \\ of the requirements for the degree of \\ DOCTOR OF PHILOSOPHY \\ in \\ PHYSICS \\ by \\ Daryl R. McPadden \\ 2018
}


To: Dean Michael R. Heithaus

College of Arts, Sciences, and Education

This dissertation, written by Daryl R. McPadden, and entitled Examining Students' Representation Choices in University Modeling Instruction, having been approved in respect to style and intellectual content, is referred to you for judgment.

We have read this dissertation and recommend that it be approved.

$\begin{array}{r}\hline \text { Geoff Potvin } \\ \hline \text { Laird Kramer } \\ \hline \text { Pete Markowitz } \\ \hline \text { Eric Brewe, Major Professor }\end{array}$

Date of Defense: March 20, 2018

The dissertation of Daryl R. McPadden is approved.

Dean Michael R. Heithaus College of Arts, Sciences, and Education

Andrés G. Gil Vice President for Research and Economic Development and Dean of the University Graduate School

Florida International University, 2018 


\section{ACKNOWLEDGMENTS}

This page is formally here to thank those who have helped in the process of obtaining the graduate degree. It seems impossible to name everyone who has helped me to this point, but these people deserve all the credit I can possibly give them.

First, I would like to thank my committee members Eric Brewe, Geoff Potvin, Laird Kramer, and Pete Markowitz for the time and effort they took to make this dissertation a reality. I would also thank the Physics Education Research group at Florida International University, past and present, for their support, contributions, and feedback on this work - particularly Geoff Potvin and Jianlan Wang, who allowed me to collect data in their classrooms. This work was also generously supported by two National Science Foundations Grants: \# DUE 1140706 and \#DGE 1038321.

More personally, I would like to express my deepest gratitude to Eric Brewe for always encouraging me to take advantage of and seek out every opportunity available. You gave me the freedom to expand who I am as a researcher, as a teacher, and as a member of this community - I cannot thank you enough for this. To Seth Manthey, thank you for challenging me, pushing me to grow, and teaching me that my experience is not normal. Without your mentorship, feedback, and support, I wouldn't be half the researcher I am today, and this project would not exist as it does. To Jason Dowd, thank you for collaborating and thinking with me on the many side projects that this dissertation has opened up. Your support, flexibility, and accountability have shown me what it means to collaborate and always helped me make progress.

To Vince Kuo and Pat Kohl, thank you for introducing me to teaching, for showing me the magic of $\mathrm{E} \& \mathrm{M}$, and for still continuing to answer my questions. You taught 
me to look beyond content and gave me the instincts that I rely on everyday. Truly, you laid the foundation of all this work. I could not have gotten here without you. I hope you know that the price of ruining my life is endless questions over pho.

To those in the broader PER community, thank you for helping me become a part of this wonderful group of people and teaching me what it means to lead. Particularly to Vashti Sawtelle, Danny Caballero, Paul Irving, J.T. Laverty, Ellie Sayre, Rachel Scherr, Sam McKagan, Paula Heron, Kara Gray, Amy Robertson, Lane Seely, Peter Schaffer, Donna Messina, Ben Van Dusen, Jayson Nisson, Carolina Alvarado, Cassandra Paul, Benedikt Harrer, Noah Finkelstein, Steve Pollock, Bethany Wilcox, Kathy Harper, Adrienne Traxler, and Natasha Holmes: thank you for opening your doors, inviting me to visit, and giving me feedback on this work. It is all the better from your input. Especially to the PERL folks at MSU, I would not have survived writing without you all and the endless shenanigans.

Finally, I want to thank my family. To Carly, Brent, Pete, and Isaac, thank you for always being there, for sending me memes, and for always waiting for me to go see Star Wars/Marvel movies. I would not have gotten through this without you. To my dad, who taught me to listen for the question not being asked, and to my mom, who taught me what it means to be strong - I would not be anywhere without your love and support. I cannot even begin to name your contributions to who I am and what you've done to help me get to this point. Thank you. 


\title{
ABSTRACT OF THE DISSERTATION \\ EXAMINING STUDENTS' REPRESENTATION CHOICES \\ IN UNIVERSITY MODELING INSTRUCTION
}

by

\author{
Daryl R. McPadden \\ Florida International University, 2018 \\ Miami, Florida \\ Professor Eric Brewe, Major Professor
}

Representations (such as pictures, diagrams, word descriptions, equations, etc.) are critical tools for learning, problem solving, and communicating in science, particularly in physics where multiple representations often serve as intermediate steps, a means to evaluate a solution, and highlight different aspects a physical phenomenon. This dissertation explores the representation choices made by students in the University Modeling Instruction (MI) courses on problems from across introductory physics content. Modeling Instruction is a two-semester introductory, calculus-based physics sequence that was designed to guide students through the process of building, testing, applying, and refining models. As a part of this modeling cycle, students have explicit instruction and practice in building, evaluating, and coordinating representations in introductory physics. Since I am particularly interested in representations across all of introductory physics, this work was situated in the second semester of MI. To address students' representation choices, the Problem Solving and Representation Use Survey (PSRUS) was developed as modified card sort survey, which asked students to simply list the representations that they would use on 25 physics questions from across introductory physics. Using non-parametric statistical tests 
(Mann-Whitney-Wilcox, Wilcoxon-Ranked Sign, and Cliff's Delta), I compare the number and variety of representations that students choose. Initially, students who took the first semester of MI use significantly more representations in their problem solving when compared to those who did not; however, there are significant gains in the number of representations that these students choose over the semester across the introductory physics content. After significant changes to the second semester MI curriculum, the difference between these two groups disappears, with both groups increasing their representation choices when compared to the previous semester. Using network analysis to compare students' concurrent representation choices, I also show that students use a consistent set of representations on mechanics problems; whereas, they choose a wider variety on electricity and magnetism (EM) problems. In both mechanics and EM, pictures serve as an important connecting representation between the others. I use these results to make suggestions for instructors, curriculum developers, and physics education researchers. 


\section{TABLE OF CONTENTS}

CHAPTER

PAGE

1 INTRODUCTION 1

1.1 Classroom Context . . . . . . . . . . . . . . . . 5

1.2 Guiding Research Questions . . . . . . . . . . . . . . . . . 9

1.3 Structure of Dissertation . . . . . . . . . . . . . . . . . . . 10

2 IMPACT OF THE SECOND SEMESTER UNIVERSITY MODELING INSTRUCTION COURSE ON STUDENTS' REPRESENTATION CHOICES 13

2.1 Abstract . . . . . . . . . . . . . . . . . . . . . 13

2.2 Introduction . . . . . . . . . . . . . . . . . . . . . . 14

2.3 Modeling Instruction $\ldots \ldots \ldots \ldots$

2.4 Research Questions . . . . . . . . . . . . . . . . . . . . . . . . . . . . 21

2.5 Methods . . . . . . . . . . . . . . . . . . . . . . 21

2.5.1 Problem Solving and Representation Use Survey (PSRUS) . . 21

2.5.2 Data Collection . . . . . . . . . . . . . . . . . 25

2.5 .3 Data Analysis . . . . . . . . . . . . . . . . . . 26

2.6 Results and Discussion . . . . . . . . . . . . . . . . . . . . . . 30

2.6 .1 Baseline - New vs returning . . . . . . . . . . . . . . 31

2.6 .2 Returning Students . . . . . . . . . . . . . . . . . . . . 33

2.6 .3 New Students . . . . . . . . . . . . . . . . . . . . . . . . . . 39

2.7 Conclusions . . . . . . . . . . . . . . . . . . . . . . . . . . . . . . . . 41

3 EXTENDING UNIVERSITY MODELING INSTRUCTION TO INTRODUCTORY ELECTRICITY AND MAGNETISM 47

3.1 Abstract . . . . . . . . . . . . . . . . . . . . 47

3.2 Introduction $\ldots \ldots \ldots \ldots \ldots \ldots \ldots$

3.3 Literature Review . . . . . . . . . . . . . . . . . . . . . . . 51

3.3.1 Modeling Instruction Theoretical Framework . . . . . . . . . . 51

3.3 .2 Use of Multiple Representations . . . . . . . . . . . . . . . . 58

3.4 Curriculum Changes . . . . . . . . . . . . . . . . . . . . . . 61

3.4 .1 Changes made to EM . . . . . . . . . . . . . . . . . . . 62

3.4 .2 Changes made to Mechanics . . . . . . . . . . . . . . . . 67

3.5 Research Questions . . . . . . . . . . . . . . . . . . . . . 68

3.6 Methods . . . . . . . . . . . . . . . . . . . . . . . . . . . 68

3.6 .1 PSRUS Survey . . . . . . . . . . . . . . . . . 68

3.6.2 Data Collection . . . . . . . . . . . . . . . . . . . . . . 72

3.6 .3 Analysis . . . . . . . . . . . . . . . . . . . . . . 73

3.7 Results and Discussion . . . . . . . . . . . . . . . . . . . 78

3.8 Conclusions . . . . . . . . . . . . . . . . . . . . 85 
4 EXAMINING CONCURRENT REPRSENTATION CHOICES IN

UNIVERSITY MODELING INSTRUCTION 89

4.1 Abstract . . . . . . . . . . . . . . . . . . 89

4.2 Introduction . . . . . . . . . . . . . . . . . . . . . . . 90

4.3 Study Context . . . . . . . . . . . . . . . . . . . 94

4.4 Research questions . . . . . . . . . . . . . . . . . . . . . . 97

4.5 Data Collection . . . . . . . . . . . . . . . . . . . . . 98

4.6 Network Analysis . . . . . . . . . . . . . . . . . . . . . . . . 102

4.6.1 Building the Network . . . . . . . . . . . . . . . . 102

4.6 .2 Network Filter . . . . . . . . . . . . . . . . . . . 106

4.6.3 Edge Betweenness Centrality . . . . . . . . . . . . . . . 107

4.7 Results . . . . . . . . . . . . . . . . . . . . 108

4.8 Conclusions . . . . . . . . . . . . . . . . . . . . 113

5 CONCLUSION 118

5.1 Implications and Intellectual Merit . . . . . . . . . . . . . . . 121

5.2 Limitations of the PSRUS . . . . . . . . . . . . . . . . . . . . 123

5.3 Future Directions . . . . . . . . . . . . . . . . 125

$\begin{array}{ll}\text { REFERENCES } & 128\end{array}$

$\begin{array}{ll}\text { APPENDICES } & 139\end{array}$

$\begin{array}{ll}\text { VITA } & 168\end{array}$ 


\section{LIST OF FIGURES}

FIGURE

PAGE

1 Example of student's model in mechanics . . . . . . . . . . . . . . 18

2 Representations given on the PSRUS . . . . . . . . . . . . . 22

3 Sample distribution of PSRUS responses . . . . . . . . . . . . . . 27

4 Summary of medians from PSRUS results . . . . . . . . . . . . . 31

$5 \quad$ Cliff's Delta effect sizes comparing new and returning students . . . . 32

$6 \quad$ Cliff's Delta effect sizes comparing pre- to post-mechanics responses . 34

$7 \quad$ Cliff's Delta effect sizes comparing pre- to post-EM responses . . . . 36

8 Cliff's Delta effect sizes comparing mechanics to EM . . . . . . . 38

$9 \quad$ Box plots comparing pre to post on EM questions for new students . 42

10 Box plots comparing pre to post on mechanics questions for new students 43

11 Content comparison of MI-EM between Spring 2015 and Spring $2016 \quad 64$

12 Representations given with the PSRUS . . . . . . . . . . . . . . 70

13 Sample distribution from PSRUS responses . . . . . . . . . . . . . . . 74

14 Summary of PSRUS results across MI-EM development . . . . . . . . 80

15 Cliff's Delta effect sizes across MI-EM curriculum development . . . . 84

16 Example of student's model in EM . . . . . . . . . . . . . 96

17 Representations given on the PSRUS . . . . . . . . . . . . . . . 100

18 Process for building the representation networks . . . . . . . . . . . . 104

19 Filtered networks for mechanics and EM . . . . . . . . . . . . 109 


\section{ABBREVIATIONS AND ACRONYMS}

A - Assumptions

Bars - Energy Bar Charts

CD - Circuit Diagrams

EM - Electricity and Magnetism

Eq - Equations

FBD - Free Body Diagram

FL - Field Lines

Gr - Graphs

MDM - Modeling Discourse Management

MI - University Modeling Instruction

MI-EM - University Modeling Instruction Electricity and Magnetism course

MI-Mech - University Modeling Instruction Mechanics course

MM - Motion Map

MTI - Modeling Theory of Instruction

MTS - Modeling Theory of Science

Oth - Other

PER - Physics Education Research

Pic - Picture

Pies - Energy Pie Charts

PL - Potential Lines

PSRUS - Problem Solving and Representation Use Survey

PV - Momentum Vectors

RHR - Right Hand Rules 
SS - System Schema

Wd - Words

WMW - Wilcoxon-Mann-Whitney test

WSR - Wilcoxon-Signed-Rank test 


\section{INTRODUCTION}

When thinking about or trying to understand a complex phenomenon, representations (such as equations, word descriptions, pictures, graphs, diagrams, etc.) become important tools for highlighting and coordinating disparate features. While the contexts may vary widely, common representations (like those listed above) serve to facilitate communication, visualization, and construction of knowledge from the introductory, classroom level to peer-reviewed, scientific work.

In science, the use of multiple representations is often encouraged (or required) to make predictions, explain why a process occurs, and/or to describe the relevant features of a phenomena $[1,2,3,4]$. These processes and representations are just as relevant in the science classroom as they are in scientific fields. In the classroom context, representations refer to the external tools that students create and use in their problem solving (as opposed to the internal, mental visualizations that students may be constructing) $[5,6]$. For example, a student in an introductory physics course might draw a picture and create a force diagram before using an equation to calculate the acceleration of charged particle. We would refer to the picture, the force diagram, and the equations that student used as the representations (or tools) that the student used to get their solution.

Particularly in physics, multiple representations can serve a variety of purposes in problem solving (for students and scientists alike) [5, 7]. For instance, many representations serve complementary roles, meaning they communicate much of the same information but in different ways or emphasizing a different aspect of that information [5]. One such example would be that you could create a table, an equation, or a 
graph to represent the same set of data. In the classroom, using complementary representations can be especially productive for students by providing multiple pathways to a solution. If a student does not understand or remember an equation, perhaps they can construct a graph to get to their answer. Alternatively, complementary representations can help draw students' attention to different salient features [5]. From a graph, it can be difficult to tell if there are missing data from a set, which is strikingly obvious from missing rows in a table. Conversely, it is extremely difficult to see a consistent pattern in a table of numbers, whereas the pattern (or trend of the data) is often the first feature you notice in a graph. Often the goal in the classroom is to have students reason about both these features (missing data and trends in data) and having multiple complementary representations to rely on can help students in this endeavor.

In contrast to complementary roles where representations are used in parallel, multiple representations can also be used as intermediate steps (or sequentially), where the construction of one representation then dictates part of the composition of the next. Ainsworth refers to using sequential representations as "constraining representations" [5]. For example, physics students are usually told to begin a problem by drawing a picture, which helps students identify the objects and how they are related spatially. The next step could involve drawing a system schema [8], where the objects are now represented by circles (rather than their shape) and the interactions between those objects represented by double headed arrows. In drawing the system schema, students build off of what they drew in the picture, but are now abstracting prominent features and interactions. From the number of interactions between objects, students can draw a force diagram (with each interaction corresponding to a 
force), and use the force diagram to set up Newton's Second Law equation to solve for the acceleration of an object. In each step of the process, the representations have overlapping information (which helps students coordinate between those representations), but also build on to what was done before. By dividing the information in the problems, multiple representations provide intermediate steps for students in their problem solving, reduce the cognitive load of problem solving, and help students move from a familiar representation (like a picture) to one that is more abstract (like a force diagram or an equation) $[5,9,10,11,12]$.

Finally, multiple representations can also be used as a means to construct deeper understanding [5]. For example, energy pie charts were developed to help students reason about the energy in a system, including how energy is stored in the system, how energy is transferred, and energy conservation across the system boundary [13]. Through creating a series of pie charts, students can track how the energy changes over time in the system and explain how those changes impact the objects in the system. Energy pie charts can also be used to construct an equation for any two states of the system (following energy conservation) and are fundamentally tied to the choice of system (and thus representations like the system schema). While students may not need to construct the energy pie charts to reach an energy conservation equation, the pies provide a tool for students to justify their work using conceptual reasoning about the forms, transfer and conservation of energy. Particularly in physics, there are many representations that are designed to emphasize conceptual reasoning derived from fundamental physical laws before building to equations or other quantitative representations (e.g., force diagrams, momentum vectors, power boxes [14], potential lines, field lines). 
However, many researchers have shown that using representations in these ways is not automatic and is a skill that needs to be developed $[4,15,11,16,17,18]$. Both in studies of multiple representation use and the use of a single representation, there have been consistent results that show expert physicists use representations differently than students $[6,19,20]$. Kohl and Finkelstein show that experts are able to more easily switch between representations and are flexible in their starting point, whereas students struggle to move between representations and use more representations overall [19]. Hecker and Kuo, Hallinen, and Conlin found that students actually did worse on force problems when prompted to draw a force diagram and that prompting certain representations can cue students to simply follow procedures rather than deep conceptual reasoning $[21,22]$. Other studies indicate that the type of representation given in a problem strongly dictates the solution path that students take, which further supports this argument [23, 24, 25]. These studies indicate that if we want students to be able to use, coordinate and reason effectively with multiple representations, they require sufficient time, scaffolding, and practice with those representations.

To address these concerns, University Modeling Instruction - commonly referred to as Modeling Instruction - has incorporated multiple representation use and coordination into the curriculum as a part of the model building process. Modeling Instruction (MI) is a two-semester course sequence for introductory, calculus-based physics, which is centered around students building, testing, applying, and refining models. The first semester in the sequence focuses on the models in introductory mechanics (MI-Mech), while the second semester focuses on those in introductory electricity and magnetism (MI-EM). As a part of constructing and evaluating their 
models, the MI curriculum focuses heavily on how each representation is introduced, how representations coordinate with one another, and practice in using and applying multiple representations. Because MI courses have an explicit focus on representation use, these courses provide an interesting context to study how students use representations in introductory physics.

Since multiple representations serve such an important role in physics, it is important to understand what representations students are finding useful, the variety of representations that students choose in different contexts, and what representations students choose to use concurrently on physics problems. Through examining students' representation choices in the MI course, this dissertation shows insights into students' problem solving, the impacts of context on their representation use, and implications for teaching and curriculum design.

\subsection{Classroom Context}

Modeling Instruction (MI) is a reformed, active-learning classroom that is designed to guide students through constructing, testing, applying and refining conceptual models of physical phenomena [26]. In the context of the MI courses, a conceptual model is defined as a purposeful, coordinated set of representations of a particular class of phenomena that exists in the shared domain of discourse and is an inscription of disciplinary knowledge $[27,2]$. Instructionally, this model definition means that a model is composed of a coordinated set of representations that together have explanatory power, predictive ability, and descriptions of salient features of that phenomenon $[2,1]$. The day-to-day activities of the MI courses then are spent building up each 
of the pieces of each model in the course, with multiple representations serving the fundamental base of the model.

The curricula for the MI courses were designed using the Modeling Instruction framework, which includes the Modeling Theory of Science (MTS), Modeling Theory of Instruction (MTI), and Modeling Discourse Management (MDM). The MTS avers that science is the process of developing, testing, applying, and refining models and that this process is what it means to be "doing science" [28]. As practicing scientists, this is what we do everyday - from famous physicists like Newton and Maxwell $[28,29]$ to the scientific practices outlined in the Next Generation Science Standards and laboratory practices $[30,31]$. The MTI then states that if science is the practice of developing, testing, applying, and refining models, then students should be developing, testing, applying, and refining models in their science courses [32, 26, 33]. Brewe outlines how this process occurs in the MI courses through the Modeling $\mathrm{Cy}-$ cle: introduction of phenomenon and representation, coordination of representation, application of the model to a variety of contexts, abstraction and generalization between contexts, and model refinement [26]. The MTI and the Modeling Cycle guide the structure and goals of each activity in the MI courses.

The first two steps of the Modeling Cycle are focused on helping students use and coordinate the representations in their models, which is significant for several reasons. First, students are only introduced to a new representation when it is motivated by the phenomenon and the need to represent an aspect of that phenomenon. Second, there are explicit activities, instruction, and time spent to help students coordinate the various representations and particularly integrate new representations with those that they have already used. Finally, students are practicing and using the representations 
in the last three stages of the modeling cycle as they apply and generate models for a variety of situations. Thus, multiple representations are a critical part of the dayto-day activities and conversations in the MI classroom.

The final piece of the MI framework is the MDM. Modeling Discourse Management is a set of strategies that instructors use to promote students' participation in the modeling process and guides how the classroom structures are carried out [34, 35]. On each activity, students begin by working in small groups of three students. As they reach the end of the activity, students are asked to make a small whiteboard (approximately 2'x3') about what they learned, what rules or patterns they see, and what they still have questions about. Students then come together for a "board meeting," where they have the opportunity to present their findings, compare with the work of other groups, critique each others ideas and come to consensus about what they learned. Ideally, the discussion is led by the students, both in terms of asking, presenting, and answering questions. The role of the instructor is then to facilitate these discussions, to make sure that the key ideas are being brought up, and to step in as a content expert to answer questions if needed.

Following this framework and building off of the Modeling Instruction materials that have been developed for high school physics courses [36], the MI-Mech course has been developed at multiple universities over the last two decades, most recently at Florida International University (FIU) [37]. There have been a number of research studies studying the MI-Mech courses at FIU (taught in both 30 and 75 student formats), which have shown the course to be successful on multiple measures. In addition to larger conceptual gains on conceptual surveys (FCI and FMCE) when compared to lecture, MI-Mech students are more likely to pass the course (have a 
lower fail-drop-withdraw rate) [38, 39, 40]. Furthermore, students in the MI-Mech course have more favorable attitudes towards physics by the end of the course (as measured by the CLASS survey) independent of the instructor that taught the course $[41,42]$. In contrast, there is typically a decrease in favorable attitudes toward physics in introductory physics classes [42, 43]. In addition, students in MI-Mech have an increased participation over the semester and form a larger network of peers when compared to the equivalent lecture course, which contributes to their success in the course [44].

Motivated by the success of the MI-Mech course, the MI-EM course more recently underwent a significant curriculum design to align the MI-EM with the Modeling Instruction framework. As of the Spring 2015 semester, the MI-EM course was taught following the MDM, but not adhering to the MTI and the MTS. Meaning, the MIEM course was taught in a similar style to the MI-Mech course (students working in small groups, creating whiteboards, participating in board meetings); however, the curricular materials did not follow the Modeling Cycle or build towards creating the models in EM. Between the Spring 2015 and Spring 2016 semesters, the MI-EM materials were significantly changed with particular attention paid to aligning the activities with the MTI and the MTS.

The changes made to the MI-EM curriculum over the Spring 2015 and Spring 2016 semesters provided the unique opportunity to study how students' representation choices changed as an effect of messaging and curricular changes made to the materials, which is especially relevant given the focus on representations in the MTI. As the MI-EM course builds off the ideas, content, and classroom culture established in MI-Mech, we were also interested in studying how students' representation choices 
differ between the mechanics and EM contexts. Thus, we chose to conduct this study in the MI-EM course, after students had taken a mechanics physics course as the prerequisite, to be able to study students' representational choices across all of the introductory physics content. Furthermore, researching the second semester of the introductory physics sequence also creates space to study how students who did not take MI-Mech take up the use of representations that are emphasized in MI-EM. While any physics course would necessarily use representations, we would expect the the variety, coordination, and practice with multiple representations to be different between MI and lecture courses. Together these ideas lead to the guiding research questions for this dissertation outlined in the following section.

\subsection{Guiding Research Questions}

Because representations serve as critical tools in science, in physics, and in the MI courses specifically, the dissertation focuses on the following research questions:

1. Prior to the curriculum changes, what are the differences in representation choices between students who took MI-Mech (returning to the MI course format) and those who did not (new to the MI course format), particularly in how many and which representations they choose? How do students' representation choices vary based on physics content (mechanics or EM questions)? How does the MI-EM course impact both new and returning students' representation choices over the course of the semester?

2. Can we see the effect of curricular changes in the number and variety of students' representation choices across the semester? How are the number and variety of 
students' representation choices impacted by the type of mechanics course they took and the context (mechanics or EM) of the problem type?

3. By the end of MI-EM (after the curricular changes), what representations do students see as useful together when problem solving? What differences do we see between students' concurrent representation choices on questions in mechanics and EM contexts?

\subsection{Structure of Dissertation}

This dissertation follows the Florida International University Collected Papers format, with chapters comprised of related papers that have been (or will be) submitted to peer-reviewed, scientific journals. (Note that as each of these papers are submitted to a scientific journal, I use the first person "we" to refer to myself as first author and advisor(s) as subsequent authors.) While together these papers address the research questions outlined in the previous section, they are each self-contained, including sections on motivation, literature review, methods, results, and conclusions.

The first paper (Chapter 2) addresses research question one (listed above) and outlines the development of the Problem Solving and Representation Use Survey (PSRUS), which is also used in the subsequent chapters. Using non-parametric statistics, we analyze the number and variety of representations that both new and returning students use in both EM and Mechanics contexts over the Spring 2015 semester of MI-EM. This analysis serves as the basis of students' representation choices prior to the curricular changes made to MI-EM. This paper, entitled "Impact of the second semester University Modeling Instruction course on students' representation choices" 
was submitted and published in the Physical Review - Special Topics: Physics Education Research in Fall 2017.

The second paper (Chapter 3) addresses research question two (listed above) and describes how the Modeling Instruction framework was extended and applied to the MI-EM course at FIU. In addition to illustrating the changes made to the curriculum, this paper uses non-parametric statistics to compare students' representation choices prior to and after the modifications, exemplifying the evaluation of a curriculum on non-content based learning goals. This paper was submitted to the European Journal of Physics - Focus Collection on Modelling in Physics Instruction in Spring 2018.

The third paper (Chapter 4) addresses research question three (listed above) using network analysis to examine the concurrent representation choices that students make in mechanics and EM. This study uses only data from the Spring 2016 post-PSRUS (after the curriculum modifications) to examine students' problem solving by the end of the two-semester sequence. This paper will be submitted to the Physical Review Special Topics: Physics Education Research.

I conclude with a summary of the three papers and the intellectual merit of the studies, the implications for teaching and curriculum development suggested by this work, the limitations of these studies, and possible future research directions.

In summary, each of the chapters of this dissertation are as follows:

1. Introduction - motivation, theoretical background, and literature review.

2. Impact of the second semester University Modeling Instruction course on students' representation choices. Published in Physical Review - Physics Education Research, Vol. 13, Is. 2 (2017). 
3. Extending University Modeling Instruction to Introductory Electricity and Magnetism. Submitted to the European Journal of Physics - Focus Collection on Modelling in Physics Instruction.

4. Examining Concurrent Representation Choices in University Modeling Instruction. To be submitted to Physical Review - Physics Education Research.

5. Conclusions - summary and intellectual merit of the studies, implications for teaching and curriculum development, limitations, and future directions of research. 


\section{IMPACT OF THE SECOND SEMESTER UNIVERSITY MODELING INSTRUCTION COURSE ON STUDENTS' REPRESENTATION CHOICES}

\section{$2.1 \quad$ Abstract}

Representation use is a critical skill for learning, problem solving, and communicating in science, especially in physics where multiple representations often scaffold the understanding of a phenomenon. University Modeling Instruction, which is an activelearning, research-based introductory physics curriculum centered on students' use of scientific models, has made representation use a primary learning goal with explicit class time devoted to introducing and coordinating representations as part of the model building process. However because of the semester break, the second semester course, Modeling Instruction - Electricity and Magnetism (MI-EM), contains a mixture of students who are returning from the Modeling Instruction - Mechanics course (to whom we refer to as "returning students") and students who are new to Modeling Instruction with the MI-EM course (to whom we refer to as "new students"). In this study, we analyze the impact of MI-EM on students' representation choices across the introductory physics content for these different groups of students by examining both what individual representations students choose and their average number of representations on a modified card-sort survey with a variety of mechanics and EM questions. Using Wilcoxon-Signed-Rank tests, Wilcoxon-Mann-Whitney tests, Cliff's Delta effect sizes, and box plots, we compare students' representation choices from pre- to post-semester, from new and returning students, and from mechanics and EM content. We find that there is a significant difference between returning and new students' representation choices, which serves as a baseline comparison between 
Modeling Instruction and traditional lecture-based physics classes. We also find that returning students maintain a high representation use across the MI-EM semester, while new students see significant growth in their representation use regardless of content.

\subsection{Introduction}

Representations (including word descriptions, equations, pictures, diagrams, etc.) form the foundation of communication and problem solving in science. Within physics, there is a wide variety of representations used, with multiple representations often used or required for a single problem. By casually flipping through any introductory physics textbook, you can easily see a scattering of equations, force diagrams, pictures and graphs within only a few pages [45, 46].

For students, these representations serve as tools to help them learn, understand a problem or phenomena, and evaluate their results by serving a variety of purposes $[47,7]$. Ainsworth has categorized the multiple purposes into three classes: representations that serve complementary roles, representations that constrain interpretation, and representations that help construct deeper understanding [5]. As an example of representations being used in a complementary role, students can often find several pathways to a solution by using multiple representations, like using a graph rather than an equation or table. Using different representations can be especially productive if there are multiple tasks or if students prefer one representation over another [5] since each of these representations (i.e. graph, table, equations) of a data set highlight different salient features of the situation that may not be obvious from the

others. Multiple representations can also aid students by dividing the information 
and thus reducing cognitive load $[11,12]$. By offering more than one means to the correct answer and highlighting different or overlapping information, multiple representations have been shown to increase students' ability to solve problems [48, 12, 49]. In contrast, constraining representations serve a different purpose; their role is to use familiar tools to help students form and understand a more abstract representation, often through an inherent shared property of the representations $[5,9]$. This function of multiple representations is to explicitly coordinate between representations and can serve as an evaluative check of an answer. Furthermore, representations often provide concrete, intermediate steps to help students reason abstractly about a problem and move from reading a problem statement to calculating an answer[5, 10]. In physics, many representations such as free body diagrams or energy pie charts [13] have also been designed to help students conceptually reason about the problem and construct a deeper understanding of the physical phenomenon. By asking students to create a qualitative representation that then builds into quantitative representations, the intent is to help students ground their calculations and solutions in physical meaning. Given these advantages for students, many educators and researchers in physics and STEM more broadly have recommended using multiple representations in the physics classroom to help students learn $[3,50,51,52,26,53,47,54]$.

Within physics and other STEM disciplines, there is extensive research on how students use individual representations. To name a few examples, this includes research on graphs $[55,56,57,58,59]$, equations $[60,61,62]$, force diagrams $[21,51]$, pictures [63], and energy diagrams $[53,64,65]$. In comparison, the research on students using multiple representations is much more sparse. Rosengrant, Etkina, and Van Heuvelen provide a summary of research on multiple representations in Physics 
Education Research [7], which indicates that using multiple representations improves students' conceptual understanding and ability to solve problems $[8,66]$. Traxler et. al. and De Leone and Gire have shown that students in reformed physics classes or inquiry-based classes use more representations than their lecture counterparts [67, 49]. De Leone and Gire go beyond that to say that the students who used representations in addition to mathematical representations tended to be more successful in solving the problem [49].

That being said, representation use is a skill that students develop $[4,11,16,17$, 18]. Kohl and Finkelstein show that while both expert and novice physics problem solvers use multiple representations, they use them in distinctly different ways: experts are able to move between representations more quickly and are more flexible in their starting point, whereas novices use and jump between more representations in total [19]. If students are not given sufficient time, scaffolding, and practice with representations, they can cause confusion and be ineffective for students [4, 15]. Bakker and Derry succinctly state that "if students ostensibly learn knowledge in the absence of reasoning with representations that make sense to them, then this knowledge is likely to become inert and hard to transfer to new situations" (emphasis added) [68]. For example, Heckler showed that students actually did better on certain force problems when they were not prompted to use force diagrams, suggesting students are viewing the representation as a separate task that is unrelated to solving the problem [21]. Building on Heckler's work, Kuo, Hallinen and Conlin found that prompting for specific diagrams prompts students to follow standard problem solving procedures, rather than deep conceptual reasoning about the problem [22]. This is further supported by studies that show that how a question is formulated can dictate 
what representations and solution path students take $[23,24,25]$. These studies indicate that students do not naturally use the representations as their instructors intend. Therefore, it is important that we teach students how and when to use representations in order for them to use multiple representations effectively.

\subsection{Modeling Instruction}

Because representations fill such a critical role in the process of doing science, Modeling Instruction has incorporated explicit class time and instruction on the representations into the curriculum, with their understanding and use as one of the primary learning goals for students in the class. University Modeling Instruction is a researchbased, active-learning curriculum that has been developed and refined over the past ten years at Florida International University (FIU) [37] for introductory, calculusbased physics classes, building on the high school Modeling Instruction materials and

the Modeling Theory of Instruction [50, 28, 32, 26]. At the university level, there are currently curricula developed for the first and second semester of introductory physics: Modeling Instruction - Mechanics (MI-Mech) and Modeling Instruction Electricity and Magnetism (MI-EM).

Both of the Modeling Instruction courses are highly reformed, inquiry-based physics classes that use guided activities, experiments, and discussions to help students build a physical understanding of the world around them with conceptual models (as opposed to a physical or replica models). We have defined these conceptual models to consist of a coordinated set of representations (graphs, equations, diagrams, etc.) with the ability to explain, predict, and describe a set of physical phenomena $[2,1,26]$. For example, when asked to create a model of an accelerating train, we would expect 


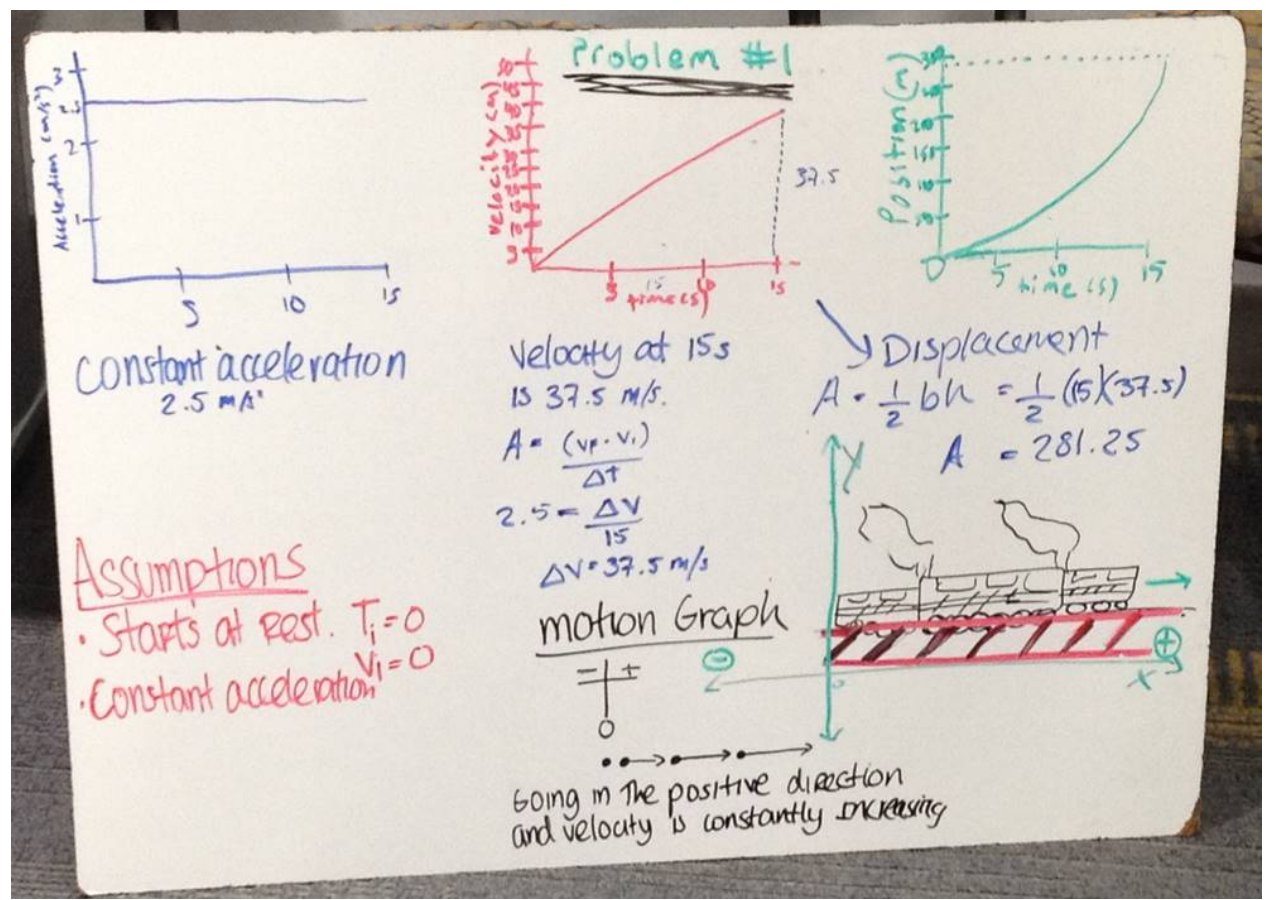

Figure 1: Example of student's model in mechanics.

This is an example of a conceptual model for an accelerating train created by students in the Modeling Instruction - Mechanics course. It shows how a conceptual model consists of a coordinated set of representations including: equations, position/velocity/acceleration graphs, a picture, a motion map, an explicit statement of assumptions, and a word description. 
a solution that looks like the one shown in Figure 1, which contains not only equations, but a picture, word description, motion map, explicit statement about their assumptions, and position/velocity/acceleration graphs.

To create their models in class, students work in groups of three to collect data, solve problems, or derive results; they then summarize their findings, solutions, and remaining questions on a shared whiteboard. Once all of the groups have whiteboards, they come together for a large group discussion (24-30 students) or "board meeting" where they discuss what they found, ask questions to their peers, critique each other's work, and come to consensus about what they learned from the activity. Rather than lecturing, the role of the instructor in these classes is to plan the activities, guide the discussion, and facilitate student work. At Florida International University (FIU), MI-Mech and MI-EM have been implemented in 30 and 75 student formats and have been shown to be successful on multiple measures [38, 41, 69, 44, 70].

For each semester of Modeling Instruction, the curriculum is centered around 4-5 general conceptual models based on a class of phenomena, rather than 10-12 textbook chapters [26]. As described by Brewe, the course generally follows a model building cycle, which repeats approximately every two weeks: introduction and representation of a phenomenon, coordination of representations, application of the model to a variety of contexts, abstraction and generalization of the model, and finally refinement of the model [26]. This means that in class, Modeling Instruction students are using multiple representations early and often, adding representations over the course of the semester as they become needed and applicable to the model that students are building. While traditional physics classes (lecture, lab, recitation courses) certainly use, require, and teach representations as a part of the curriculum, it is rarely an 
explicit learning goal for the course or given specific instruction. Often a detailed discourse about how to use the representation, its components, its limitations, and how the representation relates to previous topics is skipped or assumed to be covered in a prerequisite course.

As introductory physics is a two semester sequence at FIU, there is a significant mixing of students that occurs at the semester break between MI-Mech and MI-EM. Not all of the students who take the MI-Mech course are able to take the MI-EM (e.g., due to course/work conflicts, major requirements, or class size limitations). Thus, the MI-EM course is comprised of a mixture of students: students who are returning to Modeling Instruction from MI-Mech with a consistent exposure to multiple representations through extensive practice using and talking about the representations in their models and students who are new to the Modeling Instruction environment meaning they most likely took a lecture-based Mechanics course and have not had this kind of experience with representations.

Because the Modeling Instruction environment heavily emphasizes and relies on multiple representations, we are particularly interested in studying how students engage with representations in the Modeling Instruction courses. In this paper, we begin to address this by examining the number and variety of representations that Modeling Instruction students would choose in mechanics and EM contexts, the impact of the second semester (MI-EM) on students' representation choices, and how students' familiarity with the Modeling Instruction class (whether they are new or returning to Modeling in the second semester) impacts their representation use. 


\subsection{Research Questions}

This paper will be addressing three primary research questions: (1) What are the differences between new and returning students' representation choices, particularly in how many and which representations they choose? (2) How do students' representation choices vary based on physics content (mechanics or EM questions)? How does the MI-EM course impact both new and returning students' representation choices over the course of the semester?

\subsection{Methods}

\subsubsection{Problem Solving and Representation Use Survey (PSRUS)}

To answer these research questions, we designed a modified card sort survey about students' choice of representations on various physics problems, which we called the Problem Solving and Representation Use Survey (PSRUS). Rather than asking students to sort representations in person, we created a paper survey that is 25 questions, with 13 questions covering EM topics and 12 questions covering Mechanics topics. In addition to the questions, students were given a list of 15 representations (plus a write-in "Other" option) covered in the Modeling Instruction class. The listed representations (shown in Figure 2) were: Graphs, Equations, Words, Assumptions, Motion Map, Picture, System Schema, Force Diagram, Momentum Vectors, Energy

Pie Charts, Energy Bar Charts, Circuit Diagram, Right Hand Rules (RHR), Potential Lines, and Field Lines. (The entire PSRUS is provided in the Appendix for reference.) For each question, students were asked to choose what representations they would use if they were going to solve that problem. For example, one problem asks "A frisbee is thrown straight up in the air with an initial speed of $3 \mathrm{~m} / \mathrm{s}$. Find the 


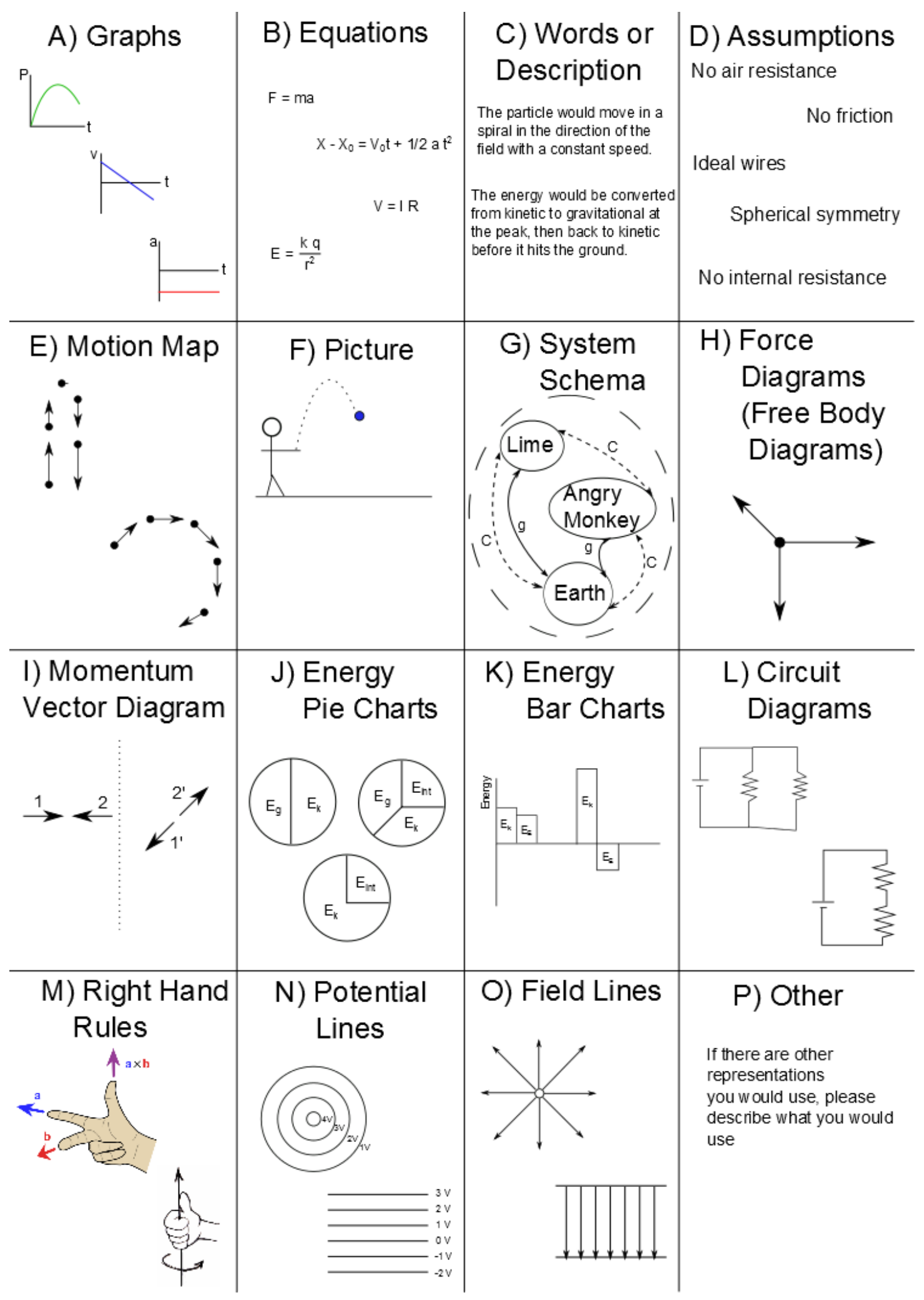

Figure 2: Representations given on the PSRUS.

This figure shows the list of representations that were given to students along with the Problem Solving and Representation Use Survey (PSRUS). 
maximum height the frisbee reaches." Students could respond with any number of the representations that they would use on the problem. For instance, a student may choose equations, a system schema, a picture, and energy pie charts to solve this problem or any other combination of the 16 listed representations. Note that we did not ask students to solve the 25 problems, only to list the representations they would use if they were.

We chose the listed representations for the survey by identifying the representations explicitly covered in the Modeling Instruction courses (both Mechanics and EM) through examination of the curricular materials, instructor's guides, and conversations with MI-Mech and MI-EM instructors about their expectations of what should be contained in a student's solution. In addition, we intentionally included an "Other" option so students could add their own answers to the listed representations. The questions we designed for the PSRUS were meant to cover a broad range of content covered in the Modeling Instruction courses from both Mechanics and EM, with each question open to the possibility of using multiple representations. We also designed many parallel questions between the Mechanics and EM questions (i.e., a "Find the Newton's 3rd Law pairs" question in both Mechanics and EM contexts - Questions 4 and 20 in the PSRUS Appendix). In contrast to most Modeling Instruction problems that ask students to "make a model", we asked students to find a specific quantity in each of the questions so as to not artificially lead students to listing all of the representations.

After the PSRUS was developed, we administered the survey to a test panel, which consisted of faculty, graduate and undergraduate students, and asked for their feedback. In order to address content and face validity in our survey [71], we asked faculty 
and graduate students to judge the appropriateness of the questions for introductory physics students and whether the questions were appropriate for measuring a broad range of students' representation use. Substantive validity, which asks if the intended audience is able to read, respond and engage with the instrument in the intended manner [71], was addressed by administering the survey to undergraduate students and asking for their feedback, particularly on the wording of the questions and instructions and on the time required for survey. In addition, our panel consisted of people who had a wide range of exposure to Modeling Instruction: there were developers of Modeling Instruction, students and instructors who had previously taken or taught Modeling Instruction courses, and students and instructors with no exposure to the Modeling Instruction courses. So this suggests that the PSRUS is interpretable and substantively valid [71] for people who have been in Modeling Instruction before and those who haven't. Furthermore, our test panel was diverse, including representation from: multiple genders, multiple ethnic and racial groups, and bilingual/non-native English speakers. Since the survey was given at a large, Hispanic-serving institution, it was particularly important that the survey be fair and understandable by members of these populations [71].

The feedback from this test panel was used to make substantial edits to the PSRUS. For instance, we cut an "explanation" section from each question due to the length of time required for the survey, and we changed the wording of the instructions to clarify our intent that students were not supposed to solve the problems. In addition, we made changes to wording and questions based largely on the faculty and graduate student feedback about whether the problems were appropriate. 
Finally, we created two forms of the survey, which were given out randomly in the class - one which ordered the EM questions first and one which ordered the Mechanic questions first. When we compared student responses from each version of the test, we found no differences between the two versions for all questions combined, Mechanics questions only, or EM questions only; thus, PSRUS satisfies alternate-form reliability [71]. Further discussion of the validity, reliability, and fairness of the PSRUS is given in the appendices.

Note that the purpose of this survey was to determine what representations students in a Modeling Instruction course thought would be useful in their problem solving and what tools they tended to rely on. For this reason, we did not in any sense try to grade this survey or look for "correct" answers. However, there are some cases that would indicate students not taking the survey seriously, for instance using circuit diagrams on the frisbee problem. In addition, we did not attempt expertnovice comparisons on these data because it has been shown that steps that are useful for experts are not the same as those for novices [19, 20]. Furthermore, it has been pointed out that expert-novice comparisons as a methodology can perpetuate inequity in the field [72].

\subsubsection{Data Collection}

We gave the PSRUS survey pre- and post-instruction to the MI-EM class in the Spring 2015 semester at FIU, which is a large, Hispanic-serving, R1 institution. The survey was administered in class, but there was no grade or extra credit given for completion. A total of 69 students took the pre-survey, 60 students took the postsurvey, and 58 of those students were matched pre-post (which are the data used 
in the following analyses). Of the 58 matched students in the MI-EM course, 30 students were returning from the MI-Mech course and 28 students were new to the Modeling Instruction environment (meaning they took the lecture-based mechanics course at the same institution or had transfer credit for their mechanics physics requirement). These groups of students are referred to as the returning and new students, respectively.

\subsubsection{Data Analysis}

\section{Distributions}

From the survey data, we counted the number of representations that each student chose on all of the questions divided by the total number of representations they could have chosen. We report this as a fraction rather than a pure number because there is a differing number of questions for the mechanics and EM contexts. This gives a single number for each student that represents the average fraction of representations chosen per question. Again, this fraction is the fraction of the total listed representations (the 16 shown in Figure 2), not a measure of "correctness." Based on this fraction, the distribution for a group of students can then be made. An example is shown in Figure 3 showing the distribution for average fraction of representations used on all the questions (both mechanics and EM) in the Pre-PSRUS by all students (both new and returning).

This data set is not normally distributed, with a floor on the left side of the distribution and a right-skewed tail. Based on the format of the PSRUS, this is expected. We expect students to respond with at least one representation on each

question, which accounts for the floor of 0.06 . We would also expect that while most of 


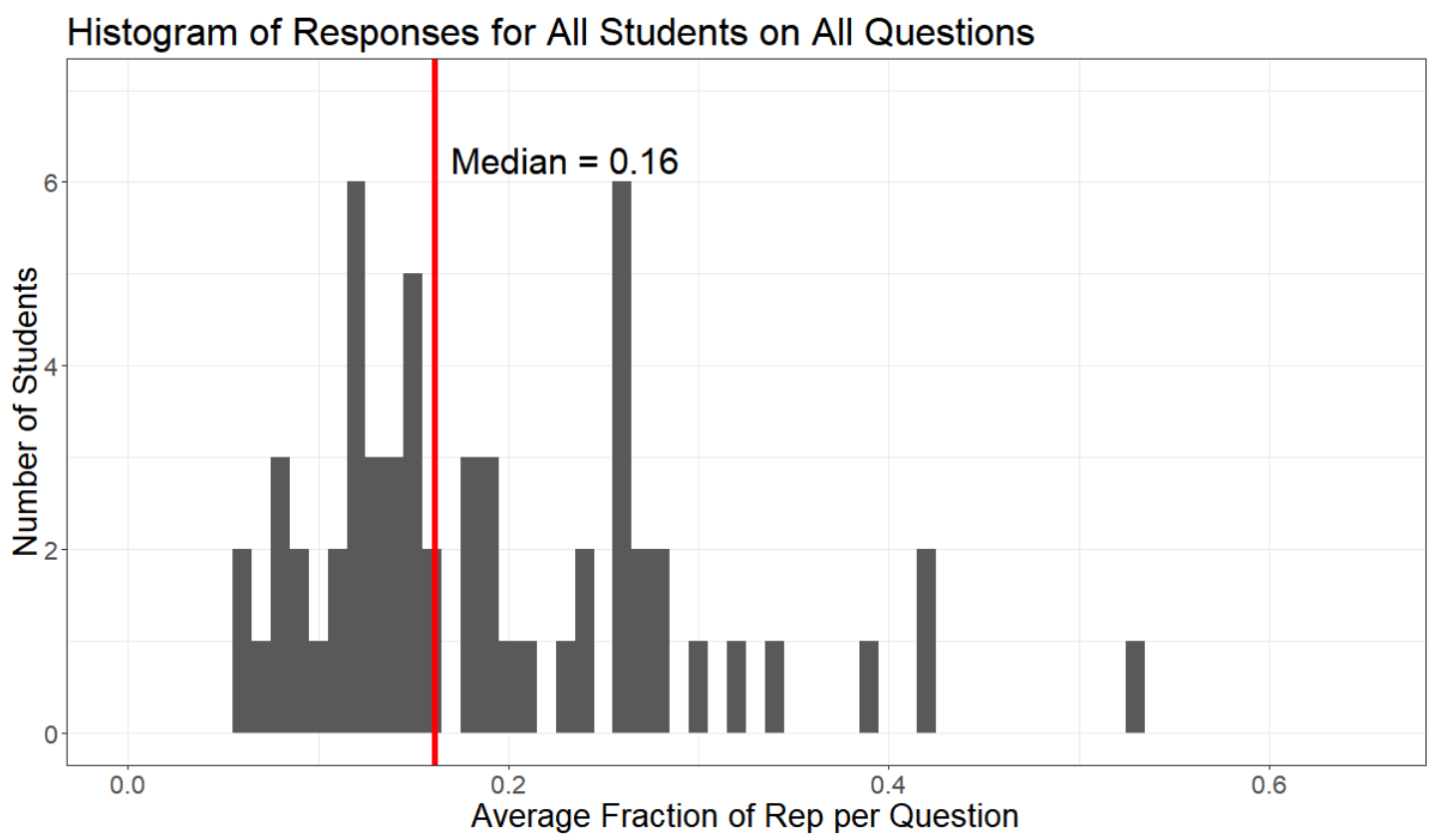

Figure 3: Sample distribution of PSRUS responses.

This distribution shows the average fraction of representations chosen on all questions combined by all the students in the MI-EM class on the Pre-PSRUS. The median is marked at 0.16 or between 2-3 representations per question.

the questions lend themselves to multiple representation, not all of the representations are applicable to every question, thus accounting for the right-skew tail. This type of distribution has implications for the statistical tests and averages that we report. Since the distribution is not (and would not be expected to be) normal, we used non-parametric statistical tests and effect sizes, which are expanded upon in following sections. We also report median values as the measure-of-center for these distributions because the mean is more drastically affected by the right-skewed tail.

In a similar manner, we created distributions that were separated by student group (returning or new) and by content area (Mechanics, EM, or All) for both the preand post-PSRUS data. As expected, all of these distributions held the same shape as that shown in Figure 3: non-normal, left floor, and right-skewed tail. 


\section{Wilcoxon-Signed-Rank and Wilcoxon-Mann-Whitney tests}

To compare these nonparametric distributions, we used Wilcoxon-Signed-Rank (WSR) tests and Wilcoxon-Mann-Whitney (WMW) tests, which are the respective nonparametric equivalents to paired and unpaired t-tests that require no assumptions about the shape of distribution. We used paired WSR comparisons for all pre vs post and Mech vs EM comparisons since the same students comprised these populations, whereas unpaired WMW was used for all new vs returning comparisons as these are independent populations. Both WSR and WMW tests are based on a rank-sum procedure and thus are able to distinguish between shape and placement differences of distributions; however, when the distributions have the same shape, the WMW and WSR tests can be interpreted as a difference in medians [73, 74, 75]. Since all of our distributions have the same general shape, we will interpret differences by the WSR and WMW tests as differences in the medians of the distributions.

Given that we ran multiple comparisons (9 pre vs post, 6 new vs returning, 6 Mech vs EM), we used an alpha level for each test of 0.00238 , based on the Bonferroni Correction of:

$$
\alpha_{B 1}=\frac{0.05}{21}=0.00238
$$

which gives a 95\% confidence in the overall study based on 21 individual tests. The Bonferroni correction is often cited as the most conservative correction for multiple tests [76] ; however, it is the best correction for both family-wise error rates and per-family error rates on Type 1 Error [77]. 


\section{Cliff's Delta}

To compare individual representations (rather than an average fraction as was done in the WSR and WMW tests), we used Cliff's Delta as a nonparametric effect size measure, which makes no assumptions about the shape of the distributions. Cliff's Delta is calculated from comparing the number of data points from Distribution X that are larger than the number of data points from Distribution Y divided by number of data points in each distribution as shown in Equation 2, with a correction if the distributions are paired [78].

$$
\delta=\frac{\#\left(X_{i}>Y_{j}\right)-\#\left(X_{i}<Y_{j}\right)}{n_{x} * n_{y}}
$$

In contrast to Cohen's $d$ which has no upper limit, the Delta effect size is limited to be between -1 and 1 . Cohen's interpretation of small $(d=0.2)$, medium $(d=0.5)$ and large $(d=0.8)$ effects [79] then correspond to Deltas of $\delta=0.147, \delta=0.33$, and $\delta=0.474$ respectively $[80]$.

Since we used Cliff's Delta to compare the 16 representations, which each had their own effect size, we again used a Bonferroni correction to calculate the confidence intervals for each effect as shown in Equation 3.

$$
\alpha_{B 2}=\frac{0.05}{16}=0.003125
$$

Thus, the reported confidence intervals on each effect size are $99.7 \%$ confidence intervals so that the overall comparison of the set of 16 representations has a confidence of $95 \%$. 


\section{Boxplot Comparisons}

In cases where the Cliff's Delta comparisons are not meaningful or there is complete overlap in confidence intervals, we use box plots to compare differences in the 16 representations. The box plots are able to show differences not only in median but also in the shape of the distribution, which is lost information in the effect size analysis. However, in using box plots, we lose the statistical significance of the comparison. While we could use WMW tests to compare the 16 representations, the shape of the distributions are no longer the same when comparing each representation (rather than all representations combined). This means that a significant difference by the WMW could be due to a difference in the shape of the distributions or a difference in the placement of the distributions, with no way to tell which is the significant factor. Thus, we opt to present box plots without statistical significance in these cases because it best presents the information of interest, but these should not be used as an extensive, generalizable comparison.

\subsection{Results and Discussion}

A summary of the average representation choice per question by content and by group of students is shown in Figure 4 for the pre- and post-PSRUS. The average percentage of representations per question chosen ranges from $13.4 \%$ to $30.5 \%$, or somewhere between 2-5 representations per question. This indicates that students are neither selecting only one representation repeatedly nor selecting all the representations consistently, but are being thoughtful in their representation choices. The features and conclusions from this plot will be discussed in the subsequent sections. 


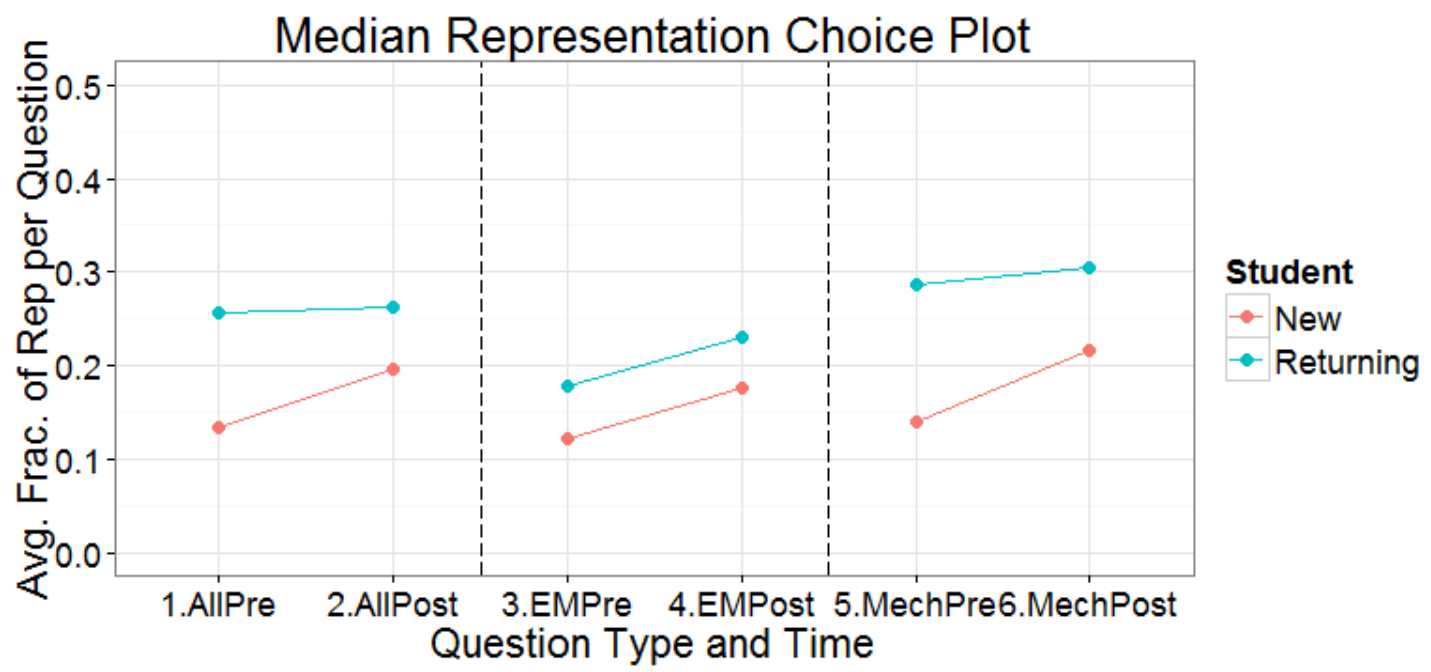

Figure 4: Summary of medians from PSRUS results.

This plot shows the median fraction of representations that students chose per question separated by content area and group of students.

\subsubsection{Baseline - New vs returning}

When comparing new vs returning students, we see significant differences $\left(p<\alpha_{B 1}\right.$ by WMW) between students' representation choices on AllPre, MechPre, and MechPost (all other new vs returning comparisons are not significant - shown in Table 3 in Appendix), which is consistent with our previous results [81]. This tells us that students are entering the MI-EM course making different representation choices, primarily in Mechanics content. While this is not entirely surprising given the differences in prior instruction and representation emphasis, the Mechanics Pre comparison in particular provides a good baseline for differences between representations in Modeling Instruction and traditional lecture courses.

As is shown in Figure 4 Column 5, returning students on average use $28.7 \%$ of the available representations per question. This means that returning students are saying they would use 4-5 representations on each Mechanics question. In contrast, 


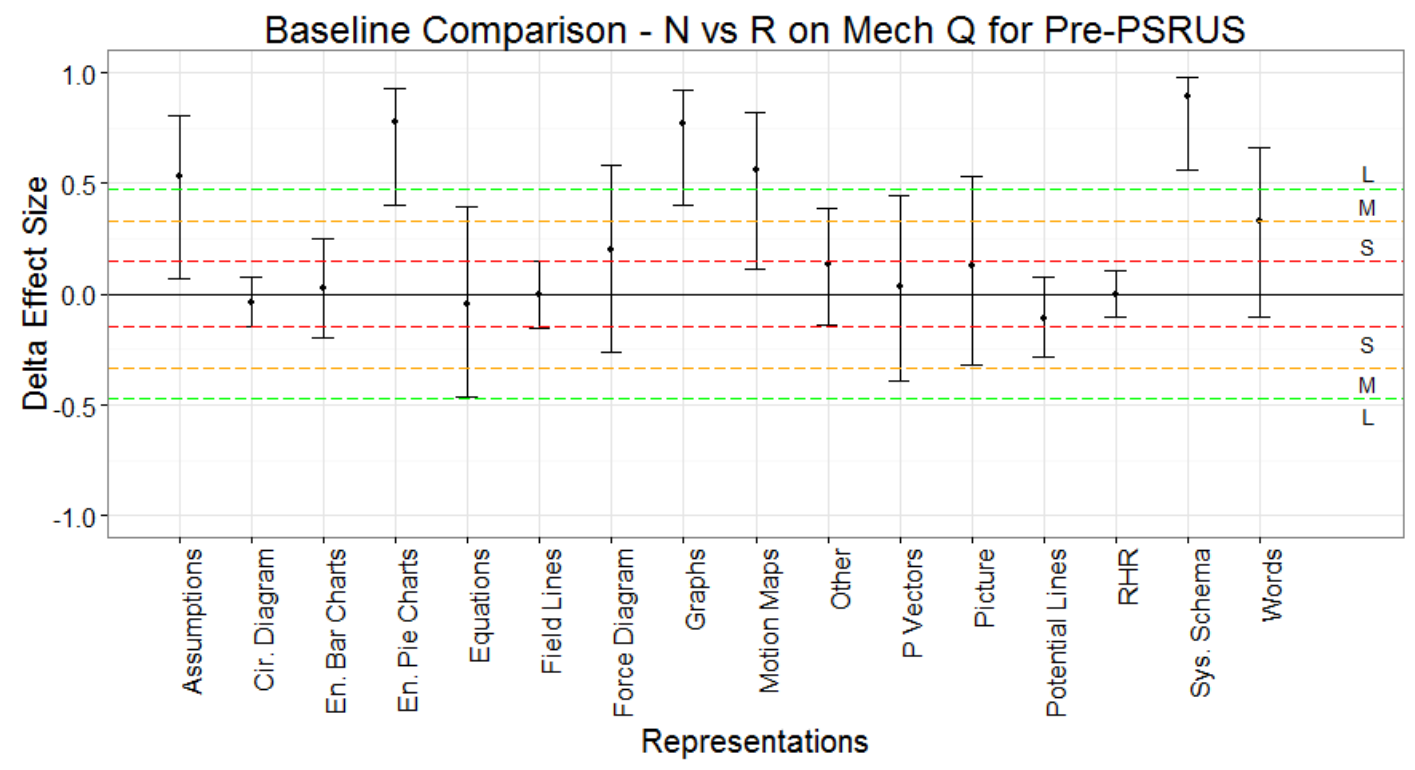

Figure 5: Cliff's Delta effect sizes comparing new and returning students. Cliff's Delta effect size for each representation comparing new and returning students on Mechanics Questions from the Pre-PSRUS with $99.7 \%$ confidence intervals (from $\left.\alpha_{B 2}\right)$. Positive effects show a representation that is favored by returning students; negative effects show those favored by new students.

new students say they would use $14.1 \%$ of the available representations on average, which is between 2-3 representations.

When we break it down by the individual representations, we see more differences between new and returning students. The Cliff's Delta effect size for each representation on Mechanics questions from the Pre-PSRUS is shown in Figure 5. In this comparison, a positive effect means that the representation is favored by returning students and a negative effect means that the representation is favored by new students.

From the effect sizes, we see that there are several large positive effects, meaning there are several representations that are highly favored by returning students over new students. These include some representations that we would expect to be fa- 
vored by returning students, such as Energy Pie Charts [13] and System Schema [8], which were developed for and almost exclusively used in Modeling Instruction courses. However, there are also several representations including Assumptions, Graphs, and Motion Maps that we might expect to be universal (used by both lecture and Modeling Instruction course) that we see are favored by returning students. This means that, as a baseline, returning students use more representations because not only do they know more representations, but they see more traditional representations as more widely applicable.

We also see no difference between new and returning students' use of equations or force diagrams in pre data. In the MI-Mech curriculum, two of the largest modifications from the typical mechanics content organization are that 1) the MI-Mech curriculum introduces energy before forces and 2) there is a large focus in the course materials on conceptual understanding and use of representations before introducing equations [26]. The fact that we see no difference between new and returning students on equations and force diagrams indicates that these changes to the MI-Mech curriculum are not detrimental to students' understanding of when these representations are appropriate.

\subsubsection{Returning Students}

When we separate students' responses by whether they were new or returning to Modeling Instruction, we see two distinct patterns in their representation choices. Starting with returning students, we see a high level of representation use that is maintained across the pre- to post-PSRUS in MI-EM, which is particularly evident in Columns 1-2 (All questions combined) in Figure 4 where there is virtually no change 


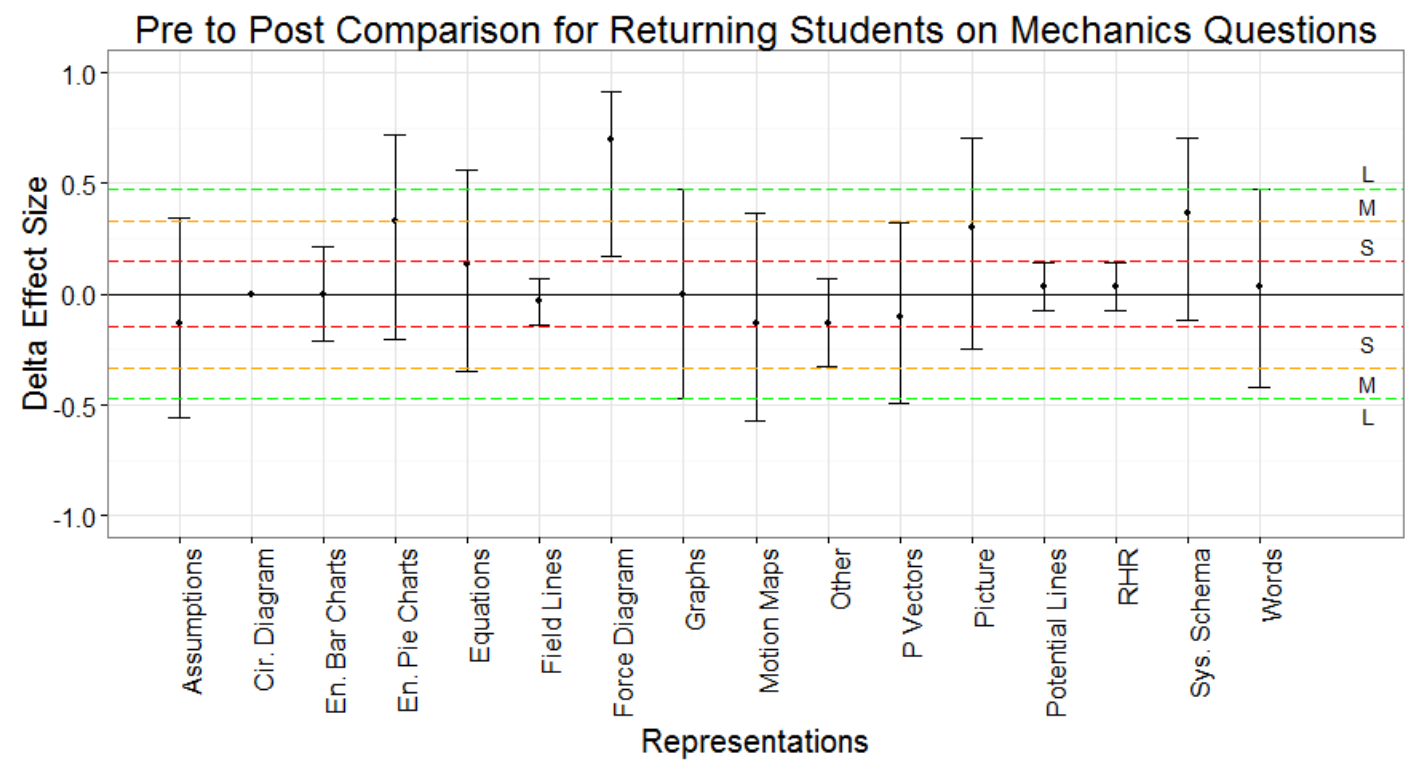

Figure 6: Cliff's Delta effect sizes comparing pre- to post-responses. Cliff's Delta effect size for each representation comparing pre to post representation choices on Mechanics Questions from returning students with 99.7\% confidence intervals (from $\alpha_{B 2}$ ). Positive effects show a representation that is favored in the post-PSRUS; negative effects show those favored in the pre-PSRUS.

in the average number of representations that students choose per question. When we split the responses by content area, we do see an increase from pre to post in both EM (17.8\%-23.1\%) and Mechanics (28.7\%-30.5\%) questions, but neither of these shifts are significant at the $\alpha_{B 1}$ level by WSR tests (See Table 1 in Appendix for reference).

When examining the individual representations, we see similar results. The effect sizes for the representations chosen on Mechanics questions are shown in Figure 6. In the comparison of pre to post, a positive effect size means the representation was chosen more often in the post-survey whereas a negative effect size means the representation was chosen more often in the pre survey.

From Figure 6, we see that most of the representations have small or negligible effects, meaning there were no changes from pre to post in the use of these represen- 
tations. We see medium, positive effects in Energy Pie Charts, Pictures, and System Schema, which suggests that MI-EM may help students see the applicability of these representations in Mechanics content; however, we do not have the statistical power to conclusively say so. The one representation that is significant at the $\alpha_{B 2}$ level is Force Diagrams, which has a large, positive effect. This tells us that over the second semester of Modeling Instruction, students are increasing their use of Force Diagrams on content that wasn't introduced that semester (although force diagrams were used).

The results of Figure 6 are important because they tell us that returning students are maintaining, if not increasing, their representations on content from the previous semester.

Likewise, the effect sizes of returning students' representation choices on EM questions are shown in Figure 7. Again, a positive effect means the representation was favored in the post-PSRUS, and a negative effect means the representation was favored in the pre-PSRUS. As before, many of the representations have negligible or small effects. The only representation with a significant effect at $\alpha_{B 2}$ is the Right Hand Rule, which is a huge, positive effect. Energy Bar Charts, Energy Pie Charts, Force Diagrams, and System Schema have medium, positive effects, which suggests that students have increased their use of these representations in the post-PSRUS; however, their confidence intervals are large enough to cross zero. Interestingly, Circuit Diagrams has a medium, negative effect (though not significant), which suggests that students thought that Circuit Diagrams would be more useful at the beginning of the semester than they did at the end.

Figure 7 tells us that while there is some growth in returning students' use of representations on EM problems, they are maintaining the number of representa- 


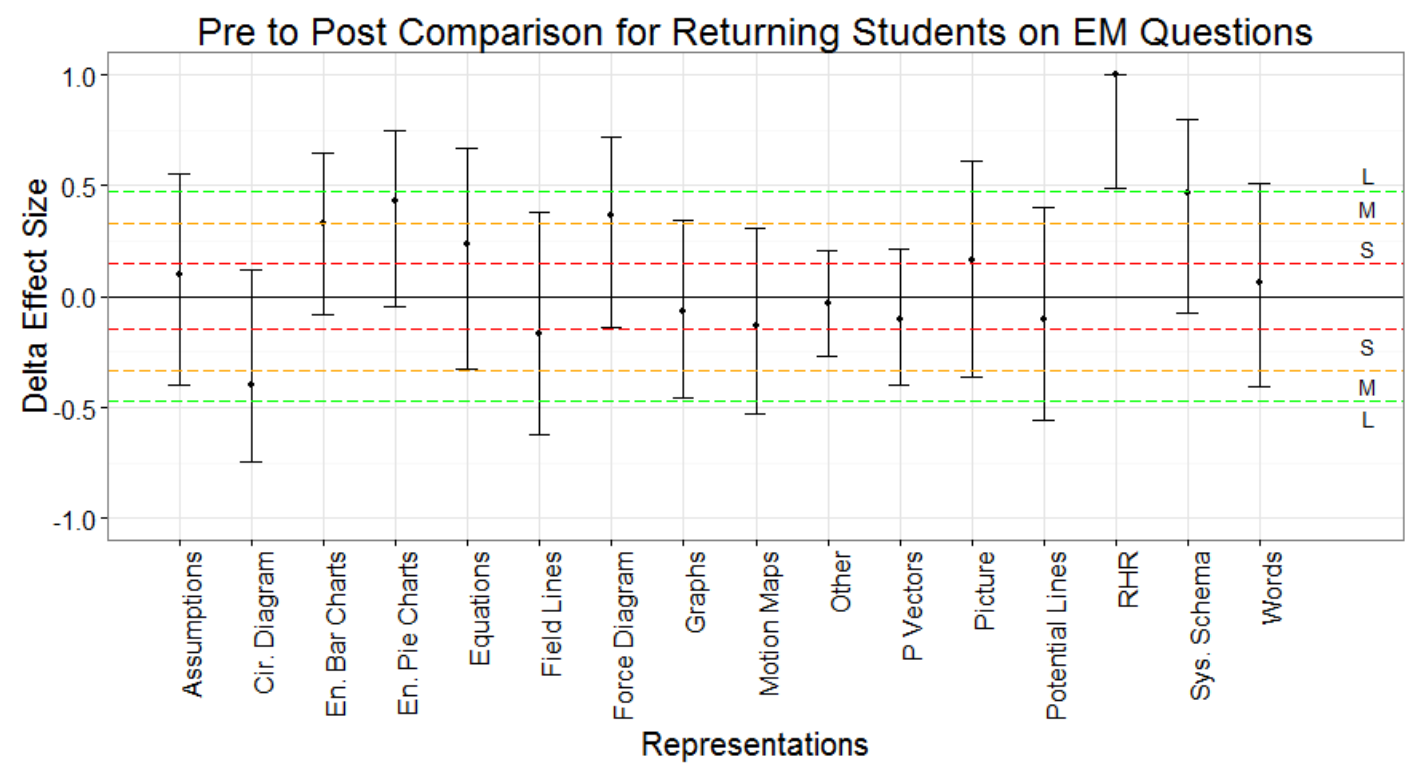

Figure 7: Cliff's Delta effect sizes comparing pre- to post-EM responses.

Cliff's Delta effect size for each representation comparing pre to post representation choices on EM Questions from returning students with 99.7\% confidence intervals (from $\alpha_{B 2}$ ). Positive effects show a representation that is favored in the post-PSRUS; negative effects show those favored in the pre-PSRUS. 
tions from pre to post. This could indicate that returning students are approaching new material with a "try all representations" strategy, even in unfamiliar contexts. However, while maintaining representation use is good on both Mechanics and EM problems, it is concerning that we do not see more growth of representations in EM, particularly in Field Lines and Potential Lines, which are two of the new representations introduced in MI-EM. This result may indicate that more scaffolding is needed around these representations in the curriculum.

Furthermore, we see large differences between the number of representations that returning students use on Mechanics questions compared to EM questions. On the pre-survey, returning students choose on average $17.8 \%$ of the representations per question for EM and $28.7 \%$ for Mechanics, which is a significant difference by WSR test at $\alpha_{B 1}$. This kind of difference at the beginning of the semester is not shocking, but the difference is persistent to the end of the MI-EM semester. Even on the postsurvey, returning students choose significantly fewer (by WSR at $\alpha_{B 1}$ ) representations on EM questions (23.1\%) than on Mechanics questions (30.5\%). (Shown in Table 2 in Appendix for reference.)

When we compare the individual representations on Mechanics and EM questions, there are clear distinctions between what returning students see as useful on Mechanics problems and what they see as useful on EM problems. Figure 8 shows the effect size analysis for the returning students on the post-PSRUS, looking at differences in representation use between Mechanics and EM questions. In this comparison, a positive effect means the representation was favored on EM questions, and a negative effect means it was favored on Mechanics questions. 


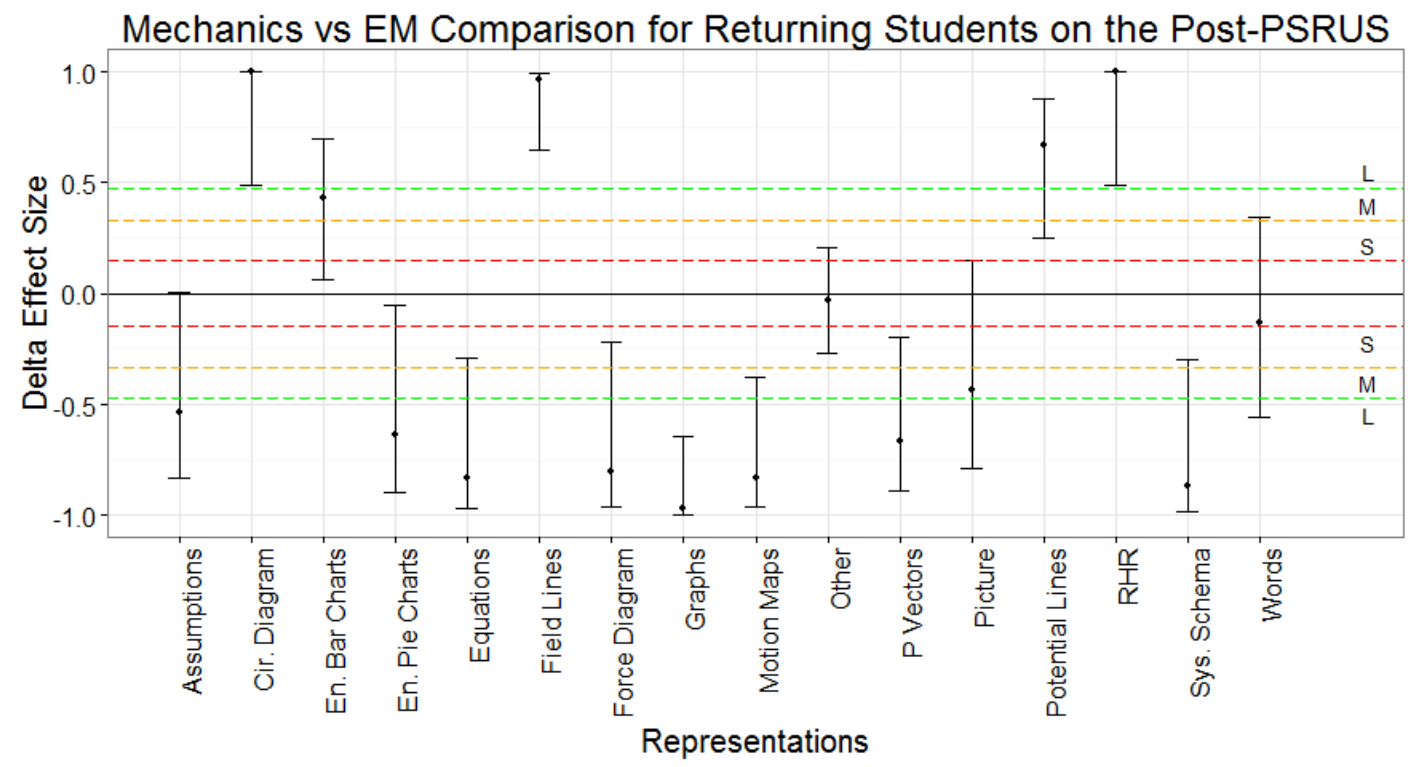

Figure 8: Cliff's Delta effect sizes comparing mechanics to EM.

Cliff's Delta effect size for each representation comparing Mechanics to EM representation choices on the post-PSRUS from returning students with $99.7 \%$ confidence intervals (from $\alpha_{B 2}$ ). Positive effects show a representation that is favored on EM questions; negative effects show those favored on Mechanics questions. 
As would be expected, the representations that are favored on EM questions (large, significant, positive effects) are those that were introduced in the MI-EM course: circuit diagrams, energy bar charts, field lines, potential lines, and right hand rules. Words, Pictures and Assumptions have confidence intervals that cross zero, thus cannot be categorized as either Mechanics or EM (though we do recognize that both Assumptions and Pictures have large, negative effect sizes). With the exception of the "Other" category, the rest of the representations were highly favored on Mechanics questions as evidenced by the large, significant, negative effects.

While some representations are certainly more useful in EM or Mechanics contexts (i.e., circuit diagrams are generally exclusive to EM contexts), there are many representations that we wouldn't expect to be so polarized. For example, Equations, Graphs, and Force Diagrams have some of largest negative effects, but from an expert perspective are certainly useful tools in EM. Any introductory textbook in EM will present Maxwell's equations as the foundation of EM, will show Force Diagrams with example problems involving the electric or magnetic force and will use electric field vs position or magnetic flux vs time graphs to highlight the relevant relationships $[46,45]$. However, we see that the returning students in MI-EM are choosing these representations more heavily in Mechanics, which may indicate that they are not seeing the applicability of these representations in the new EM contexts.

\subsubsection{New Students}

In contrast to the returning students, the new students in MI-EM do see significant growth in the number of representations that they use over the course of the semester. For all questions combined, new students, on average, increase their representation 
choices per question from $13.4 \%$ to $19.6 \%$, which amounts to a gain of one representation per question. This increase is shown from Column 1 to 2 in Figure 4 . When we split the questions by content, we see very similar patterns. EM representations increase from $12.3 \%$ to $17.6 \%$ and Mechanics representations increase from $14.1 \%$ to 21.6\% (shown in Columns 3-4 and 5-6 in Figure 4, respectively). All of these gains are significant by WSR at $\alpha_{B 1}$ (see Table 1 in the Appendix for reference).

In itself, this is an important result. In addition to impacting students' use of representations in the EM content that is taught in the class, new students in MIEM are increasing and backtracking representations to physics content that was not explicitly covered in the semester. This indicates that Modeling Instruction is not only teaching conceptual physics knowledge but is influencing the tools that students use to problem solve regardless of content. Furthermore, we see relatively even gains in representations between Mechanics and EM questions for new students, unlike the returning students who favor representations in Mechanics. By the WSR tests, there were no significant differences between EM and Mechanics in either the pre- or post-PSRUS.

When we examine the individual representations that students chose on EM questions, we see that the new students are both gaining the new representations emphasized in MI-EM course and using familiar representations more frequently. The box plots in Figure 9 summarize the changes for new students in each representation from pre- to post-semester. Overall, we see increases in the representations introduced in MI-EM, particularly in RHR and Field Lines as evidenced by the large jump not only in the median but also in the inner quartile range (IQR). We also see that the representations that are unique to Modeling Instruction (Energy Pie Charts and System 
Schema) are used more frequently in the post. There is a large change in the spread of the IRQ for Energy Pie Charts and System Schema, accompanied by a increase in the median value. Beyond that we see large shifts in the spread of the distributions for the more familiar representations as evidenced by a larger IRQ in the post-data; this says that by the end of the semester new students are using words, assumptions, pictures, and force diagrams more frequently on EM problems.

By looking at how new students respond to the Mechanics questions, we can see what representations students are backtracking and transferring out of the class content. These results are summarized in the box plots shown in Figure 10. We do see new students using representations that they would have learned in the MI-EM course on Mechanics questions - primarily the System Schema, which shows a large spread in the distribution and an increase in the median for the post-survey. However, most of the large increases in representations on the Mechanics questions are coming from those that they are already familiar with. There are large overall shifts in both the median and IQR to the distributions of Pictures, Force Diagrams, and Assumptions as well as changes to the shape of the distributions in Graphs, Words, and Momentum Vectors as evidenced by the larger IQR's.

This suggests that MI-EM is helping new students recognize the applicability of the representations that they already know, which they are then able to apply to the new EM context as well as backtracking to the more familiar Mechanics context.

\subsection{Conclusions}

To answer Research Question (1), we compared new and returning students' representation choices on the PSRUS Mechanics and EM questions. We found a sig- 

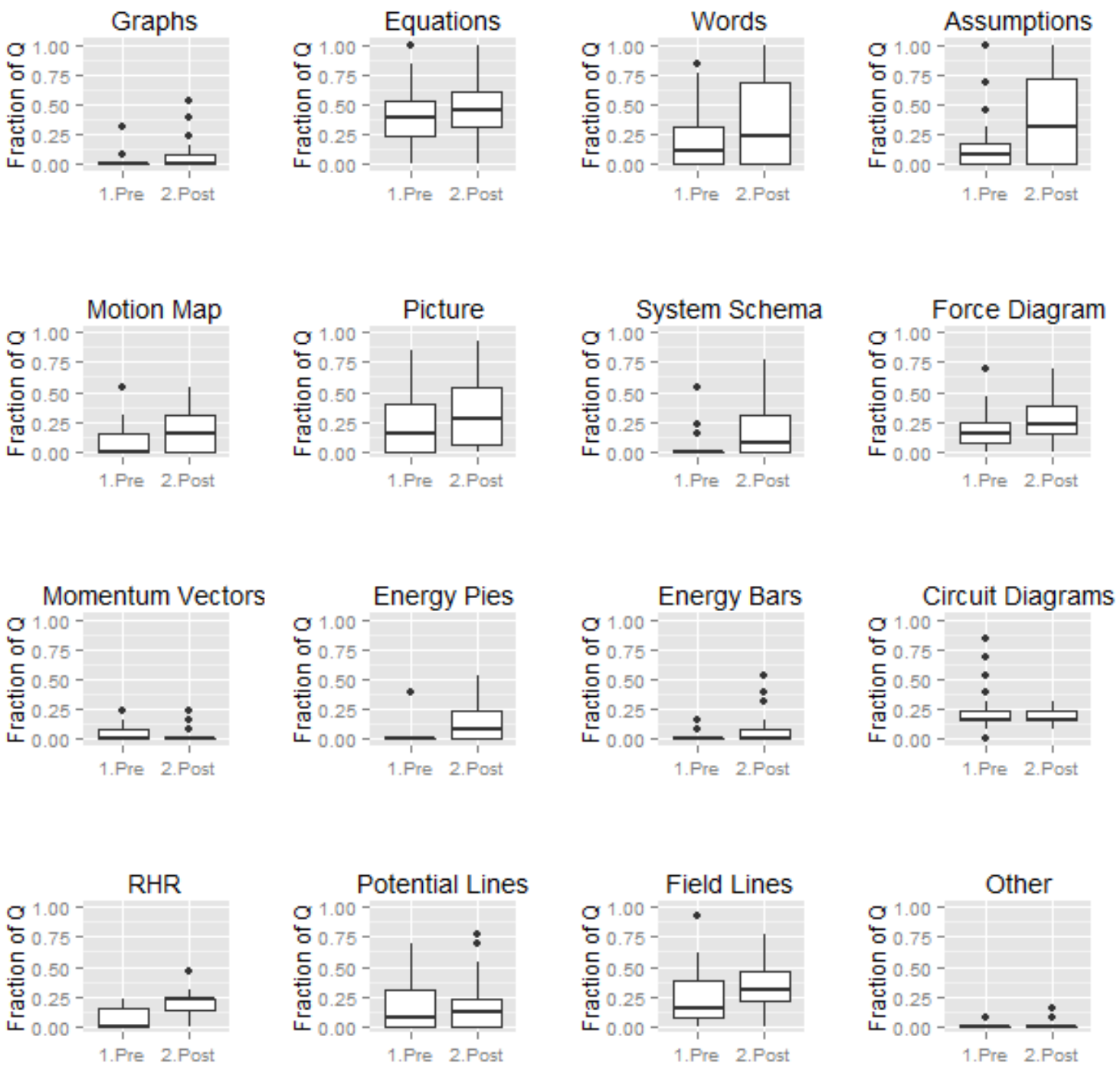

Figure 9: Box plots comparing pre to post on EM questions for new students. These box plots show the side-by-side comparison for the frequency of each representation in the pre- and post-PSRUS on EM questions for new students. The number of questions that students chose that representation is presented as a fraction of all EM questions on the vertical axis. 

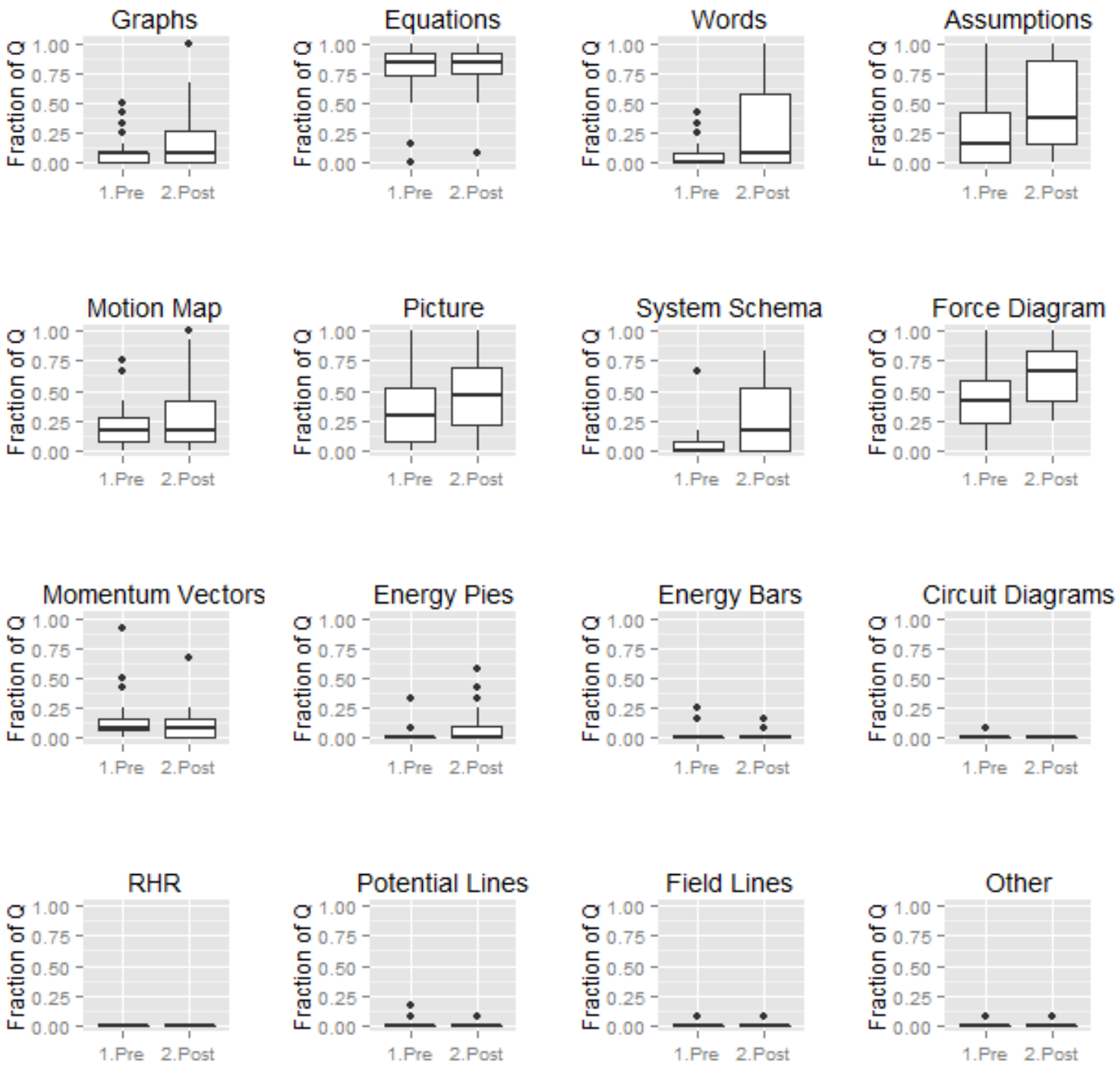

Figure 10: Box plots comparing pre to post on mechanics questions for new students. These box plots show the side-by-side comparison for the frequency of each representation in the pre- and post-PSRUS on Mechanics questions for new students. The number of questions that students chose that representation is presented as a fraction of all Mechanics questions on the vertical axis. 
nificant difference on the mechanics pre-survey questions that was persistent to the post-survey. In particular, the difference between new and returning students on the mechanics pre-survey is a good baseline for comparing how Modeling Instruction and traditional lecture courses teach representations. From the Cliff's Delta analysis (in Figure 5), we showed that not only do returning students use more novel tools like energy pie charts and system schema on mechanics questions, but they also use familiar representations like graphs, motion maps, and assumptions more frequently. We also see no differences in students' use of equations or force diagrams, despite a qualitative-first, energy-first curriculum. This result is consistent with Traxler and De Leone and Gire who found that students from inquiry based physics classes use more representations than their lecture counterparts $[67,49]$; however, we have moved beyond their results by looking at which representations Modeling Instruction students use more and in which contexts.

To answer Research Question (2) and (3), we looked at how new and returning students' representation choices changed from pre to post. We found that returning students maintain high levels of representation use, with the only significant growth of representations in right hand rules for EM questions and force diagrams for mechanics questions. This suggests that returning students have a high retention of representations and are not changing their views of when the representations are applicable. However, we do not see significant growth in representations on EM questions for returning students even though MI-EM curriculum introduces five new representations and builds on those from Mechanics, nor do we see returning students reaching similar frequency of representation use that they do on Mechanics questions (addressing Research Question 3). While we would not expect exactly the same representation 
use in Mechanics and EM contexts, the fact that returning students heavily favor general representations like equations, graphs, and pictures in mechanics contexts is concerning (addressing Research Question 2), which could indicate that the MI-EM curriculum is not integrating and emphasizing the representations in a consistent manner to the MI-Mech course.

In contrast, the new students in MI-EM see significant growth in their representation choices from the beginning to the end of the semester (addressing Research Question 3), regardless of Mechanics or EM content (addressing Research Question 2). The fact that the new students see even growth between the content areas further supports the idea that there are differences in how representations are taught between MI-Mech and MI-EM. Even more importantly, we are seeing that the new students in MI-EM are backtracking what they have learned about representations to physics content that was not taught in the course. This suggest that MI-EM is impacting students' problem solving skills in addition to their content knowledge and providing them with more tools to use in that process. Furthermore we see that the gains in representations come from both gaining new representations (i.e., energy pie charts and system schema) and using familiar representations more frequently (i.e., words, force diagrams, pictures) from Figures 9 and 10. That being said, the new students never reach the level of representation use that the returning students have. On mechanics questions, there was a persistent, significant difference between number of representations that the new and returning students chose. On EM questions, we see that the number of representations the new students end with is about the same as the number of representations that the returning students start with. This indicates 
that the MI-EM curriculum is not doing a good job of catching the new students up to speed with the returning students.

These results suggest that MI-EM is meeting its goal of increasing the number and variety of representations that they use in physics. Its students have access to more representations and use more traditional representations in physics more frequently. This result is consistent with what other researchers have found for reformed, inquiry based physics courses $[49,67]$. Regardless of the content being taught, Modeling Instruction is impacting how students problem solve and the tools that they rely on to do so. However, we also see significant differences between new and returning students' representation choices, which indicates that these two groups of students are having different experiences in the class. From these results, curricular changes to MI-EM are indicated, particularly to incorporate representations consistently across the two semesters and to transition the new students into Modeling Instruction more effectively.

This study also has wider implications for physics instructors and researchers. Particularly for reformed classes, we need to pay attention to how much we are building on students' experiences in previous courses. This is especially true of the second course in the sequence, like MI-EM, which may require more introductory scaffolding than is given. Furthermore, this study exemplifies a course evaluation based on a non-content related learning goal. While multiple representation use is a core tenant of Modeling Instruction, previous measures of success would not have been able to address whether students found these representations applicable. We encourage instructors and curriculum developers to assess their classes on learning goals that move beyond content knowledge. 


\section{EXTENDING UNIVERSITY MODELING INSTRUCTION TO INTRODUCTORY ELECTRICITY AND MAGNETISM}

\subsection{Abstract}

Modeling Instruction is an introductory, calculus-based university physics curriculum that is centered around the development, validation, use and revision of conceptual models in physics through guided-inquiry activities. For a variety of phenomena presented in class, students work in small groups of 3 students and larger discussion groups ( 24 students) to develop conceptual models, which consist of multiple, coordinated, purposeful representations (i.e., equations, force diagrams, graphs, pictures, etc.). As the foundation of the model, representation use and coordination is a central tenant of the Modeling Instruction curriculum and is the focus of the first two steps in the modeling cycle outlined by Brewe. The first semester of Modeling Instruction Mechanics (MI-Mech) has been developed at multiple institutions across the United States over the past 15 years and has yielded significant gains in students FCI scores, affective measures, and student networks in the classroom when compared to traditional lecture-based courses. In the present paper, we will present the work done to extend the success of the Modeling Instruction framework to the second introductory physics course - Electricity and Magnetism (MI-EM), including the ways in which the Modeling Theory of Science, the Modeling Theory of Instruction, and the Modeling Discourse Management were instantiated in the course. We will also present our preliminary evaluation of the MI-EM curricular changes from a survey of students' representation choices across the introductory physics content. Since representation use is such a central learning goal of the class, we were particularly interested in how the curricular changes impacted students representation choices. 
Using Mann-Whitney-Wilcoxon tests, Wilcoxon-Signed-Rank tests, and Cliffs Delta effect sizes, we compare data from before and after the curricular changes, where we see significant gains particularly in the number and variety of representations that students choose.

\subsection{Introduction}

Modeling (i.e., the process of creating and using models) is practiced widely throughout physics, from introductory classrooms to advanced scientific theories in conceptual development to experimental laboratories [82, 26, 29, 28, 83, 84]. Since the process of modeling is critical to the practice of physicists (and scientists more generally) $[1,85]$, there have been many efforts to incorporate models and modeling into physics courses $[26,86,84,87,88]$.

University Modeling Instruction (MI) is one such physics curriculum, which has been intentionally designed around the creation and use of models in introductory, calculus-based physics. Within MI courses and MI theoretical framework, a model is defined as a purposeful, coordinated set of representations of a particular class of phenomena that exists in the shared domain discourse and is an inscription of disciplinary knowledge $[2,27]$. In practice, this model definition means that MI activities are designed to guide students in developing, testing, applying, and refining a shared conceptual model (in contrast to an individual mental model) consisting of multiple representations that can be applied to set of phenomena. For example, the first 10 weeks of the MI course for introductory mechanics (MI-Mech) are aimed at helping students create and use the constant acceleration model in a variety of contexts. When asked to create a constant acceleration model for any particular phenomenon (i.e., a 
stone being thrown upwards) at the end of those 10 weeks, we would expect MI-Mech students to create a set of representations that fully describe what happens to the stone, including graphs, equations, pictures, a free body diagram, a word description, energy pie charts, a motion map, a system schema, and an explicit statement about the assumptions that students are making in their model. These representations (or the tools that students use when problem solving) should be coordinated and telling a similar story; for example, as the speed of the stone would decrease, this should be reflected in a negative slope on the velocity vs time graph, in a decreasing amount of kinetic energy in the energy pie charts, and in a decreasing size of arrow in the motion map.

The mechanics course of University Modeling Instruction (MI-Mech) was developed at multiple institutions building off of the high school Modeling Instruction materials [36]. Over the last decade, there have been multiple research projects conducted within the MI-Mech course at Florida International University (FIU), which have shown that the MI-Mech course achieves larger conceptual gains on the Force Concept Inventory [39] when compared to similar lecture courses and MI-Mech students are more likely to pass the course (i.e., a reduction in the fail-drop-withdraw rate) [38]. In addition, MI-Mech course has shown that students have more favorable attitudes towards physics (as measured by the CLASS survey [42]) by the end of the semester, independent of instructor [41]; in contrast, most students in physics courses in the U.S. see a decrease in favorable attitudes $[42,43]$. Students in MI-Mech also have increased participation over the course and form a network of students that they work with when compared to lecture, which contributes to their success in the course $[89,44]$. In a longitudinal study of physics majors, physics students who took 
MI-Mech are just as likely to reach graduation as their mechanics lecture counterparts, with women actually being more likely to graduate with a physics degree than men [90]. Given the success of the MI-Mech course along multiple measures, we have worked over the past three years to extend the MI framework to the second semester introductory physics course - Modeling Instruction Electricity and Magnetism (MIEM), which we present in the sections below.

While we continued to use multiple measures to assess the MI-EM course, these measures (including conceptual surveys and physics identity measurements) did not necessarily align with the primary goal for the course, which was building and using models. Moreover, with ample evidence that the MI-Mech course leads to better outcomes than lecture-based alternatives, we were more interested in comparing the MI-EM course to a course in which students construct knowledge and interact, but without the strong emphasis on coherent models (as opposed to making a straw man comparison to a lecture course)[91]. In order to examine what parts of modeling students are finding useful, we decided to focus our assessment (for course evaluation and research purposes) particularly on the choices that students make about the representations that they would include in their model. As representations play a critical role in how and what students are creating in the modeling process, we present the following representation analysis as a means of examining students' modeling skills.

Thus, the purpose of this paper is two-fold: 1) to articulate and highlight how the Modeling Instruction framework was applied and instantiated into a university, calculus-based introductory electricity and magnetism course and 2) to assess the MIEM curriculum by examining students' representation choices before and after the 
curricular changes. To that end, we first present a literature review of the Modeling Instruction framework and the role of representations in the teaching and learning of physics. Then, we will describe the specific modifications we made to the MI-EM course and how those relate to the theoretical framework. Following that, we will discuss the survey we developed, the data collection and analysis methods, and the conclusions we draw from the results of the survey.

\subsection{Literature Review}

In the following sections, we outline the theoretical framework that motivated the development of the Modeling Instruction courses and influenced the design of the classroom structures. We also present the research on multiple representation use in physics and science more generally.

\subsubsection{Modeling Instruction Theoretical Framework}

University Modeling Instruction (MI) is a reformed, research-based introductory physics class that was particularly designed for university level, calculus-based physics classes [37]. At the center of the MI course, there are two integrated theories that motivate the design of the class: the Modeling Theory of Science [28, 32] and the Modeling Theory of Instruction $[32,26]$. Additionally, there is a set of strategies for managing classroom interactions, Modeling Discourse Management [34, 35]. For the purpose of illustrating each of these theories and their instantiation in the classroom, we present examples from the MI-Mech course. (In subsequent sections, we will describe their instantiation in the MI-EM course.) 


\section{Modeling Theory of Science}

Modeling Theory of Science (MTS) is developed from the idea that science is the process of developing, testing, applying, and refining models. This process is what it means to be "doing science" and that as practicing scientists, we engage in the MTS process on a daily basis $[29,28,1]$. It is important to note that a model in this context is not simply a small-scale model, a physical replication, or an individual mental model. Instead, we are referring to an abstract, conceptual model that serves as a mediating structure between individual concepts and large physical theories [32]. Within the MI framework, we specifically define a model to be a purposeful, coordinated set of representations of a particular class of phenomena that exists in the shared domain discourse and is an inscription of disciplinary knowledge $[27,2]$. Instructionally, conceptual models are built and used to understand a certain class of phenomena by explicitly describing the salient features, predicting the outcomes, and explaining why an event transpires.

Modeling, as a practice, is used widely throughout physics, from the introductory level to the advanced theories. For example, Nercessian describes how Maxwell employed models in constructing the electromagnetic field and how he used "model-based reasoning" to extend familiar representations of the field and to generate new representations that ultimately provided the insight for what are now known as Maxwell's Equations [29]. At the introductory physics level, the constant acceleration model is the basic conceptual model used to understand the motion of objects. In the constant acceleration model, a set of tools or representations (such as graphs, motion maps, force diagrams, energy diagrams, equations, word descriptions, and pictures) help articulate and describe the defined set of relationships among concepts like acceleration, 
velocity, and position (disciplinary knowledge) and allows predictions to made about the motion of objects based on explanatory ideas like net force and energy transfer.

The idea that modeling is a central practice in science has been highlighted not only by science education researchers $([1,28,26])$, but has also been a central focus of science education policy in the United States and Europe. In the U.S., the Next Generation Science Standards (NGSS) list "Developing and Using Models" as one of the eight scientific and engineering practices that students should be learning across their K-12 education [30]. Similarly in the European Science Education: National Policies, Practices, and Research document, modeling is listed as being at "the core of doing science" and as a means to "give a deeper understanding of the nature of science and scientific inquiry processes" $[92,93]$. Therefore, modeling is recommended as a critical skill and competency for both students and science teachers.

In addition to forming the basic premise of the MI courses, MTS strongly influences how the content of the MI-Mech and MI-EM courses have been organized. Namely, that content is instead organized around 3-4 scientific, conceptual models per semester (i.e., the constant acceleration model) rather than the 10-12 chapters of an introductory textbook [26]. The following section on the Modeling Theory of Instruction then describes how this content is developed in the classroom through the Modeling Cycle.

\section{Modeling Theory of Instruction}

Modeling Theory of Instruction (MTI) applies MTS to the introductory physics classroom. If science is the process of developing, testing, applying and refining models, MTI then states that our students should then be learning physics by developing, 
testing, applying, and refining models because students learn best by constructing that knowledge and engaging in those practices for themselves [32, 26, 33].

According to MTI, students should be active participants in the work of constructing these models in class. As Brewe and Sawtelle describe, MTI guides how students participate through a variety of activities, including those that have students working with equipment and collecting data, problem-based activities, and small and large group discussions [27]. These participation cycles are integrated with the Modeling Cycle, which describes how the activities help students apply the modeling process $[26,27]$. The first step in the Modeling Cycle is to introduce the phenomenon being considered and figure out how to represent that phenomenon. Sometimes this requires students to use familiar or instinctual representations like drawing a picture or making a graph; however, sometimes this step requires students to construct a representation for a much more abstract idea. For example, when students are asked why a ball does not return to the initial height when bounced off the ground, students must build a representation that helps them reason about energy (such as the energy pie charts [13]) as the current tools they have in their model are not sufficient to describe why the ball does not bounce as high.

After introducing the phenomenon and representations, students are asked to intentionally coordinate between the new representations and those with which they are already familiar. Class time is then spent talking explicitly about the features of the various representations and how they relate to one another. These first two steps serve as the "development" of the model in the modeling cycle. Once students have a working model, they are asked to apply their model in a variety of different contexts. For example, they could be asked to apply the constant acceleration model when a 
ball is thrown upward, when the ball comes back down, when a train is speeding up on a track, etc. From the application of the model to many different situations, students are asked to abstract and generalize about the features of the models (e.g. What is similar about the models even though the context is different? What changes about the models when the context is changed?). This abstraction and generalization step is important because it creates a basic model that students can apply for any constant acceleration phenomenon.

The final step in the modeling cycle is refining the model, which is two-fold. First, this step requires students to recognize the assumptions that they have made in creating their model and the limitations that their model has in describing, predicting, and/or explaining a phenomenon. Second, students are often pushed in this step to consider what would happen if a certain assumption was relaxed or invalidated or what would happen if their model was applied in a new context that had previously not been considered. In the ball bouncing example, students have up until this point constructed a fairly thorough constant acceleration model that describes and predicts the motion of objects, which lets them describe the ball before and after the bounce but does not help them understand why the ball does not return to the same height. In trying to apply the constant acceleration model to this context, students recognize a limitation to their model (not being able to explain what is happening at the bounce), and it motivates refining the constant acceleration model to now include energy. Thus, starting the modeling cycle over with the question of how do we represent energy in the constant acceleration model. There can also be times where rather than refining the current model, starting over from scratch and building a new model is warranted, for instance, when transitioning from constant acceleration 
motion to oscillatory motion. Thus, students are guided through this modeling cycle multiple times in the semester - either as a means of adding a new aspect to the current model (i.e, adding energy to the constant acceleration model) or as a means of creating a new model for a different set of phenomena.

Through the modeling cycle students are building conceptual models and practicing the authentic skills that scientists use on an everyday basis.

\section{Modeling Discourse Management}

The final piece of the MI framework - Modeling Discourse Management (MDM) serves as a set of strategies that instructors use to promote students' participation in the construction, validation, deployment and revision of conceptual models [34, 35]. Practically, MDM describes the day-to-day activities in the Modeling Instruction classroom. On each activity, whether it be a experimental activity or an analytical activity, students are asked to work in small groups of three students. At the end of the activity, they are then prompted to answer three questions on a shared 2' $\mathrm{x}$ 3' whiteboard: 1) What did you learn? 2) What rules can you make? and 3) What is still unclear or what do you still have questions about? If the activity has asked students to create a model, they may also have a second whiteboard with their model of the phenomenon, including the various representations and assumptions that they have made.

After working on the activity or part of the activity in their small groups, students take their whiteboards to a larger group discussion, referred to as "Board Meeting", which is made up of approximately 8 of the small groups. In the Board Meeting, students present their ideas as well as what rules or patterns they are seeing from their 
work at that point. The Board Meeting also provides students with the opportunity to compare and contrast their findings with the other groups and to come to consensus on what they are seeing and how they can describe and explain the phenomenon. This discussion is meant to be lead by the students - with students doing the majority of the presentation, the consensus building, the question asking and the question answering.

The role of the instructor in the Board Meeting is then to guide the discussion, rather than lead it. Sometimes it may be necessary for an instructor to step in as a content expert to answer questions or prevent the discussion from going down an unproductive tangent; however, the goal of the board meeting is to have students discussing, critiquing, and exchanging their own findings. In the activity time prior to the Board Meetings, the instructors are walking around the classroom and interacting with groups. If there is a critical discussion point that students are missing in this work, the instructors can also use this time to discuss that point with a small group of students (referred to as "seeding" an idea) so that it comes up in the larger Board Meeting. Seeding is especially important when transitioning to a new idea or when introducing a new representation, as students often need to be pushed to think about these new areas.

While the goal of the activity and board meeting often changes depending on where in the Modeling Cycle the class is, the repeating pattern of an activity or part of an activity followed by a board meeting is consistent through the course.

\section{Role of Representations within the MI Framework}

Within the Modeling Instruction theoretical framework, representations play a critical role in how students build, apply and evaluate their models. As we outlined in the 
previous sections, the first two steps in the Modeling cycle are to: 1) figure out how to represent the phenomena, often coming up with new representations to describe the features needed, and 2) coordinate those representations with one another [26]. For example, this would mean that in a student's model, their word description of the motion should align with their force diagram, and both should also match their equations for net force and kinematics. Within the curriculum materials, the practice, coordination, and application of these representations is built into every activity. Furthermore, students are always asked in the activities and homework to "create a model" of the phenomenon, which means they are presenting and assessed on creating a coordinated set of representations as their work. This means that there is explicit class time and instruction in the MI courses devoted to helping students understand, use and apply the many representations used in physics.

\subsubsection{Use of Multiple Representations}

Beyond the Modeling Instruction framework, many researchers from physics education and STEM in general have recommended using multiple representations in the classroom [3, 50, 51, 52, 26, 53, 47, 54, 15]. Rosengrant, Etkina, and Van Heuvelen summarized work on multiple representations in Physics Education Research in their meta-analysis, which suggests that multiple representations improve students' conceptual understanding and ability to solve problems [7]. Specifically, De Leone and Gire found that students who use non-mathematical representations in addition to equations were more successful in solving problems [49]. Cox and Brna and Tabachneck, Koedinger, and Nathan further emphasize students' success when using multiple representations in other STEM disciplines [12, 48]. 
As Ainsworth summarized, multiple representations can serve a variety of purposes for students' learning, including: serving complementary roles, constraining interpretation, and helping construct deeper understanding [5]. Complementary representations allow students to switch between different visualizations (for example, a graph versus a table), which highlight different salient features that students may otherwise miss [5]. Having complementary representations can also allow students to take multiple pathways to a solution or to use the tools that make the most sense to them. Constraining representations, instead, serve a different purpose in that they help students build off of familiar representations to those that are more abstract $[5,9]$. For example, drawing a free body diagram can help one to reason about both the magnitude, direction, and number of forces acting on an object, which helps dictate and constrain what the net force on an object should look like when written out as an equation. In addition to helping students reason abstractly [5, 10], breaking this process into multiple representations helps to reduce student's cognitive load while working through a problem[11, 12]. These representations can also serve as an evaluative check of the answer by looking for consistency between representations. If, for example, your graph indicates that the object should be speeding up, but your equation has a decreasing speed, this could indicate to students that they have made a mistake in their solution (i.e., forgot a negative sign on their acceleration or are a missing a force in their diagram). Furthermore, many of the representations used in physics are designed to help students build a deeper understanding of the concepts behind the equations $[5,14,19,8]$. For example, energy pie charts are designed to help students reason about how energy is conserved, how it is transformed from one form to another, and how those quantities depend on the choice of system [13]. These 
kinds of representations ask students to reason conceptually about a situation before moving to the more quantitative representations.

However, many researchers have found that representation use (both in terms of individual representations and multiple representations) is a skill that students have to develop - just presenting representations without scaffolding or motivation has been shown to be problematic $[4,11,16,17,18,22,68]$. For example, Heckler showed that students actually did worse on some force problems when asked to make a force diagram first, which could indicate that students see the drawing of the force diagram as a completely unrelated task that does not relate to finding the net force on an object [21]. Further, Kohl and Finkelstein found that students used multiple representations differently than expert physicists; namely, that students tend to use more representations in their problem solving while experts were able to move between representations more quickly and were more flexible in how they began their problem solving [19]. In chemistry, Cooper et. al. found that students struggle to construct and interpret Lewis structures, but that with curricular reform and dedicated instruction students' ability to create and understand these diagrams was significantly improved $[4,15]$. These studies indicate that if we want students to be able to effectively use multiple representations in their problem solving, they need more than a basic presentation of the $\operatorname{tool}(\mathrm{s})$.

As is highlighted in the previous sections, the modeling cycle is designed is to address many of these concerns. Representations are only introduced when they are motivated by the phenomenon and by the need to represent an aspect of that phenomenon that the model does not currently address. When a new representation is introduced, there are multiple activities that allow students to practice with and 
see the variety of applications of that representation, which are continually revisited over the course of the semester. By the end of the Modeling Instruction courses, we expect students to be able to use and coordinate between the representations in their models. In light of the curriculum modifications, primarily to the MI-EM course, a significant amount of that work was done to align the activities with this modeling cycle and focus on how representations are introduced and incorporated in the model. The following section outlines the specific changes made to MI-EM course and the challenges faced in this development.

\subsection{Curriculum Changes}

As of the Fall semester of 2015, the Modeling Instruction - Mechanics course (MIMech) had been fully developed, iterated upon, and revised for many semesters, including a robust set of instructor's guides for teaching the course as a result of funding from the National Science Foundation. The course was developed using the Modeling Instruction Framework (described above) and had recently transitioned from a classroom with 30 students to one with 75 students. In contrast, the Modeling Instruction - Electricity and Magnetism course (MI-EM) as of 2015 used materials that had been pulled from multiple research-based sources, including the Matter and Interactions textbook [45], Washington Tutorials [94], and the Maryland Physics tutorials [95]; however, they were not designed with the modeling framework. In the 2015 courses, these materials were used with a similar MDM to that described above, primarily that students were asked to complete the activities, use whiteboards, and participate in board meetings as the central component of the course; however, these activities and the MI-EM curriculum as a whole did not follow the Modeling Theory 
of Instruction or the Modeling Theory of Science, meaning the activities were not centered around the modeling cycle and the curriculum was not centered around the basic models in EM.

These discrepancies (in addition to departmental requirements) motivated a significant re-design of the MI-EM curriculum with the primary goal of aligning the curricular materials with all three parts of the Modeling Instruction framework including designing the curriculum around four basic models (electric, circuits, magnetics, and optics models) and emphasizing the modeling cycle across the different activities. In addition to these large curricular goals, we also designed the activities to incorporate representations more consistently between the MI-Mech and MI-EM courses as well as to help transition students who did not take MI-Mech into the MI-EM course, based on our previous study of students' representation choices from the Spring 2015 [96]. The subsequent sections describe in detail the changes that were made to the activities and course for the Spring 2016 semester.

\subsubsection{Changes made to EM}

We created the MI-EM course with the primary focus being aligning the curriculum with the Modeling Instruction framework and creating a course where students are able to develop the general models in EM. In the development of the curriculum, we focused on four general models, which we will refer to as the electric, circuit, magnetic, and optics models. A summary of the content topics covered in each model is shown in Figure 11, where the left side of the figure shows the topics covered in each model during the Spring 2015 semester (prior to the curricular changes) and the right side shows those covered in the Spring 2016 semester after the curricular changes. Notes 
are included that highlight specific changes made, and the blending between models emphasizes the changes made to the model transitions.

Within the first two models (the electric and circuits models), we intentionally ordered the topics to follow the "energy first" thread established in the MI-Mech course [13]. For example, in the electric model, we introduced and discussed electric potential and electric potential energy before talking about the electric field and electric force. Emphasizing energy first was done for multiple purposes: 1) to establish energy as a valid approach to solving problems, which has been shown to be an underutilized method by students in introductory physics [97] and 2) to emphasize the commonalities between the mechanics and EM content (as well as the MI-Mech and MI-EM courses).

In addition, each classroom activity within the model followed the modeling cycle from the Modeling Theory of Instruction. The first activity (or activities) were designed to introduce a phenomena and build a conceptual understanding of what happens. For example, the introduction of the idea of charge is built around an activity using clear sticky tape. In this activity, students create "upper" and "lower" tapes and investigate how those pieces of tape interact with one another. From this investigation, students are able to determine that there are two types of charge (where opposite charges attract and similar charges repel) and that the strength of the interaction is both proportional to the amount of charge on each piece of tape and inversely proportional to the distance between them. The conceptual ideas listed above form the beginning of the electric model, based on the electric interaction between charges.

After introducing a phenomena, there are often new representations needed to be able to describe or visualize what is happening in a given situation. For example, 


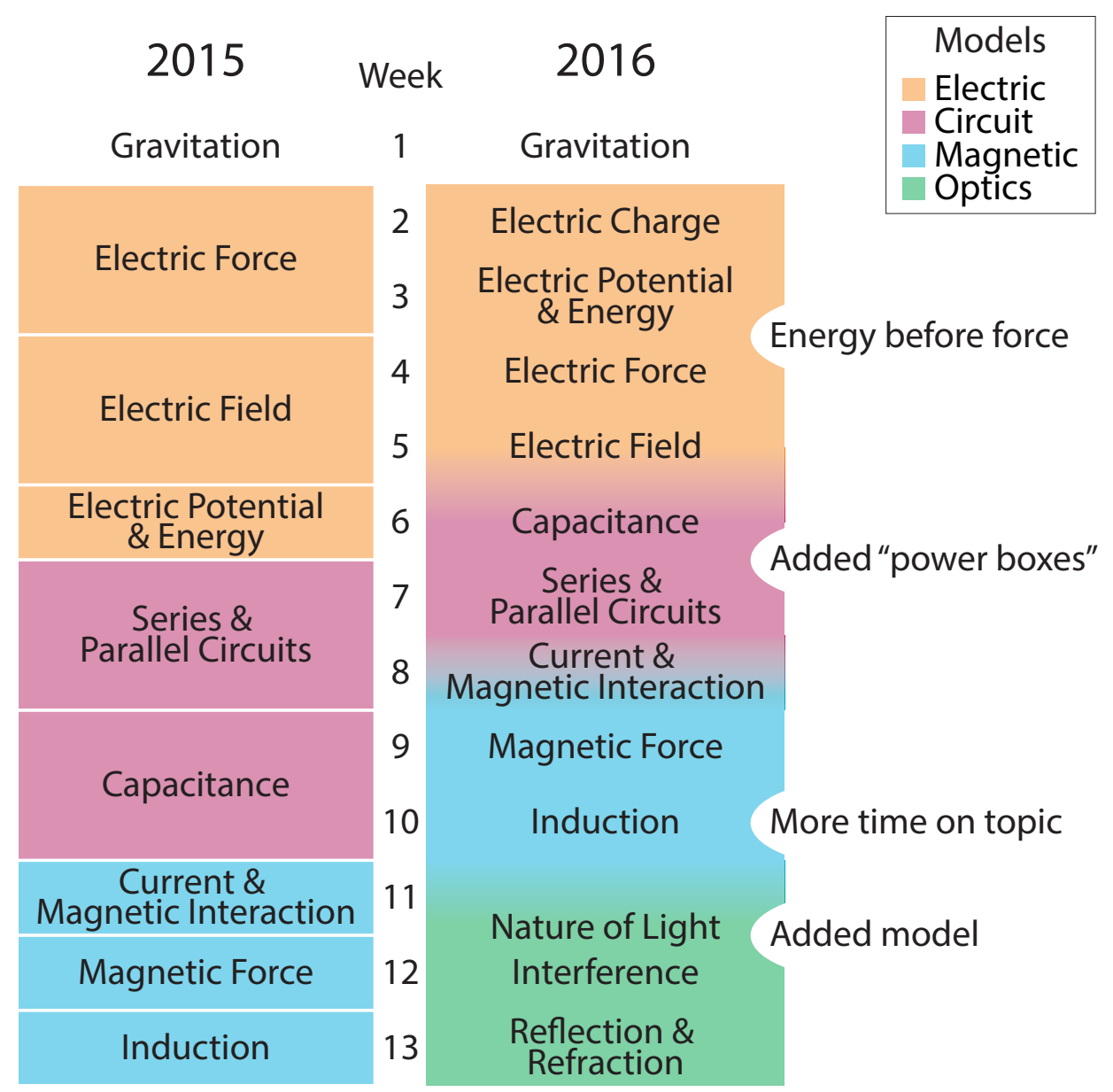

Figure 11: Content comparison of MI-EM between Spring 2015 and Spring 2016. This figure compares the physics topics covered in each model during the Spring 2015 semester (prior to the curricular changes) and the Spring 2016 semester (after the curricular changes). Notes are included on the right that highlight specific changes made to the Spring 2016 semester as well as blending between models to emphasize the changes made to the model transitions. 
after introducing electric potential energy, students are faced with a positive charge attracting a negative charge where it appears that the electric potential energy increases and that the kinetic energy of the system increases, which would appear to break energy conservation. Students must then reconcile the idea of what it means to have a negative potential energy and how best to represent this idea as the current tool that they have for energy - Energy Pie Charts - does not easily show negative energy. Having a negative electric potential energy motivates the use of Energy Bar Charts [53], which become a new representation for students to use in the course. The subsequent activities then focus on making sense of how Energy Bar Charts are similar and different from Energy Pie Charts, when would Bar Charts be a better choice to represent the energy and when would Pie Charts be better, and how do the Energy Bar Charts coordinate with the other representations that students have used at this point (e.g. system schema, pictures, words, etc.). At this point, we then ask students to apply their model (now including energy bar charts), to a variety of situations, which typically takes the form of asking each group of students to create a model for one or two different situations. In the following board meeting, students are then asked to present each of their models and, as a group, to compare and contrast the variety of situations. This discussion leads to the generalization of the model what are the common features, what features are context dependent, and what are the assumptions that are built into the model.

In the final step of the model building process, students are asked to refine their model based on what they found to be missing from their generalization. For example, after students have applied the Energy Bar Charts to the variety of situations, we ask them to then quantify the electric potential energy between two charges. At that point 
in the semester, their electric model does not have a mathematical representation of the electric potential energy or the electric interaction. Considering what is missing pushes students to consider quantifying energy and developing an equation for electric potential energy, which then begins the cycle over again. In this way, students are able to build upon and add to their model of what happens when charges interact, later adding the ideas of electric potential, electric force, and electric field in a similar process.

Another way that this refinement step can manifest in the curriculum is to push students to apply their model to a context that they have previously not considered. Typically, this alternate context breaks an assumption that students had previously been making (either explicitly or implicitly) and creates an opportunity to either try to add onto the previous model or to create a new model. In the course sequence, the refinement step in the modeling cycle provides the critical transitions between the general models. For instance, in moving from the electric model (which is primarily focused on electrostatics) to the circuits model, we ask students to consider what would happen if you had two parallel sheets of charge connected by a wire. From the electric model, students are familiar with and can describe what happens when there are two parallel sheets of charge; however, asking them to describe the process of what happens when they are connected by a wire pushes them to consider a situation now with moving charge. In this case, the new context then motivates the development of the circuits model (which granted, heavily relies on much of the electric model). In aligning these transitions with the assumptions and limitations of the model, we heavily emphasized the role of assumptions in evaluating and creating a model of the physical phenomena. 
To address the results suggested by the our previous work [96], we also added additional scaffolding to introduce students who did not take MI-Mech to the course structure, outline the representations that they may not have seen in a traditional mechanics physics course (like the system schema [8] and energy pie charts [13]) and what is meant by a model in this course.

In summary, the significant changes to the MI-EM curriculum were to: organize the content around the four general models in EM (adding the optics model), build the activities explicitly around the model building cycle (including explicit activities about introducing and coordinating representations, as well as a heavy emphasis on refining the models), follow a conceptual and energy-first focus from MI-Mech, and create an emphasis on model evaluation and limitations.

\subsubsection{Changes made to Mechanics}

In addition to these changes in the MI-EM course, we also made some adjustments to the MI-Mech curriculum to better lay the foundations for the material covered in MI-EM. One of which was to modify the section on work to better emphasize the relationship between potential energy, work, and forces. Having this relationship highlighted in the MI-Mech course allowed us to build off of these ideas to relate not only electric potential energy to electric force, but also electric potential to electric field, and justify why magnetic fields do not do work on charges. The other significant change was to add three explicit activities that relate oscillatory waves, simple harmonic motion, and circular motion. This was done to lay some of the foundations for the optics model in EM and to better relate the concepts between springs, 
oscillations, and circular motion. These areas were added to help promote coherence between the MI-Mech and MI-EM courses and content.

\subsection{Research Questions}

Since students' representation use and coordination is one of the main goals of the course, a central part of their model development and application, and a strong focus of the curriculum modifications, we aver that we should evaluate the curriculum on how it is impacting students' representation use. Thus, our primary research question is: can we see the effect of these curriculum changes in the number and variety of students' representation choices across the semester?

From our previous work [96], we found that there was a significant difference between students' representation choices depending on the context of the question (whether it was a mechanics context or an EM context) and on the type of mechanics course that students took (MI-Mech or a lecture-based mechanics course). Therefore, we also ask how are the number and variety of students' representation choices impacted by the type of mechanics course they took and the context (mechanics or EM) of the problem type?

\subsection{Methods}

In the following sections, we describe the survey that we developed to answer these research questions, the data we collected, and the statistical tests used in our analysis.

\subsubsection{PSRUS Survey}

To address these research questions, we designed a modified card-sort survey called the Problem Solving and Representation Use Survey (PSRUS) that asked what rep- 
resentations students would choose for a variety of mechanics and EM contexts. The survey was 25 questions long, with 12 questions based on mechanics contexts and 13 based on EM, and asked students to indicate what representations they would use to solve each problem (the full survey is provided in the appendix). Along with the survey, students were given a list of 15 representations used in MI courses (shown in Figure 12): graphs, equations, words, assumptions, motion map, picture, system schema, free body diagrams, momentum vectors, energy pie charts, energy bar charts, circuit diagrams, right hand rules, potential lines, and field lines. This set of representations also included an "other" option, so if there was a representation that students did not see listed, they could write in what they would use. For every question, students were not asked to solve the problem, but simply indicate what tools they would use if they were going to do so. On any given question, students were free to list anywhere from one to all sixteen representations. For example, one question from the PSRUS asks "A frisbee is thrown straight up in the air with an initial speed of $3 \mathrm{~m} / \mathrm{s}$. Find the maximum height the frisbee reaches." We would expect a student to respond to this question with the representations they would use to solve this problem. For instance, a student could say they would use equations, a system schema, a picture, and energy pie charts to find the height of the frisbee.

In developing the survey, we purposefully designed the problems on the PSRUS to be open to multiple representations and to cover a wide range of topics from both introductory mechanics and introductory EM. As shown in the example above, the questions on the PSRUS also asked for a specific quantity rather than the typical "make a model" prompts suggested by MDM so as to not artificially push students to list all of the given representations. Additionally, many of the mechanics problems 


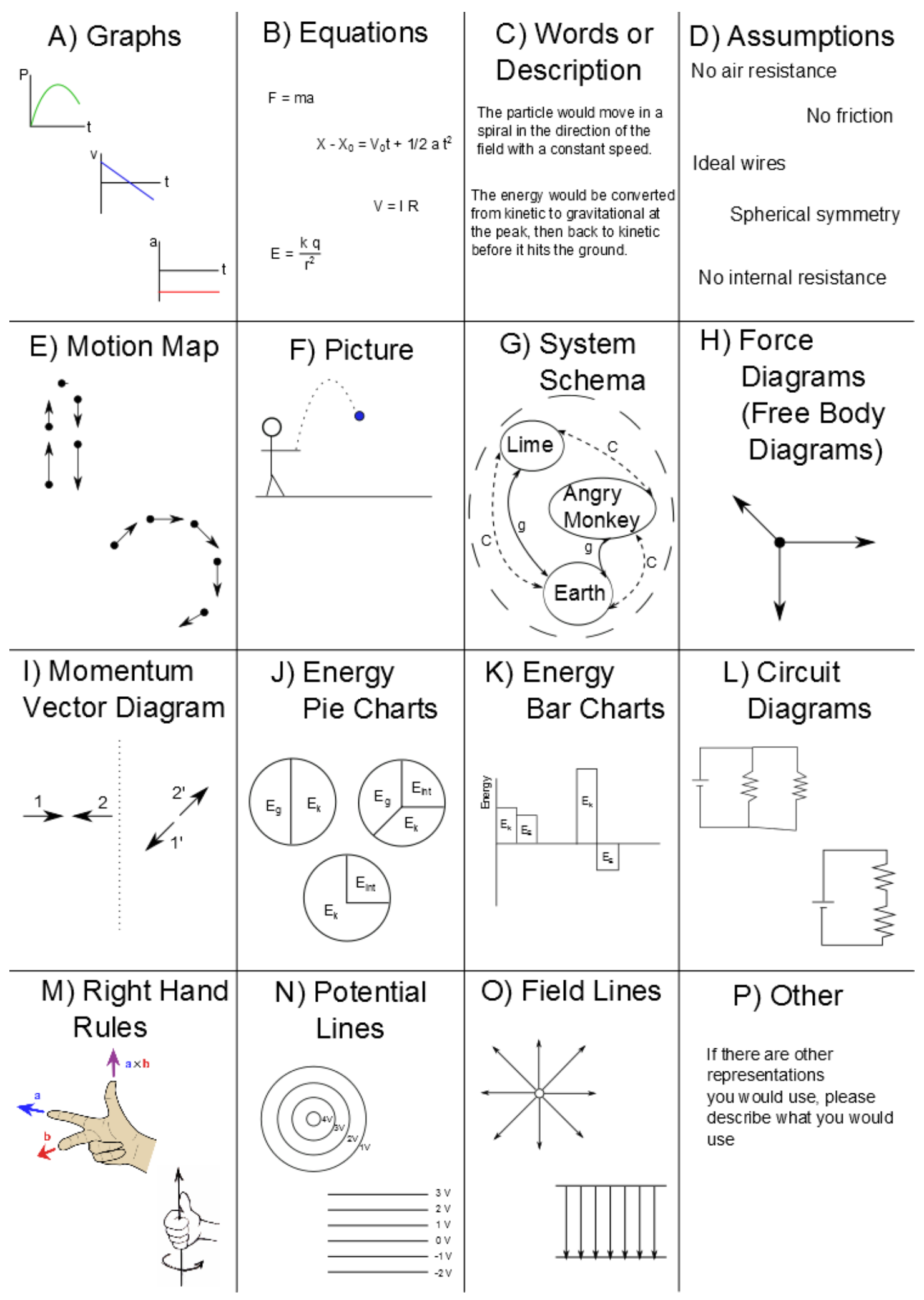

Figure 12: Representations given with the PSRUS.

This figure shows the list of representations that were given to students along with the Problem Solving and Representation Use Survey (PSRUS). 
were designed to have a parallel EM question. For example, there was force question about Newton's Third Law pairs in both mechanics and EM contexts (Questions 4 and 20 in the PSRUS Appendix). We compiled the list of 15 representations based on those explicitly covered in the MI-Mech and MI-EM curricular materials, in the instructor guides, and from conversations with MI instructors about what they expect in a student's solution.

It is important to note that the PSRUS was intentionally designed to examine what representations students are finding useful from the MI courses and in which contexts. Many studies have indicated that students' use representations in different ways than experts $[20,19]$; thus, we are intentionally not "grading" the survey or looking for the "correct" representations for each problem. Instead, we are looking at the number and variety of representations that students choose as an indication of the tools that they frequently rely on in a variety of physics contexts. That being said, there are some choices (like choosing circuit diagrams on a problem about a ball dropping) that would indicate that the students had not read the problems or were not taking the survey seriously. In such a case, the student's survey was removed from the data set. Since we were not assessing this survey based on correctness, we used a test panel of faculty, graduate, and undergraduate students at Florida International University to address the validity, reliability, and fairness of the survey during development [96]. Further discussion of the validity, reliability, and fairness of the PSRUS is given in the appendices. 


\subsubsection{Data Collection}

We administered the PSRUS as a paper survey in the MI-EM sections at Florida International University (FIU) the year before the curriculum was modified (Spring 2015) and the year after the curriculum changes (Spring 2016). FIU is a large, Hispanicserving Institution, with $61 \%$ of the student body identifying as Hispanic, $15 \%$ as White Non-Hispanic, $13 \%$ as Black, $4 \%$ Asian or Pacific Islander, and $7 \%$ as other minority groups [98]. In the MI-EM courses, most of the students who take the course are STEM majors, with the majority being engineering or biology/pre-medical majors.

In the Spring 2015 semester, the MI-EM course was taught in one section of 70 students by one physics faculty member, two graduate teaching assistants (TAs), and three undergraduate learning assistants (LAs). We gave the PSRUS at the beginning and end of the MI-EM course (along with other curriculum measures such as conceptual surveys $[99,100]$ and an affective survey [42]). Of the 70 person class (Spring 2015), we received valid pre-surveys from 69 students (24 Female/45 Male) and valid post-surveys from 60 students (20 Female/40 Male), with 58 of those students (19 Female/39 Male) submitting both pre- and post-surveys. The data reported below are from the set of 58 students who had matched pre- and post-surveys. Of the 58 students, 30 had taken MI-Mech as the prerequisite mechanics course for MI-EM, whom we will refer to as returning students (returning to the MI format); whereas, 28 had taken a traditional lecture/laboratory section of introductory mechanics for that requirement, whom we will refer to as new students (new to the MI format).

In the Spring 2016 semester, MI-EM was taught in two sections of 70 students. One section was taught by the same physics faculty member, one graduate TA (return- 
ing from the Spring 2015 semester), and two LAs (new LAs, previous MI students). The other section of the Spring 2016 semester was taught by a physics post-doctoral researcher (new to MI), two graduate TAs (one returning from the Spring 2015 and one new to MI), and three undergraduate LAs (two new LAs, one returning from the Spring 2015 course). Both sections of MI-EM used the same curricular materials, had combined prep meetings, developed common exams, and shared grading rubrics for all assignments. We again gave the PSRUS at the beginning and end of the MI-EM course (along with the same conceptual surveys). When we compared the results of the PSRUS, we found no significant difference between the number and variety of representations that students chose in the two sections for either pre- or post-PSRUS, when accounting for mechanics background (new or returning to MI) and mechanics/EM contexts. (For the full section comparison analysis, see the Tables 4-7 in the Appendix.) Therefore, we combined the two sections of MI-EM for Spring 2016 into a single data set. In the combined 2016 data set, we received valid pre-surveys from a total of 129 students (52 Female/77 Male), valid post-surveys from 121 students (51 Female/70 Male), with 113 of those students (48 Female/65 Male) matched across both pre- and post-surveys, where the following analyses are based off of the matched data. Of the 113 students, 91 were returning students (coming from the MI-Mech course) and 22 were new students (coming from the lecture-based mechanics course).

\subsubsection{Analysis}

From the PSRUS data, we first counted the number of representations that each student chose in total and divided this by the number of questions to get the average number of representations that each student chose per question. Since each student 


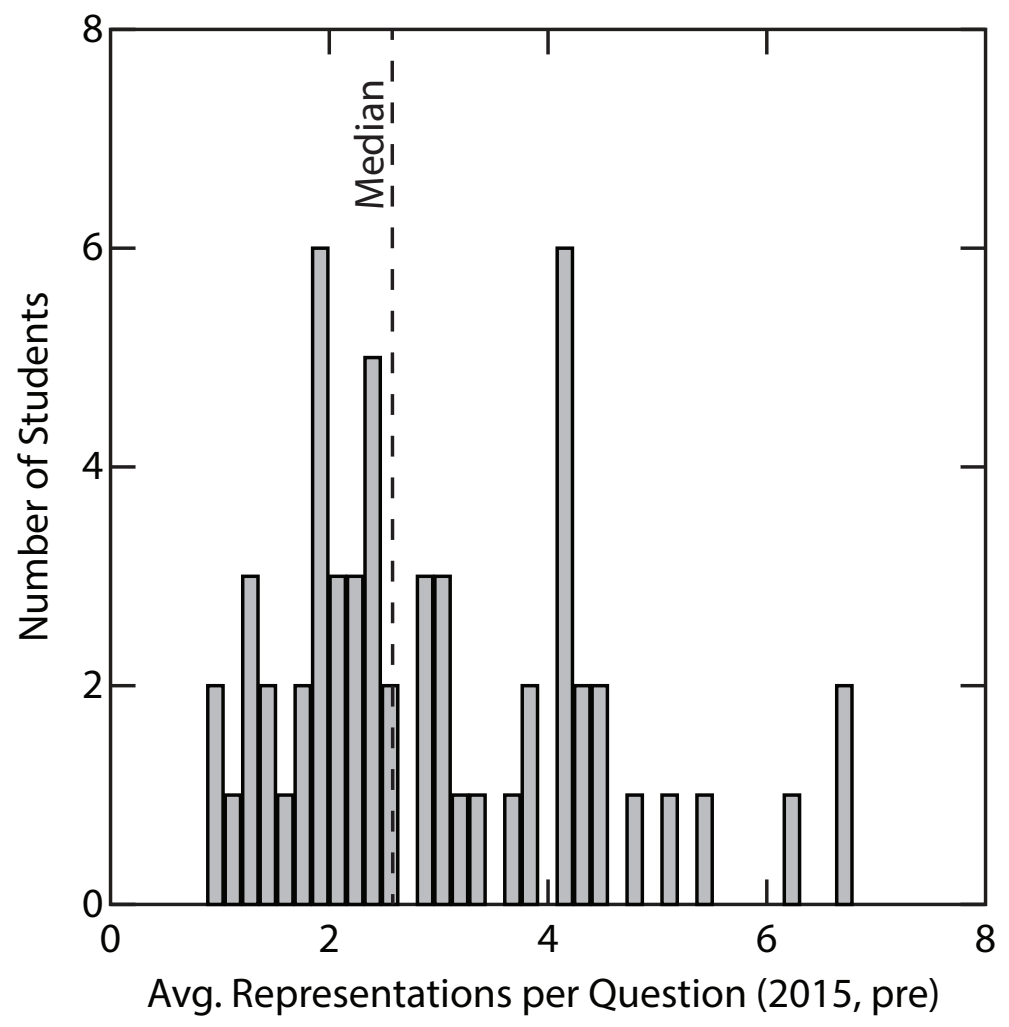

Figure 13: Sample distribution from PSRUS responses.

This distribution shows the average number of representations students choose per question on the pre-PSRUS in Spring 2015. The median of the distribution is marked at 2.56 representations per questions.

then has an average number of representations that they chose, we built a distribution of the average number of representations based on the entire class. For example, Figure 13 shows the histogram of the average number of representations chosen by students in Spring 2015 on the pre-survey.

From the histogram it is clear that the distribution of representations is not normally distributed. Instead, there is a floor at one representation and a right-skewed tail to the distribution. However, given the design of the survey, this is to be expected. 
Students are expected to list at least one representation for every question (whether it be words or an equation), which accounts for the lower bound. When looking at the higher end of the distribution, students could list any number of representations per problem, up to the 16 listed options that they were given. However, every representation is not applicable to every problem (i.e. circuit diagrams), so it is expected to have a wider spread in the distribution, based on what representations students see as applicable.

When we separate the data by question type (either all questions combined, only mechanics questions, or only EM questions) or when we separate by students (either all students combined, only returning students, or only new students), we see a similar distribution shape - right-skewed tail with a floor at one representation per question. Because these distributions are not normal, we choose to use median as the measure of center rather than the mean, as the mean is pulled more heavily off the center of the distribution when there is a skewed tail to the distribution. We also use non-parametric statistical tests when making comparisons between groups, namely, Wilcoxon-Signed-Rank (WSR) tests and Wilcoxon-Mann-Whitney (WMW) tests [73, 74].

We also looked at the variety of representation choices that student make by counting the number of times that a student would choose a given representation. Similar to the process above, we then created a distribution over all the students in the course for each of the listed representations. In contrast to distributions described above, the distributions for each representation varied widely in shape and in frequency. For this reason, we use Cliff's Delta Effect Size to distinguish different groups with regards to how often a representation is chosen [78]. 
In the subsequent sections, we outline the WSR and WMW tests, which we used to compare the number of representations that students chose, and the Cliff's Delta effect size, which we use to compare the variety of representations.

\section{WSR and WMW Tests - Number comparison}

The WSR and WMW tests are the equivalent non-parametric tests to the paired and unpaired t-test, respectively. Both the WSR and WMW tests are based on a rank-sum procedure to compare two distributions, which means that test is capable of distinguishing differences in the shape of the distributions and differences in the placement of those distributions $[73,74]$. In the cases where the distributions have the same shape, a significant result can be interpreted as a difference in the medians of the two distributions in the comparison [75]. As discussed above, the distributions that compare the number of representations that students choose all have the same distribution shape; thus, we will use WSR and WMW tests to compare the medians of these distributions. The WMW test is built on the assumption that the two populations are independent from one another, which we therefore use to compare the new and returning student responses and the 2015 to 2016 responses. The WSR test on the other hand allows for paired comparisons, which we use to compare pre to post survey responses and the mechanics to EM survey responses.

As we are making multiple comparisons (twelve 2015 vs 2016 tests, eight mechanics vs EM tests, twelve new vs returning tests, and twelve pre vs post tests), we used an alpha level of 0.00114 based on the Bonferroni correction of

$$
\alpha_{B 1}=\frac{0.05}{44}=0.00114
$$


Thus, we are testing each comparison with a $99.89 \%$ confidence interval, which gives a $95 \%$ confidence of the overall study of 44 individual tests. This alpha level, while certainly accounting for multiple tests, is the most conservative threshold for significance. Despite the conservative nature of the Bonferroni correction [76], we choose this as it accounts for the family-wise and per-family error rates on Type 1 Error[77].

\section{Cliff's Delta - Variety comparison}

To compare the difference in the types of representations that students choose between the 2015 and 2016 semester, we use Cliff's Delta effect sizes, which is again a rankbased non-parametric effect size that makes no assumptions about the shapes of the distributions [78]. Essentially, Cliff's Delta compares the number of times that a data point from Distribution $\mathrm{X}$ is larger than the data points from Distribution $\mathrm{Y}$ to the number of times that a data point from Distribution $\mathrm{Y}$ is larger than that from Distribution $\mathrm{X}$. This value is then divided by the total number of data points in both Distribution $\mathrm{X}$ and Distribution $\mathrm{Y}$ as shown in the equation below.

$$
\delta=\frac{\#\left(X_{i}>Y_{j}\right)-\#\left(X_{i}<Y_{j}\right)}{N_{X} * N_{Y}}
$$

In this notation, a positive effect would mean that Distribution $\mathrm{X}$ has a larger average value; whereas a negative effect would mean that Distribution Y has a larger value. If the Cliff's delta is zero, this means that the two distributions are overlapping or indistinguishable. (Cliff derives the error calculations in his paper for both the paired and unpaired tests [78].) In contrast to Cohen's D, Cliff's Delta has an upperbound of 1.0 and lower bound of -1.0 , which means that the interpretation of small, 
medium and large effects (corresponding to $\mathrm{d}=0.2, \mathrm{~d}=0.5$, and $\mathrm{d}=0.8$ by Cohen [79]) are shifted to $\delta=0.147, \delta=0.33$, and $\delta=0.474$ respectively [80].

Since we are interested in comparing the 16 representations, we use an alpha level of 0.003125 again using a Bonferroni correction.

$$
\alpha_{B 2}=\frac{0.05}{16}=0.003125
$$

Thus we are testing each representation at a $99.7 \%$ confidence interval so that the results of the overall comparison are at the rate of $5 \%$ error. Again, this is a conservative estimate; however, it allows us to be confident in the results we do see.

\subsection{Results and Discussion}

The graph shown in Figure 14 summarizes the results from the PSRUS over the Spring 2015 and Spring 2016 semesters. The leftmost region shows the results for the preand post-survey for all the questions combined; the center and rightmost regions show the results for the PSRUS questions separated by the EM and mechanics context. The red lines show the data from the Spring 2015 semester, and the blue lines show the data from the Spring 2016 semester (again with both sections of the MI-EM course combined). The dashed lines represent the responses from returning students and the solid lines show the responses from new students. Across all three columns, we see no significant difference between the new students responses from 2015 to 2016 on the

pre-PSRUS, which tells us that these students are entering the MI-EM with roughly the same average number of representations (about 2) chosen per problem. We do see a difference in returning students' choices on the pre-PSRUS; however, this is not 
entirely unexpected as we did make curricular changes to the MI-Mech curriculum in addition to those in MI-EM.

From Figure 14, we see two important results: 1) students use significantly more representations by the end of MI-EM, regardless of the question context or previous exposure to MI and 2) by the end of MI-EM in 2016, there is no difference in the number of representations that students are choosing based on whether they took MI-Mech (whereas in the Spring 2015 data there is).

Starting with the first result, we see that there is a significant increase in the number of representations from pre-post for all questions combined, for mechanics questions only, and EM questions only for both new and returning students in the Spring 2016 data by WMW tests (see supplemental materials for table of exact pvalues for each test). We would expect an increase in the number of representations for EM questions given that students are in the MI-EM course and are subsequently learning several new representations as introduced in the Modeling Cycle (e.g. potential lines, field lines, right hand rules, circuit diagrams, and energy bar charts). However, the fact that we see a similar increase in the number of representations for mechanics questions is surprising. The increase of representations on mechanics questions indicates that students are seeing these representations as useful to contexts that are outside of the content of the course and are recognizing these tools as applicable to ideas they have already learned. This result raises questions about how this work is related to the large of body of research on transfer, which indicates that transfer largely does not occur $[101,102,103,104,105,106]$. The fact that we see an increase in representations on mechanics questions further justifies the claim that MI-EM is impacting more than just content knowledge, but is also impacting how 


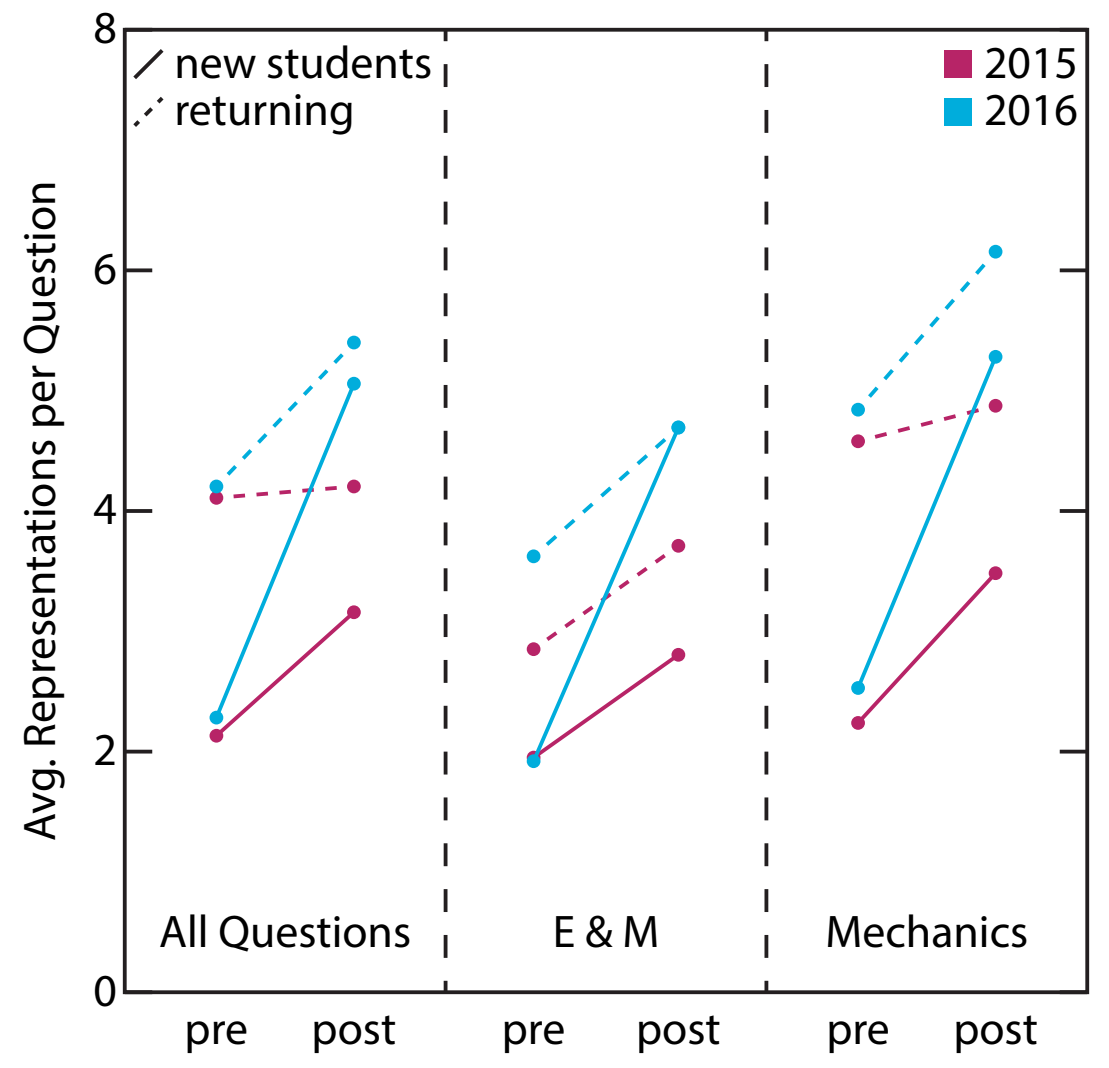

Figure 14: Summary of PSRUS results across MI-EM development.

This graph summarizes the results from the PSRUS over the Spring 2015 and Spring 2016 semester, which is split into 3 sections: all questions combined, only EM questions, and only Mech questions. The red lines show the 2015 data, whereas the blue line show the 2016 data. The dashed lines represent the responses from returning students and the solid lines show the responses from new students. 
students approach their problem-solving. Additionally, this increase in the number of representations chosen from pre to post is especially meaningful when compared to that of the Spring 2015 data. In every category across the board on the post-survey, we see that students from the Spring 2016 semester (after the curriculum changes) are choosing more representations per problem than their Spring 2015 counterparts. As one of the primary goals for the course is to teach students how to use multiple representations and to rely on these tools when creating a model or finding a solution to a problem, we interpret the increase in the overall number of representations as a positive outcome of the curricular changes.

Furthermore, we see no significant differences in the post survey between the number of representations that new and returning students choose, which is in contrast to the Spring 2015 semester where returning students tend to choose more representations than students who were new to modeling.

The fact that we no longer see this gap indicates that the new MI-EM curriculum is doing a better job of introducing new students to the variety of representations used in the course and helping them better see the applicability of those representations to not only the course context (as indicated on the EM questions) but also in other areas of problem solving (based on the increase in mechanics questions). This result is important as it says that all of the students coming out of the MI-EM course have access to and see the applicability of many representations in their problems, regardless of their mechanics course. In light of the literature that suggest using multiple representations helps students be more successful in their problem-solving $[3,50,51,52,26,53,47,54,5,48,12,49]$, this result would indicate that both the new and returning students have access to the same toolbox of representations and 
would have a more equitable chance of success, rather than favoring the returning students or putting the new students at a disadvantage because of their mechanics course.

Figure 14 shows that there is an overall increase in the number of representations chosen by students between the Spring 2015 and Spring 2016 semesters; however, it does tells us which representations students are actually choosing more frequently. To answer this question, we instead look at the number of times that students choose each representation in Spring 2016 compared to Spring 2015 using the Cliff's Delta effect size. A summary of these results is provided in Figure 15. In this figure, we compare the number of times a representation was chosen in the Spring 2016 post-PSRUS to that of the Spring 2015 post-PSRUS. In this case, a significant positive effect indicates that the representation was used more often after the curricular changes (in the Spring 2016 semester) and a significant negative effect would indicate that the representation was used more often prior to the curriculum changes. The large, medium, and small effects are indicated in green, orange, and red, respectively. The gray effects are those with confidence intervals that cross the zero line, indicating that the representation was not chosen more often in one semester over the other.

From Figure 15, the representations that have the largest increase in use from Spring 2015 to Spring 2016 are the words, pictures, assumptions, potential lines, and right hand rules. We hypothesize that these results correspond directly to the changes made to the MI-EM curriculum. As described in Section II, the Spring 2016 curriculum included a larger focus on limitations of models and how/when models are applicable. This means that there were more conversations in class and a heavier emphasis in the problems (both in class and on homework) on explicitly writing out 
the assumptions made for that model. In addition, with the increased level of math required in EM, there was also an increase in the curricular materials on being able to explain what those mathematical features were, which could possibly explain why students would choose words more frequently. In the curricular materials, one of the largest changes in the electric model was to continue the "energy first" thread that was established in the MI-Mech course. This meant that students saw electric potential energy and electric potential before they saw electric field and electric force, with the first three weeks of the course devoted to discussing gravitational and electric potential and potential energy. This focus on potential early in the course could account for the increase seen in the potential lines representation. Additionally, there was significantly more class time spent on working with sources of magnetic fields and an additional week on induction, including a formal lab report, which may account for the increased use of right hand rules in Spring 2016 compared to Spring 2015. In contrast, the increase in pictures was unexpected as there were not explicit curricular moves designed to focus on this representation; however, this could be a result of the fact that EM is much harder to visualize (when compared to mechanics).

That being said, there were instructional moves made to increase the role of system schema in the MI-EM course and to tie that representation back to what students learned in their mechanics course, but we do not see a significant increase in the number of times that this representation was listed. (This may be a result of the conservative $\alpha$ threshold used from the Bonferroni correction.) There were also many representations introduced in the MI-EM course that were more heavily emphasized and incorporated into the Modeling cycle that we do not see more frequently chosen, namely: field lines, energy bar charts, and circuit diagrams. The lack of change in 


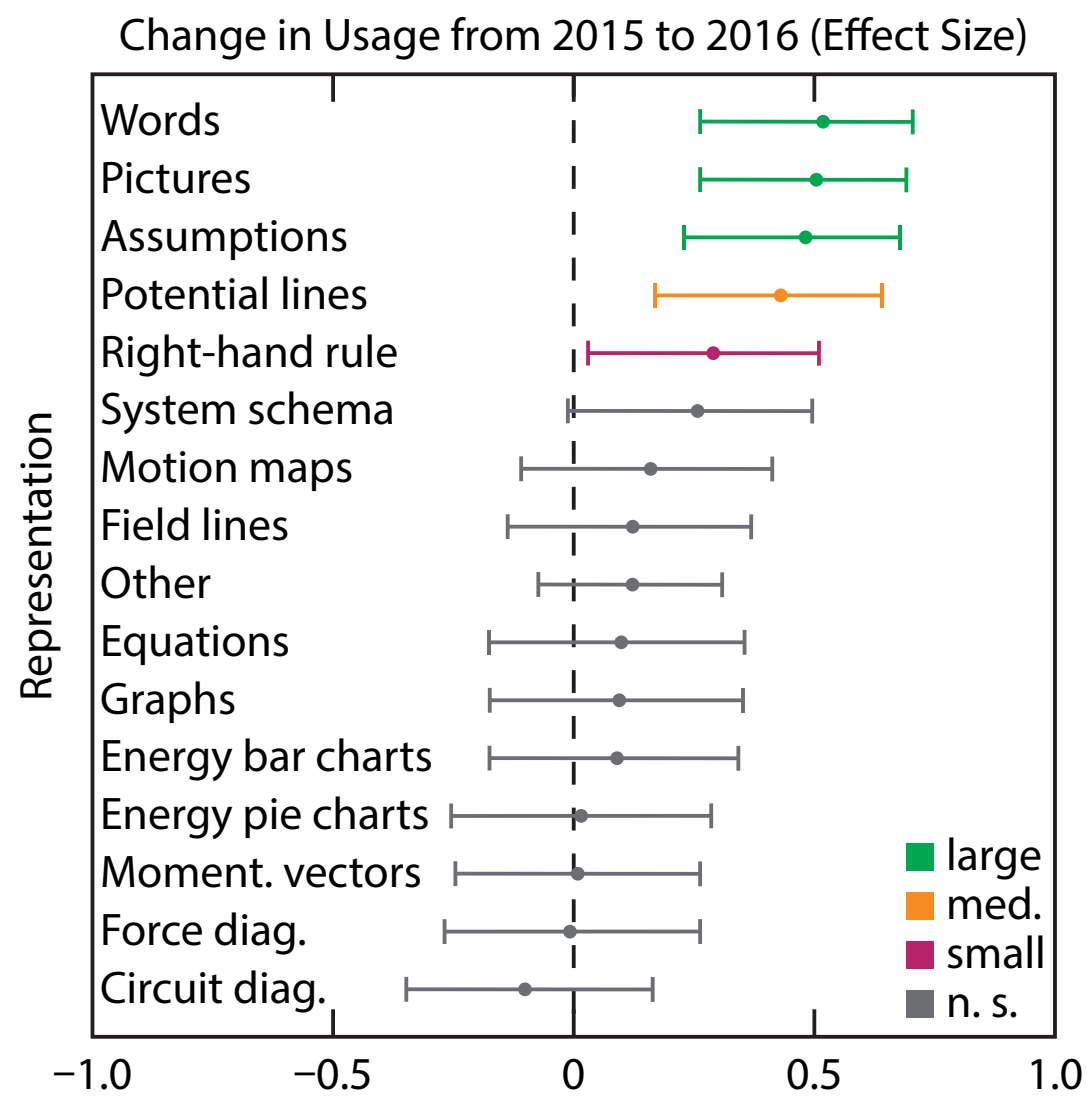

Figure 15: Cliff's Delta effect sizes across MI-EM curriculum development. This figure summarizes the change in usage from 2015 to 2016 of each representation listed on the PSRUS, using Cliff's Delta Effect Size. A positive effect here indicates that the representation was chosen more frequently in 2016 than in 2015; whereas a negative effect indicates the reverse. The effects shown in green indicate a large effect, those in orange are a medium effect, those in red are a small effect, and those in grey are non-significant. 
these representations could mean that the changes made to the curriculum did not help students understand those particular representations or the changes made in the curriculum were not large enough to impact when students viewed those representations as applicable.

Finally, we also created a new representation for the Spring 2016 MI-EM curriculum, called "Power Boxes," to highlight how power in a circuit is related to the current and voltage of each element (shown in Figure 11) [14]. However, the introduction of Power Boxes does not account for the increase in chosen representations seen in Figure 14. Power Boxes were not explicitly added to the PSRUS in the Spring 2016 to preserve the comparison across the Spring 2015 and Spring 2016 semesters, but students in the Spring 2016 semester could write the representation in as the "other" option. As shown in Figure 15, the "other" option does have a small positive effect as a result, but its confidence interval crosses zero and is not significant. Thus, the increase in the total number of representations chosen in the Spring 2016 semester is not due to the additional Power Boxes representation, but due to an increase in the students' choice of the representations that were already a part of the PSRUS and MI curricula.

\subsection{Conclusions}

To address our research questions about the effect of the curriculum changes on the number and variety of representations that student choose, we created the PSRUS which was given out in both the semester prior to the curriculum changes (Spring 2015) and the semester following the changes in the curriculum (Spring 2016). We found that in the semester following the changes in the curriculum, students used 
more representations across the board - independent of content area (mechanics or EM questions) and type of mechanics course (returning or new students to EM), as shown in Figure 14. Additionally, we found that the difference between the number of representations chosen by the returning students and the new students no longer detected by the post-2016 survey, which indicates that the new curriculum is introducing new students to MI structure and representations better.

Being able to use, coordinate, and switch between multiple representations is often seen as an expert skill in science and is typically an implicit goal of many physics courses. Furthermore, studies have shown that students who use multiple representations are more likely to be successful in their problem solving [48, 12, 49]. The MI framework and course design make this an explicit goal of the course and have integrated this through the activities, homework, exams, and instructor guides of the course. Thus, we interpret the increase in students' representation choices as a positive outcome of the curriculum modifications. This would indicate that students see these representations as applicable in more contexts and to more problems than had previously been the case.

Furthermore, this result suggests that as developers of second-semester or secondsequence curricula, we have to consider the effects on students who did not take the first semester course. In reformed curricula, like MI, the format of the course often looks completely foreign to students (whiteboarding, board meetings, making models, etc) and the ways in which we ask students to engage are different. In the second semester (MI-EM), students who are already used to the reformed course (like MI-Mech) have an advantage over the students who did not take the reformed prerequisite courses in that they understand more about what "the point" of the 
course is, how they are being assessed, and how to be successful in such a course. This work has shown that explicit design in the materials to help students enter new classroom formats (like MI) can help catch them up to the same level (in terms of representation choices) as returning students.

When we examined what representations students were choosing more often in the Spring 2016 semester (in Figure 15), we found that students were choosing words, pictures, assumptions, potential lines, and right hand rules more frequently after the curriculum design as compared to before. These representations tie directly to changes made in the MI-EM curriculum, such as the push to keep the energy-first thread from MI-Mech and increasing the amount of time spent on magnetics. Of the representations that we see increases in, words and assumptions tie into the largest changes made in the curriculum. First of which was to articulate and discuss the limitations of the models being developed, which placed a large emphasis on the assumptions that were built into the model. Second was to highlight that students should be able to explain in words the parts of an equation and how the variables not only relate to each other but how they relate to the real world. The fact that we see such large increases in these representations shows that students are valuing these pieces and listing them as part of their representation process.

While we do see significant increases in these representations listed (words, pictures, assumptions, potential lines, and right hand rules) as well as an increase in the total number of representations overall, these results also indicate areas that could still improvement. For example, we still see students choosing more representations in mechanics contexts over EM contexts. Since EM is generally "invisible," we would not expect students to choose fewer representations in EM. This could indicate an 
interesting area of future study as to why students choose fewer representations in EM. There were also several representations that were integrated more thoroughly through the curriculum (like energy bar charts and system schema) that we do not see significant changes for when compared to the semester before the curriculum changes. These could indicate areas of the curriculum that would need further work or consideration with regards to introducing and coordinating representations, or perhaps students' usage of these representations is already saturated.

Finally, this work has important implications for curriculum developers, instructors and researchers. We argue that as a community we should be evaluating curricula based off the goals of that course, which can certainly include conceptual measures like the CSEM [99] or FCI [39]; however, these surveys are not capable of assessing all course goals. For example, a multiple choice conceptual survey is not designed to assess the kinds of representations that students find useful, just as the PSRUS cannot measure how students' attitudes toward science change over the semester. In this work, we present a means of evaluating the MI-EM curriculum based on a goal of the course (fluent representation use) that was integrated into the curricular materials from the Modeling framework. We recognize that this is not the only means of evaluating a curriculum, but that the PSRUS has provided insight into the curriculum development that we would not have seen by other means. We would encourage others to think about the goals for their courses beyond increasing students' content knowledge, how they would be able to assess those goals, and to consider multiple means of curriculum evaluation for a course. 


\section{EXAMINING CONCURRENT REPRSENTATION CHOICES IN UNIVERSITY MODELING INSTRUCTION}

\subsection{Abstract}

As representation use is a critical skill in physics to communicate, visualize, and construct knowledge in physics, this paper focuses on which representations students use concurrently during problem solving. We situate this study in the University Modeling Instruction (MI) classroom context because the MI curricula spends explicit class time devoted to generating, understanding, interpreting, and using multiple representations as part of the model building process. MI is a two-semester, introductory physics course sequence, with the first covering introductory mechanics (MI-Mech) and the second covering introductory electricity and magnetism (MI-EM). In this paper, we address the question of how students' concurrent representation choices are different between the mechanics and EM contexts. To answer this question, 121 students from two sections of the electricity and magnetism Modeling Instruction course (MI-EM) in Spring 2016 were given a survey of 25 physics problem statements at the end of the semester, covering both Mechanics and EM content. Rather than asking students to solve every problem, they were asked to simply list which representations they would use. This list of representations was treated as a bipartite network of problems $\mathrm{x}$ representations. We then constructed two networks for the mechanics and EM questions from students' responses on the survey. We filtered these networks using a disparity filter to determine the "backbone" or most important connections in these networks and compared the mechanics and EM filtered networks to determine what representations students frequently rely on together in each context, what representations feed into others, and what representations serve as "connectors" between the 
various representations. We found that that on mechanics questions, MI-EM students repeatedly use a common set of representations, whereas in EM they have a wider array in their concurrent representations across the problems. We also find that in both mechanics and EM contexts pictures play a critical role in connecting different representations. Ultimately, these results have implications for further curriculum development and open up many future directions for study.

\subsection{Introduction}

In physics and in science more generally, representations are fundamental tools that we use when problem solving, sharing or presenting ideas, or constructing knowledge about a phenomenon $[6,5]$. These representations (such as equations, graphs, pictures, word descriptions, force diagrams, etc.) often highlight salient information, summarize large amounts of data, and/or reflect otherwise invisible characteristics of objects at all levels from introductory textbooks to peer-reviewed, scientific journals. Being able to create, interpret, and reason with many representations is a critical part of what it means to do science.

In the classroom, representations form the basis of what students are primarily evaluated on and serve as tools for their problem solving, and thus, has been a prominent research area in Physics Education and STEM Education more generally. For example, there has been extensive research in students' understanding of graphs in a wide variety contexts, from introductory mechanics to thermodynamics to upper-division electricity and magnetism $[55,56,57,58,107,20,59]$. In addition to graphs, research into how students understand equations [60, 61, 62, 47], force

diagrams $[21,51]$, pictures [63], and energy diagrams $[53,64,65,13]$ has been used to 
develop activities, curricular materials, and professional development workshops for instructors to incorporate these representational tools into the teaching and learning of physics.

However in physics, students are frequently encouraged to use more than one representation to solve or understand a problem. For example, on a force problem, instructors (and curricular materials) will recommend that students draw a picture and create a force diagram before writing out an equation and solving for the acceleration. Thus, how students work with and coordinate between multiple representations like these has also become a research focus [7]. Ainsworth argues that multiple representations serve multiple purposes and ultimately benefit students in problem solving by either serving complementary roles, by constraining interpretation, or by helping students construct deeper understanding [5]. For example, if students don't understand an equation they could construct a complementary graph to help them understand the phenomenon. Complementary representations (such as graphs and equations) can aid students by providing multiple pathways to a solution, often highlighting different salient information or allowing the student to work with tools they are more familiar with or have a preference for. Similarly, multiple representations have been shown to reduce the cognitive load of students by dividing the given information and serving as intermediate steps in problem solving $[11,12]$.

Alternatively, constraining representations serve to help students build from familiar representations to those that are more abstract through an inherently shared feature of the representations $[5,9,10]$. For instance, a system schema in Modeling Instruction (MI) is used to articulate the objects, interactions, and system boundary for a given problem [8]. In this representation, the interactions can be directly related 
to a force - if there is an gravitational interaction between the Earth and a ball, then there is a gravitational force from the Earth on the ball and also a gravitational force from the ball on the Earth. Thus, the system schema as a representation can be used to constrain the force diagram (as a representation) for a given object. Namely, if an object has three interactions in the system schema, then the force diagram of that object should also have three forces. If a student ends up with 4 forces in their force diagram, this indicates that they either have missed an interaction in their system schema or have included a force that does not belong for that object. In this way, constraining representations explicitly ask students to coordinate their representations and can serve as an evaluative check for students of their work (without the aid of an instructor).

Finally, many representations are designed to help students construct a deeper understanding of the problem or phenomenon at hand, often by providing intermediate steps or focusing on different conceptual pieces [5, 7]. For example, energy pie charts were designed to help students keep track of energy storage, energy conservation and energy transfers within a system [13]. In creating the energy pie charts, students are asked to qualitatively think through how energy is stored at the beginning of the problem, how that energy is transferred, and whether energy is added or removed (or neither) from the system. The intention of this representation is to ask students to reason conceptually about energy before calculating any energy quantities. Energy pie charts can then be used to build energy conservation equations and are constrained by the system schema (especially the system boundary) that students choose. Beyond energy pie charts, there are many representations in physics that are 
specifically designed to encourage a deeper understanding of a concept (i.e. force diagrams, momentum vectors, field lines, etc.).

As alluded to in the previous example, many representations do not serve a single purpose and can be used in conjunction to aid students in their problem solving. Thus, many researchers and instructors have recommended using multiple representations in the teaching and learning of physics $[3,50,51,52,26,53,47,54]$ and have shown that multiple representations increase students ability to solve problems $[48,12,49]$. However, many studies have indicated that multiple representation use is a skill that students develop and is not something that they inherently know how to use $[4,11,16,17,18]$. For example, Heckler and Kuo, Hallinen, and Conlin found that prompting students to draw a force diagram actually corresponded to students performing worse on a problem rather than improving their solutions, which indicates that these students were not relating the force representation to the net force equation as intended by the instruction $[21,22]$. This result is further supported by work from Kohl and Finklestein and Maries, which indicate that students use representations differently than experts in physics $[19,20]$. To address these concerns, there has been a recent focus in physics and STEM curricula on helping students use and coordinate representations $[26,94,86,12,15]$.

In physics education, research on students' representation use has primarily focused on either students' ability to use a single representation or how students coordinate between two specific representations. In the work done on multiple representations, the majority of studies focus on the number of representations that students use in solving a single problem (particularly in reformed physics classrooms) and how that correlates to their success on that problem $[7,49,67,19]$. While this work has led 
to the important results previously discussed, these studies are not able to show what representations students broadly see as connected and useful together in their problem solving. Based on the benefits outlined by Ainsworth of using multiple representations to coordinate, constrain, and deepen understanding [5], we designed this study to examine the representation choices that students make in a wide range of problems across the introductory physics content. This paper outlines how we applied network analysis to examine what concurrent representation choices that students make and resultant patterns we see in students' problem solving.

\subsection{Study Context}

We chose to conduct this study in the Modeling Instruction - Electricity and Magnetism course (MI-EM) because representation use is a central design feature of the curricula and an important learning goal for the course [26, 108]. The MI-EM course is the second of a two-course, calculus-based, introductory physics sequence, which is focused on helping students to develop, test, apply, and refine the basic models in physics. By setting this study in the second semester of the course sequence, we can examine how students' representation choices vary based on questions from the mechanics and EM contexts.

The University Modeling Instruction courses - Modeling Instruction - Mechanics (MI-Mech) and MI-EM - are highly-reformed, active-learning classrooms that combine lecture, laboratory, and recitation (6 hours of class time per week) to create and apply models [26]. In a typical class period, students work through guided-inquiry activities, often using lab equipment, in small groups ( 3 students) that step students through a model building cycle $[26,34,35]$. At the end of each activity, students 
are asked to write down on a small whiteboard (3' x 4') the following: 1) what they learned in the activity, 2) what rules or patterns they can make from what they did, and 3) what they still have questions about. (If the activity asked them to make a model of a phenomena, students may also have their model on their whiteboard.) After creating these whiteboards in their small groups, approximately 8 small groups come together for a large group discussion (referred to as a "board meeting") of what they learned in their groups. Rather than lecturing, the role of the instructor is to facilitate this discussion as well as help guide students through the activity when they are working in their small groups. Ideally, the board meeting is student-led and offers students a place to compare their work, come to consensus on the ideas they were discussing in their activity, and ask questions of one another.

In the Modeling Instruction framework $[28,50,32,26]$, a model is defined as a coordinated set of representations with the ability to explain, predict, and describe a certain set of phenomena $[2,108,1]$. Thus, when asked to make a model, students are expected to create a set of representations that can not only predict what will happen (at a future time or at a different location), but that fully describe the prominent features of a phenomenon and have explanatory power as to why the system might change in the way that it does. For example, when asked to create a model of a positive test charge $(\mathrm{P})$ placed near two positive charges $\left(q_{1}\right.$ and $\left.q_{2}\right)$, we would expect a solution like the one shown in Figure 16, which includes not only equations, but also a system schema, electric potential lines, electric field lines, a force diagram, energy pie charts, acceleration and velocity graphs, an explicit statement about the assumptions the students have made, and a word description of what is happening to the charge. 


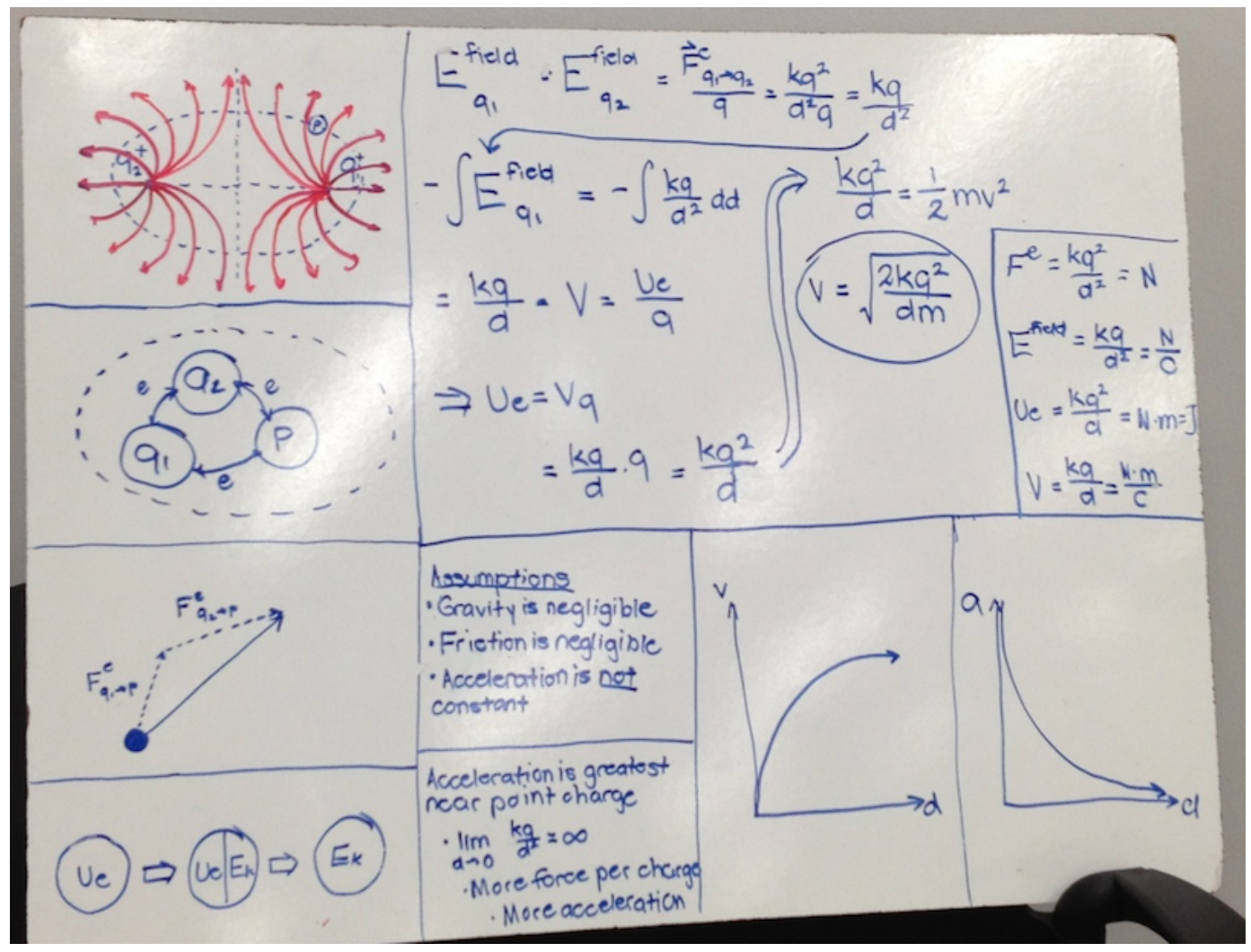

Figure 16: Example of student's model in EM.

This is an example of a conceptual model created by MI-EM students for a point charge (p) placed near two positive charges. It shows how a conceptual model consists of a coordinated set of representations including: equations, graphs, field lines, potential lines, a system schema, a force diagram, energy pie charts, assumptions and a word description. 
As mentioned before, the MI activities are designed to follow a model building cycle, which helps guides students in the construction of each model. Brewe outlined this process as: 1) introduction and representation of a phenomenon, 2) coordination of representations, 3) application of the model to a variety of contexts, 4) abstraction and generalization of the model, and 5) refinement of the model [26]. The first two steps of this process are explicitly focused on helping students to figure out how to represent a particular aspect of a phenomenon and how to coordinate the new representations with those that students already know. Within the curriculum materials, this cycle repeats roughly every two weeks and is used as a means to expand and incorporate new concepts into the model that students are constructing. At the end of the class period, students are also given a homework assignment that asks students to "make a model" of a particular phenomenon that is relevant to what was covered in class. As this process is rather involved and requires creating multiple representations, these assignments focus on one "make a model" question rather than assigning many short problem that ask students to solve for a particular quantity. Thus, students have instruction regarding building, using, and coordinating representations in physics, which is returned to every time that the cycle repeats in class. Furthermore, students have multiple opportunities to practice creating models (and thus using and coordinating representations) both through the in-class activities and the assigned homework.

\subsection{Research questions}

As representation use is a key feature and learning goal of the MI courses, they provide an ideal setting to study students' representation choices. By the end of MI-EM 
course, students have had extensive practice and instruction on using, building and coordinating multiple representations. Thus, we ask the following research questions: 1) By the end of MI-EM, what representations do Modeling Instruction students see as useful together when problem solving? 2) What differences do we see between students' concurrent representation choices on questions in mechanics and EM contexts?

\subsection{Data Collection}

To be able to answer these research questions, we designed a survey called the Problem Solving and Representation Use Survey (PSRUS), which consists of 25 physics problems from across the introductory physics content. Based off of a modified cardsort survey, the PSRUS was designed as a paper survey, where twelve of the questions are focused on a mechanics context, while thirteen of the questions focus on EM mate-

rial. For each question on the PSRUS, students were asked to list the representations that they would use to solve that problem - they did not have to actually solve the problem.

To aid in this process, students were also given a list of the common representations used in the MI-Mech and MI-EM courses (shown in Figure 17), which included: graphs, equations, words, assumptions, motion map, picture, system schema, free body diagrams, momentum vectors, energy pie charts, energy bar charts, circuit diagrams, right hand rules, potential lines, and field lines. The list of representations also included an "other" option, so students could write in a representation if it wasn't already listed. There was no limit to the number of representations that students could choose. In theory, students could list anywhere from one to sixteen representations for each question; however, all of the representations are not necessarily applicable 
to all of the questions. For example, if a student listed a circuit diagram as a representation on a question about projectile motion, this would indicate that the student was not taking the survey seriously and would invalidate their survey.

Beyond checking for reasonable responses to the survey, we were not interested in grading this survey or looking for the "right" representations for a problem based off expert responses. Several researchers have shown that experts and notices use representations and approach problem-solving in different ways $[6,19,20]$. If we were to compare student responses to experts', this would ignore the representations that students may find useful (but experts skip over) as well as introduce implicit bias into the study depending on who the experts were [72]. Instead, we examine the choices that students would make as they are. Since we were not grading the survey, we used a test panel consisting of faculty, graduate students, and undergraduate students at Florida International University to address the the validity, reliability, and fairness of the survey $[96,109,71]$. Based on feedback from the test panel, the survey was edited before it was given in the classroom. Further discussion of the validity, reliability, and fairness of the PSRUS is given in the appendices.

The data presented in the subsequent sections are from the PSRUS given at the end of the Spring 2016 semester in the MI-EM course at FIU. Between the Spring 2015 and Spring 2016 semesters, there was a significant reform of the MI-EM materials, which was motivated by the success of the MI-Mech course on multiple measures $[38,41,69,44,90]$. The primary purpose of the course reform was to align the MIEM curriculum with the Modeling Instruction framework, in particular to align the materials with modeling cycle outlined in the previous sections [108]. By the end of MI-EM (after the curricular changes), students had covered the topics of electricity, 


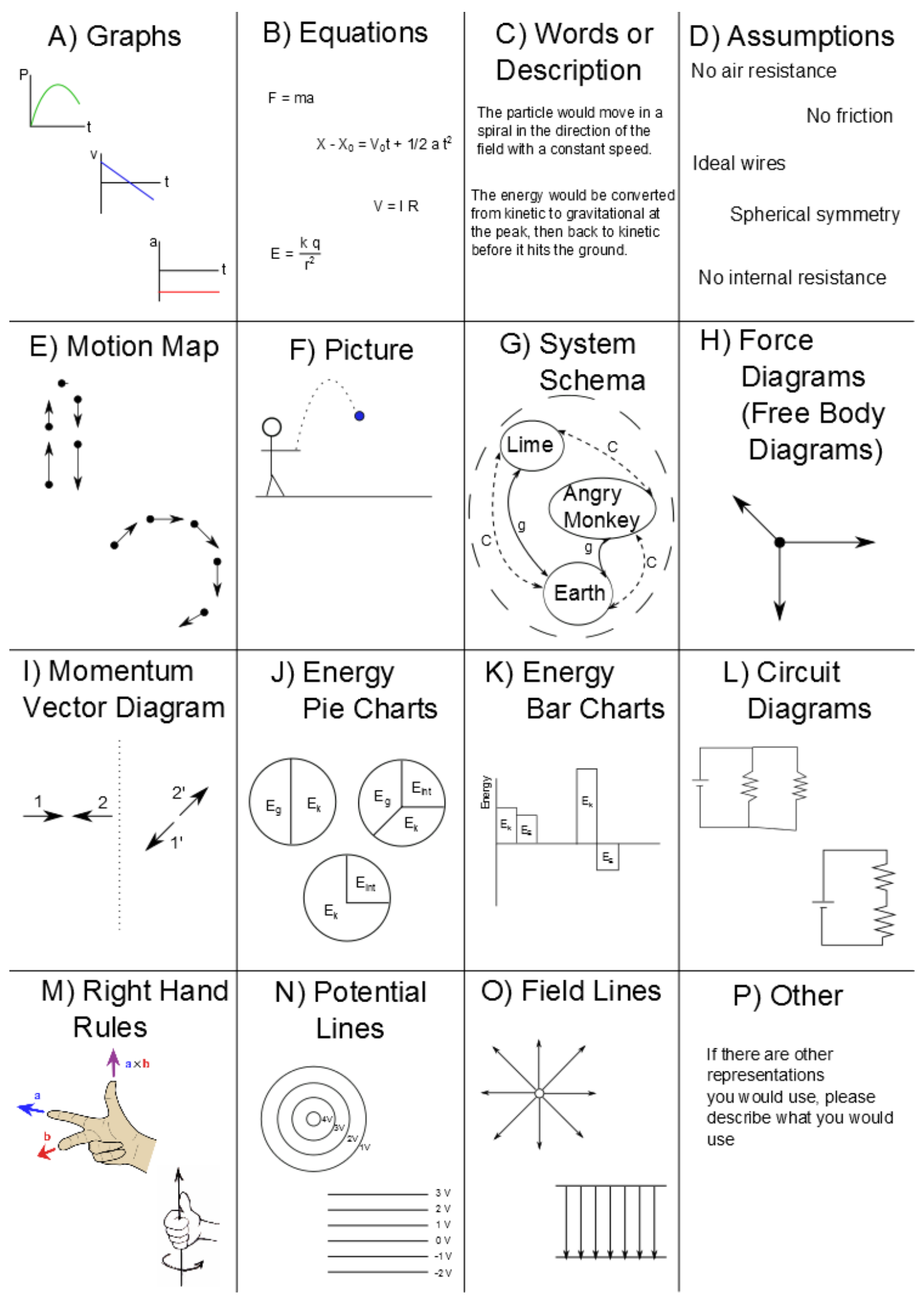

Figure 17: Representations given on the PSRUS.

This figure shows the list of representations that were given to students along with the Problem Solving and Representation Use Survey (PSRUS). 
circuits, magnetism, and optics, in addition to the topics covered in the mechanics pre-requisite course.

In the Spring 2016 semester, two sections of MI-EM were taught in a 75-student format, with students who were representative of FIU's student population (61\% of the student body identifying as Hispanic, 15\% as White Non-Hispanic, 13\% as Black, 4\% Asian or Pacific Islander, and 7\% as other minority groups) [98]. Based on major requirements, the two sections were primarily (though not exclusively) composed of engineering and life-science majors. For each section, we gave the PSRUS in the penultimate week of the course. Of the two sections, one was taught by a physics faculty member (who had taught MI before), a graduate student (who had taught MI before and led the curriculum reform project), and two undergraduate learning assistants (LAs) who had take MI-EM the previous year. The other section was taught by a postdoctoral researcher (who was new to MI), two graduate students (one of whom had taught MI before, one of whom was new to MI), and three undergraduate LAs (two of whom where new to MI but had served as LA for other courses). The two sections used all of the same materials for the course, had a shared prep meeting, developed common exams, and shared grading rubrics across the two sections. When we compared students responses from each section on the PSRUS, we found no difference in the number or variety of representations that students chose; thus, we combined the responses for the two sections into a single data set for the purposes of our analysis. In total, we had valid surveys from 121 students (51 Female/70 Male) across the two sections on the Post-PSRUS, which are the data used in the analysis described in the subsequent section. 


\subsection{Network Analysis}

Since students are free to respond to any question with 1-16 representation choices, we used a network out of student responses to capture what representations students are choosing concurrently on the problems in the PSRUS. Network analysis has been widely used and developed outside of physics to address a wide variety of topics from traffic flow in cities [110] and flavor profiles in food around the world [111]; however, network analysis has recently been used in Physics Education Research (PER) to look at student interactions (Social Network Analysis) [112, 113, 44, 114] or patterns in student responses on conceptual surveys [115].

At it's most basic description, network analysis is a powerful tool to describe what are the "things" and how are the "things" related. In network terms, the "things" are called nodes and how they are related is represented by an edge (or a line connecting two nodes). In the example of social network analysis in PER [116], the nodes are typically representing students, and the edges are showing which students interacted in class. To answer our research question of what representations students choose on the same problem, we will be building a network where the nodes are the representations (shown in Figure 17) and the edges show when a student has said they would use those representations together on a question of the PSRUS. The following sections outline in more detail both how we built and simplified this network for the following analysis.

\subsubsection{Building the Network}

To build the network, we started with the student responses to each question (shown in Figure 18a). For example, if a student says they would use equations (eq), graphs 
(gr), and a picture (pic) in response to the first question, each of these representations would be connected in the network - shown in Figure 18b. Thus, in this network, the representations (equations, graphs, and pictures) are the "things" and become the nodes in the network, which are related by when a student chooses them together on a question. This means that each edge represents a student's response on a single question.

If we move to the second question, a student could choose equations (Eq), a system schema (SS), energy pie charts (Pies), and a force diagram (FD) as their response. Then for the second question, equations, system schema, energy pie charts, and force diagrams are all connected with edges - shown in Figure 18c. The edges from Question 2 are shown in gold to distinguish them from the edges that are in the network from the first question (shown as red edges). Likewise, if a student responds that they would use a picture (pic), force diagram (FD), and an equation (eq) on the third question, these representations would again be connected - shown as the purple edges in Figure 18d. There are now two lines connecting equations and force diagrams (as a pair) and connecting equations and pictures (as a pair). As the network is built the number of repeating edges is called "edge weight." In terms of this network, the edge weight represents the number of times that students listed the pair of representations together on a question. In this example with the three questions, we would ultimately mark each edge and denote the weight of each edge (shown in Figure 18e).

By following this process, we can build a network for a student's responses to the mechanics questions and a separate network for their responses to the EM questions. Since the nodes in each student's network are the same (the 16 representations), we can also combine the networks across students. In doing this process, we ultimately 


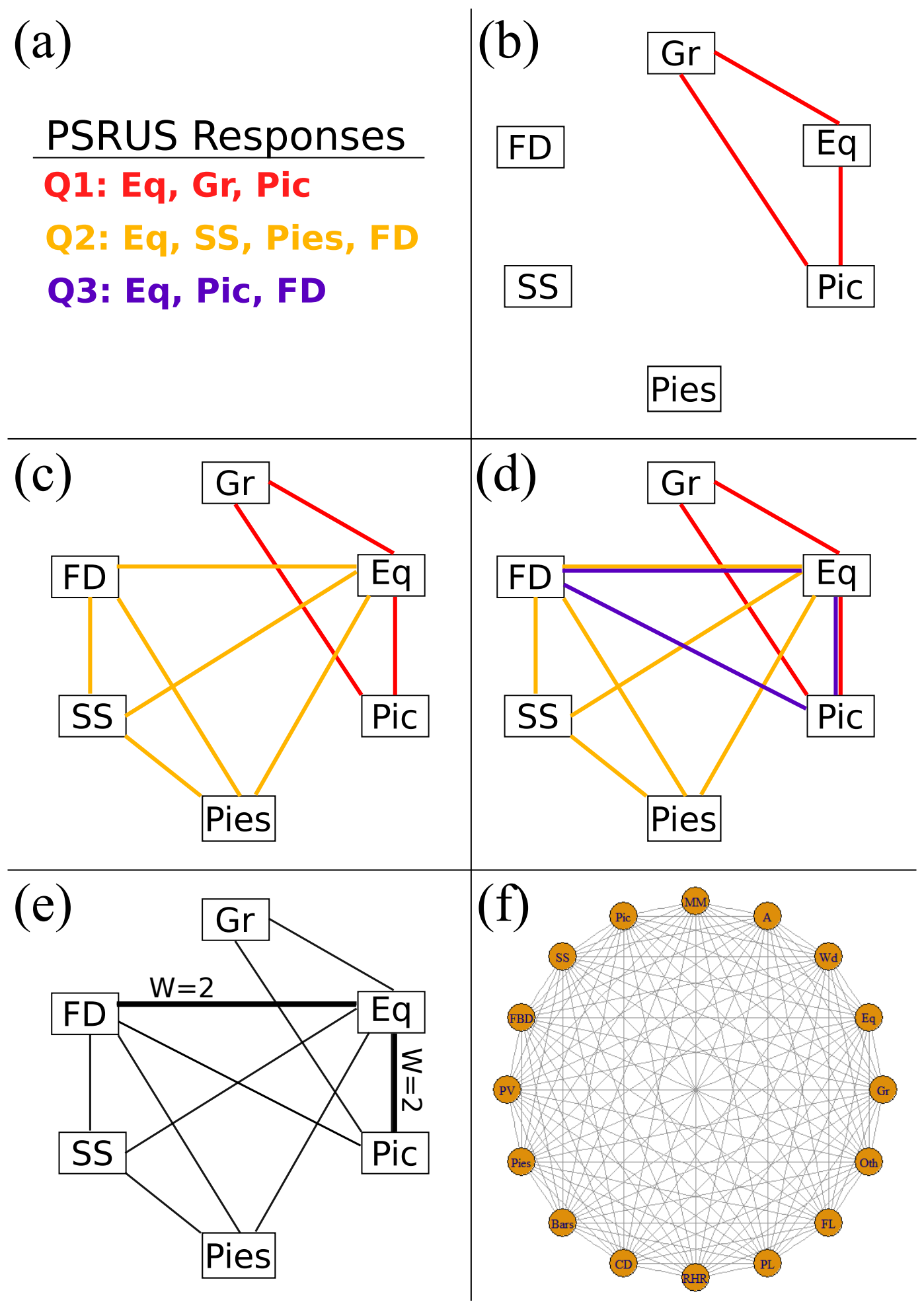

Figure 18: Process for building the representation networks.

This figure shows how the network was constructed from examples of student responses on the PSRUS. (a) shows the example responses given on three questions of the PSRUS. (b) shows how the representations for Q1 would be connected in the network. (c) and (d) show the network construction for Q2 and Q3 respectively. (e) shows the network for all three question 1 ducluding the weighted edges (darker lines labeled as $W=2$ ). (f) shows the full network for the responses from all students on all of the EM questions. 
built two networks of all students' responses to the PSRUS: one network for the mechanics questions and one network for the EM questions. As an example of such a network, the entire EM network (built from all 121 PSRUS responses to all 13 EM questions) is shown in Figure $18 \mathrm{f}$.

In each of these networks, they are built on multiple students' responses and representation choices. This means that in interpreting the network results, we are only able to answer course-level questions and show how the class as a whole chooses representations. These networks do not reflect each individual student's responses or which particular question that response was for. Instead, these networks show the class-wide representation choices to the "average" mechanics or "average" EM question.

Perhaps the most relevant feature then of the EM network (shown in Figure 18f) and the mechanics network is that they are both highly-dense networks. Over the 121 responses to 13 questions almost all of the individual pairs of representations were chosen at some point. These networks are also weighted and an undirected, meaning that the number of times a pair of representations is chosen on any question is stored as the weight attribute of that edge and it is marked as line (there are no directional arrows in this network). It is worth noting that we could have alternatively built the network using the students as the nodes, with connections between students showing if they used the same representation on the same question. This means that the network we have constructed in Figure 18 is a projection of a larger bipartite network built from the PSRUS results; however, we choose to examine only the network where the representations are the nodes since this is the network that would allow us to examine concurrent representation choices and address the research questions outlined above. 
While the network shown in Figure 18f accurately reflects the representation choices of the whole class, it is not particularly useful in this form. It is extremely difficult to "see" the representations that are important to student's problem solving and it is impossible to tell what are the representations that are repeatedly used together by students. Thus, a filter is warranted to sparsify these networks.

\subsubsection{Network Filter}

In a highly-dense network, a filter is used to reduce the number of edges (and sometimes nodes) to highlight which edges/nodes are important to the network. Depending on the choice of filter, both how "important" is defined and how the filter removes edges can vary widely.

One such filter is simply to apply a threshold to the weighted edges, keeping only the edges that have a large weight and removing the edges that are smaller than the threshold. While effective and easy to implement, this threshold filter will only emphasize large-grain results and systematically ignores local patterns amongst the nodes [117]. There is a similar effect in course-graining filters, which typically use a "minimum spanning tree" (or dendrogram) to combine nodes and edges. These kinds of course-graining filters serve to "zoom out" of the network, but lose important information about what the individual nodes are and those connections [117].

Instead, we chose to use a disparity filter [118] for this analysis, which was designed specifically for weighted, undirected networks. The disparity filter works by examining the edges for each individual node, rather than considering all of the edges in the network at once. For each node, the strength of that node is calculated by summing the weights of all the edges connected to that node and is used to normalize the 
weights of the edges for each node. The purpose of the disparity filter is then to retain the edges that contribute the most to a particular node's strength. By filtering the edges on a per node basis, the disparity filter retains the edges that are locally important for that node.

In contrast to the other filtering methods, the disparity filter also compares the proportional strength of each edge to a null distribution [118], which removes edges from the network that do not meet a predefined threshold (alpha). By using this method on a per node basis, this means that the final filtered network "not only contains the links [edges] carrying the largest weight in the network, but also all links [edges] that can be considered, according to a predefined statistical significance level, to define the relevant structure (signal) generated by the weight and strength assignment" [118]. For this analysis, we treat the threshold as a probabilisticallymotivated adaptive threshold, rather than as a statistical test [119].

To set the threshold for this analysis, we chose an alpha level of 0.2 . As there is not a conventional alpha level in network analysis (unlike the typical $\alpha=0.05$ value established for parametric test comparisons), we chose $\alpha=0.2$ by picking an $\alpha$ that maximized the number of nodes, minimized the number of edges, and maximized the total strength of edges represented in the network. This is within the optimal range of 0.01-0.5 outlined by Serrano, Boguñá, and Vespignani.

\subsubsection{Edge Betweenness Centrality}

The disparity filter described in the previous section reduces the overwhelming number of edges to those that are important for each node and statistically different from a random network - answering the question of which edges are important in the net- 
work. It also removes the nodes that do not have any important edges; however, it cannot tell us which of the remaining nodes are important.

As with the filter and choosing the important edges, the "importance" of nodes can be determined by many different measures - one of which is called node centrality. Node centrality broadly looks at which nodes are important within the network by looking at the number of connections (degree centrality [120]), looking at the weighted edges of a node's nearest neighbors (page rank centrality or eigenvector centrality[121]), or looking at the shortest paths between nodes (edge betweenness centrality [122]).

For this analysis, we choose to use edge betweenness centrality as the measure of "important" nodes. A node with a high edge betweenness has a high probability of existing on the shortest path (or geodesic path) between any two nodes. In terms of our network, where the nodes are representations, this means that a representation with a high edge betweenness serves as a "high connector" representation that would help students get from one representation to another via the shortest path.

Thus, we use both a disparity filter and edge betweenness centrality to reduce the highly-dense network to that which includes only the important edges and nodes.

\subsection{Results}

After applying the disparity filter to the EM network (from Figure 18f), we get the reduced networks shown in Figure 19, where Figure 19a shows the mechanics network and Figure 19b shows the EM network. In each network, we have used the following abbreviations for the representations: Gr - graphs, Eq - equations, Wd - words, A - assumptions, MM - motion map, Pic - picture, SS - system schema, FBD - free 


\section{(a) Mechanics}

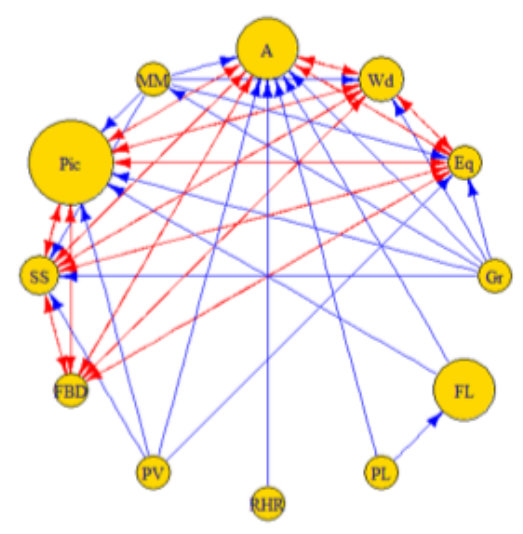

(b) EM

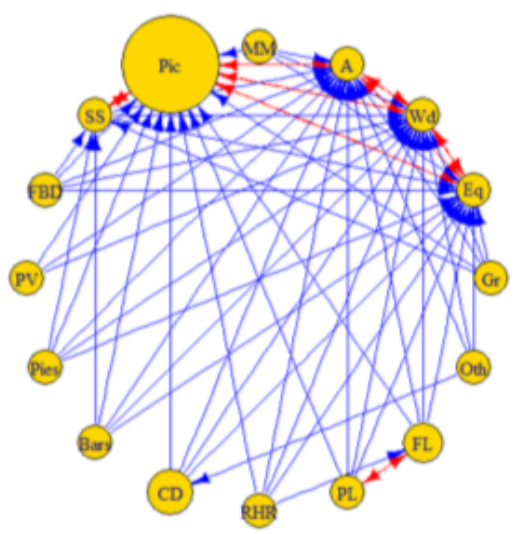

Figure 19: Filtered networks for mechanics and EM.

This figure shows the filtered networks for the post-PSRUS in the Spring 2016 semester. (a) shows the filtered network for the mechanics questions only. (b) shows the filtered network for the EM questions only. Between the two networks is the list of representation abbreviations used in both networks.

body diagram, PV - momentum vectors, Pies - energy pie charts, Bars - energy bar charts, CD - Circuit Diagrams, RHR - Right Hand Rules, PL - potential lines, FL - field lines, and Oth - other.

Both of these networks are now directed as it possible for an edge to be significant for one of the nodes in the pair or for both, which are shown as blue, singleheaded arrows and red, double-headed arrows respectively. For example, if a blue one-directional arrow points from graphs to equations this would mean that graphequations edge held a significant amount of the strength for graph node but not for the equations node. In other words, if students said they would use graphs they likely would have also listed equations, but if they listed equations, they did not necessarily list graphs. The size of each node in the network is scaled with the edge betweenness of each node - the larger the node, the more geodesic paths use that representation 
to get from one representation to another. Thus, the larger the node, the larger role that representation serves as a "connector" between other representations.

From the comparison of Figures 19a \& 19b, there are several features that immediately stand out. First, there are more double-sided (red arrows) in the mechanics network than in the EM network. This tells us that there are a central set of representations that students consistently choose on mechanics questions: picture, words, assumptions, equations, system schema, and force diagrams (or free body diagrams). This is not only apparent from the large number of red arrows, but also by the fact that each of these representations in the central set is connected to the others in the set by a double-headed arrow (each representation in this set has 5 red, double-headed arrows). When compared to the double-headed arrows in the EM network, there are fewer double-headed arrows with the set of fully connected representations reduced to only four: pictures, assumptions, words, and equations. The system schema is repeatedly chosen with pictures but is not consistently chosen with the other representations in this set, and the only other double headed arrow shows the relationship between field and potential lines. This result implies that there is a common "core" of representations in mechanics that students rely on in their problem solving that is reduced when moving to an EM context.

Between the two contexts, the "core" representations that students continue to see as applicable are the verbal representations (words and assumptions), a pictorial representation (pictures), and a mathematical representation (equations). This implies that the more diagrammatic representations (such a force diagrams, energy pie charts, or motion maps) vary more widely both with and between the mechanics and EM contexts. 
Figure 19 also shows that there are more representations in EM that students are choosing when compared to Mechanics as evidenced by all 16 representations from the survey represented as nodes in the network. In contrast, the mechanics network only has 12 nodes - with the missing representation being: energy pie charts, energy bar charts, other, and circuit diagrams. (The representations typically introduced in EM - such as right hand rules, field and potential lines - that are present in the Mechanics network could perhaps indicate students relating ideas of cross-products and fields to torques and gravitational contexts, but more likely are a result of statistical variance in the data.)

Of the representations that are missing, the "other" representation and "circuit diagrams" are understandably not shown in the mechanics network; however, the fact that the energy representations are not present in the mechanics network may suggest that students do not see these as related to the other representations they use when problem solving. Again, this does not mean zero students chose energy representations in their mechanics problem solving, but instead that it was not prevalent enough in the responses across the course to be significantly different from a randomized network. The lack of energy representations in the mechanics network could be an artifact of the time of this test - it was given at the end of the MI-EM course; however, even then, this result is indicating that students may not remember or view energy as a useful approach to many problems. This is especially concerning as the Modeling Instruction curriculum was designed with an "energy-first" order to the content to emphasize the role of energy in mechanics contexts [13]; in addition, many activities in the curricula (both in MI-Mech and MI-EM) are designed to coordinate these energy 
representations with the other representations used in the course - like system schema and equations $[26,13,8]$.

Relatedly, there are also more blue, one-directional arrows overall in the EM network compared to the mechanics network. This effect may be due in part to the larger number of representations or nodes; however, there are also a larger number of one-directional arrows per node. This indicates that students use a wider variety of representations on EM problems. Rather than students consistently using the same pair of representations (which would be represented by a single edge containing the majority of the strength for a given node), there are instead multiple edges (or multiple pairs of representations) that carry a significant amount of the strength for that node. This could be interpreted as students do not have a consistent model that they use in EM (unlike in mechanics), which is supported by the difference in the number of red, two-directional arrows.

Finally, when we compare the size of the nodes in the two networks, in EM there is really only one representation that has a large edge betweenness; whereas in mechanics there are several representations that serve as these large "connector" representations. This indicates that there are multiple pathways among students' choices in mechanics that could help them easily get from one representation to another. However, in EM there is a far more restricted choice in getting from one representation to another easily. Since edge betweenness is based on a measure of the shortest path between representations in this case, this tells us that students are more aware of how the different representations are related in a mechanics context, but that these relationships are not as apparent in their EM choices. 
In both mechanics and EM contexts, the representation with the largest edge betweenness is the "picture" representation. This means that the shortest path between any two representations in either network likely goes through a picture and that if you have a picture representation it is relatively easy to get to any of the other representations. This high edge betweenness could be a result of the suggestion to "first draw a picture" that students repeatedly hear in science classes. Alternatively, the high edge betweenness of pictures may suggest the importance of a picture as a representation for students' problem solving [63], especially as a means of relating to other relevant representations. Particularly in EM contexts, the results of Figure 19 suggest the picture is especially important as a "connecting" representation since there are no other representations with a high edge betweenness to facilitate the relationships between representations.

\subsection{Conclusions}

In this paper, we used network analysis to address what representations MI-EM students see as useful together in their problem-solving and to compare their responses in both mechanics and EM contexts. After building and filtering the networks from the post-PSRUS survey results, we found that there are visible differences between students' concurrent representation choices in mechanics and in EM shown in Figure 19. Since there are a set of representations in mechanics that are fully connected by bidirectional arrows, we conclude that there is a common set of representations that students frequently use together in Mechanics contexts. In comparison, the EM network has far fewer bidirectional arrows but an far larger number of unidirectional arrows. This indicates that students rely on a wider variety of representations in EM 
and do not repeatedly use the same set. We also see that there are more representations (or nodes) in the EM network, which given the new content is not surprising. However, the fact that the energy representations like energy pie charts did not have any edges that were significantly different from the randomized network is concerning, especially for mechanics questions. This could indicate that students do not see energy as a useful part of their problem solving on these problems or that they do not see energy representations as connected to the other representations that they would rely on. Finally, we see that pictures serve an especially important role in students' problem solving. In both the mechanics and EM networks, pictures has the highest edge betweenness, meaning it functions as the largest "connector" between representations.

These results highlight important implications for teaching and curriculum design in introductory physics, specifically for the MI sequence and perhaps for other courses at large. First, a picture can serve an important role in helping students to coordinate representations, especially in EM as there are few other representations that serve as high "connector" representations. This ties back to Ainsworth's work on using multiple representations to help students bridge from familiar representations to those are more abstract $[5,10]$. Our work suggests that the role pictures play a critical role in connecting the representations. Furthermore, students may need more help recognizing when representations are important or relevant in EM. There are many more representations available in EM and students are using a wide variety of representations rather than consistently using the same set over and over. As they move away from the common "core" of representations in mechanics, making sure students know why those representations are not appropriate anymore or why they 
need to rely on new representations would be important scaffolding to build into a course. Finally, our results indicate that even more work may need to be done in the MI sequence to emphasize the role of energy in problem solving and how those connect to the other representations, particularly in the EM semester. returning to the idea of energy and why it may (or may not) be useful in a context may serve as a reminder to students that energy is often a valid (and easier) solution path.

In a broader sense, this study is an example of extending network analysis techniques to classroom data that move beyond social networks in the classroom. We argue that in using network analysis and this survey, we have a lens into what representations students are seeing as useful in their problem solving in general, and specifically what representations students use and coordinate together, that we would not see otherwise. This analysis has shown many of the patterns in students' representation choices and is aimed at understanding an aspect of their problem solving approach, as opposed to examining students' content understanding. In recent years, there has been a push in Physics Education Research to focus on problem solving as skill, and we have shown in this paper that network analysis may serve as a useful tool to understand students' problem solving approach.

This study has several limitations, based on the survey design, how we built the networks, and our student population; however, we aver that these limitations also open up new research questions and avenues for future work. Our survey only asked students to list the representations that they would use on a given problem, not to actually solve the problem or the order in which they would use the representations. This means that our results must be interpreted as what representations students would see as useful and not as the representations that students used to solve these 
problem. A similar network could have been constructed based on examples of students' solved work (i.e. an exam problem or a homework problem). Likewise, we could construct a directed network based on the order that students use their representations, which could answer questions about students' problem solving process and perhaps even the flow of information through such a network. We also treated all of the listed representations as equal in this analysis, meaning we did not distinguish between perhaps more general representations (like words or pictures) and context specific representations (like circuit diagrams or momentum vectors). In future work, we could address the generalization of the representations through a cluster analysis of the representation networks.

The PSRUS was also designed specifically to look at students' representation choices in Modeling Instruction courses. There are several representations, such as the system schema and energy pie charts, that are heavily emphasized in MI-Mech and MI-EM that are not emphasized (or perhaps not even present) in other introductory physics courses. If this survey were to be given in other classes, substantial edits may be needed to assure that the survey is valid and reliable in those new contexts. This would be indicated to answer questions like how do students' representation choices in MI compare to those in the equivalent lecture/laboratory sections.

As a part of the design for the MI courses, the questions on the PSRUS were developed to cover the range of content presented in MI-Mech and MI-EM courses, with at least two questions from each of the basic models explored in each MI course. One can imagine that the networks might change significantly if different questions (or proportions of questions) were asked. For example, if the majority of questions 
on EM portion of the survey were about circuits and their applications, we would imagine that circuit diagrams might play a more important role in the EM network.

Finally, we believe that there are many interesting questions to ask regarding the comparison of students' representation networks in the future. For example, this survey could be used to examine the progression of students' representation choices over a single semester or even the two-semester sequence of MI courses. We could also compare the representation choices of students individually (rather than constructing the network from the whole class). With individual student networks, it would be possible to correlate or possibly predict student outcomes (such as grades) with their individual network measures or problem solving approach (such as degree or betweenness centrality). As a future direction of this work, correlating these network measures with student outcomes could build on the studies that investigate if multiple representations help students in learning physics. If conducted at FIU (as a Hispanicserving institution), such a study could contribute to the body of literature on how to support underrepresented groups in physics.

The analysis presented in this dissertation is only scratching the surface of the tools available through network analysis. Some network tools, such as community detection or cluster analysis, show promise for finding the subset of representations that students concurrently use or perhaps even showing what are the representations that students see as complementary or overlapping. We hope to address some of these questions in our future work. 


\section{CONCLUSION}

Because representations serve a critical role in science and in physics specifically, this dissertation is focused on studying students' representation choices in the Modeling Instruction - Electricity and Magnetism (MI-EM) classroom. In MI-EM, representation use and coordination is an explicit learning goal, with activities and instruction centered on representation use as a part of the model building cycle [26]. As MI-EM is the second semester in the two-course introductory physics sequence, it provides then an important opportunity to study how students use representations across the introductory content, from mechanics to electricity and magnetism.

Thus, this dissertation set out to answer the following research questions. (1) Prior to the curriculum changes, what representations do MI-EM students rely on in introductory physics contexts (both EM and mechanics)? Additionally, how does the MI-EM course affect the number and variety of representations that students choose for both students who took MI-Mech (returning to the MI course format) and those who did not (new to the MI course format)? (2) How do changes in the MI-EM curriculum between the Spring 2015 and Spring 2016 semesters impact the number and variety of students' representation choices? How do these vary for new and returning students in both mechanics and EM contexts? (3) By the end of MI-EM (after the curricular changes), what representations do students see as coordinated and useful together in the problem solving over mechanics and EM contexts?

To answer the first research question (as described in Chapter 2), I developed the Problem Solving and Representation Use Survey (PSRUS), which was given at the beginning and end of the Spring 2015 MI-EM course. By using non-parametric statical 
tests (Mann-Whitney-Wilcoxon test, Wilcoxon-Signed-Rank tests, and Cliff's Delta Effect Sizes), I found that students who were returning to MI-EM from the MI-Mech course use more representations and a wider variety of representations than those who took the lecture-format mechanics course. However, over the course of MI-EM, students who were new to the MI environment in MI-EM have significant gains in their representation choices in both EM and mechanics contexts. This means that the new students are transferring representations to contexts that were not covered in the MI-EM course, indicating that the MI-EM course is impacting more than students' content learning and providing more tools for these students in their problem solving. In contrast, returning students' representation use stays more or less constant. I also found that there is still a separation in the number and variety of representations that new and returning students use by the end of the semester, particularly in Mechanics contexts. This indicated an inequity in the course and that students are leaving the class with a different set of experiences and skills depending on what type of mechanics course they took. These results motivated changes made to the MI-EM curriculum to incorporate new students into the MI classroom as a part of the larger modifications made to the MI-EM curriculum between the Spring 2015 and Spring 2016 semesters.

These changes in the MI-EM curriculum motivated our second research question, as addressed in Chapter 3. Using the PSRUS results across both the Spring 2015 and Spring 2016 semesters, I found that students (both new and returning to MI) used more representations across mechanics and EM questions through Mann-WhitneyWilcoxon tests, Wilcoxon-Signed-Rank tests, and Cliff's Delta Effect Sizes. When I examined what representations students were using differently in the Spring 2016 
semester (compared to the Spring 2015 semester), I found that students chose word descriptions, pictures, assumptions, potential lines, and right hand rules significantly more in the Spring 2016 semester. Since studies have shown that students who use more representations are more likely to be successful in their problem solving, the MI framework incorporated explicit instruction and practice with these representational tools. As the MI-EM curriculum was designed to align with this framework, this increase in representation choices is interpreted as a positive outcome of the curriculum modifications. In addition, I found that the significant difference between new and returning students' representation choices disappear in the Spring 2016 semester. This implies that by the end of the Spring 2016 semester, students are leaving the course with a similar representational tool set and that explicit design can help students reach an even level (in terms of representation choices) in new classroom formats like MI-EM.

Finally, I address the third research question (in Chapter 4), using network analysis to examine the representations that students concurrently use on the mechanics and EM questions of the PSRUS. I found that on mechanics questions there is a consistent "core" of representations that students repeatedly choose together, whereas in EM, this "core" is much smaller and disconnected. Instead, students use a wider variety of representations across the questions in EM. Perhaps not surprisingly, there are more representations overall in the EM course; however, the lack of energy representations in the mechanics networks indicates that students may not see energy as a viable tool in their problem solving. In both the mechanics and EM networks, I found that pictures had the highest edge betweenness, which may indicate that pictures serve a large role in connecting the different representations together. This 
is particularly true in EM contexts, where there are no other nodes with high edge betweenness.

Together, these papers examine the representation choices of students in MI-EM and answer the research questions outlined previously. For instructors, curriculum developers, and researchers, this work indicates that if representation use is a goal, explicit instruction and time must be spent working with these representations.

\subsection{Implications and Intellectual Merit}

More broadly, this dissertation adds to the field of Physics Education Research by exemplifying the evaluation of a curriculum on learning goals of a course that move beyond content learning. Through the building, testing, applying and refining of models, one of the goals of MI is to improve students' use and coordination of representations [26]. Previous measures of the MI curriculum, like conceptual surveys or affective surveys $[38,41]$, have not been able to address this aspect of the course. If this is a learning goal of the course, then part of the evaluation of this curriculum should address this learning goal. In the results from the PSRUS survey, important information about students' representational choices was learned that would not have been found by other means. For example, the fact that the MI-EM course impacts not only students' representational choices in EM content but also in mechanics shows that MI-EM is impacting students' problem solving strategies. This shows improvement in a skill over the course of the semester that would not have been seen through other measures.

Furthermore, I have shown that changes to an introductory curriculum can be aimed at and impact more than students' content knowledge. Paying attention to the 
problem solving skills (in addition to content learning) is just as important, especially as these are part of the skills that employers expect of their future employees to have [123]. Representation use and coordination is an important aspect of problem solving and can serve as a means to evaluate the impact of curricular changes on students' problem solving rather than measuring the impact on scores. Thus, I encourage other instructors and researchers to consider the learning goals for a course as well as the assessment of a course that move beyond content, particularly those that are skill-based.

In addition, this work has highlighted important differences between students in the MI-EM course depending on the type of mechanics course that they took. Particularly in the courses that are highly reformed with different kinds of learning goals (i.e., creating models or coordinating representations), it is easy to begin the second semester course with where the first semester left off, building off of the structures, ideas, practices, and culture introduced in the first semester course. However, I argue that careful attention needs to be paid to incorporating students who did not take the reformed version of the prerequisite course. In the case of MI-EM, there were significant differences in the representations that new and returning students rely on particularly in the beginning of the semester and modifications to the MI-EM curriculum contributed to increasing new students' representation use to the same level as their counterparts who where returning from MI-Mech. For instructors, curriculum developers, and researchers, this work suggests that attention to how students experiences might differ based on their prerequisite experiences is needed and that modifications to curricular materials may help address inequities in skills such as representation use. 
Finally, I have introduced an application of network analysis to classroom data that uses students' representation choices rather than examining social interactions in the classroom. By using network analysis, I was able to identify patterns in students' representation choices, particularly in their concurrent representation choices that would have been difficult to see otherwise. Since network analysis is a powerful tool for importance of and relationships between different components, I would encourage other researchers to consider the other kinds of relationships in the classroom that may impact students' experiences and learning, perhaps including but not limited to student interactions.

\subsection{Limitations of the PSRUS}

The first and foremost limitation of the PSRUS is that I only asked students to list the representations that they would use on these problems. This means that I am measuring the number of representations that students think they will need, not what they actually use to solve each question. From the standpoint taken in this dissertation of representations as tools in problem solving, I argue that studying the representations students think will be useful is informative in itself as it highlights the tools that students would draw on in their problem solving.

From this same viewpoint of representations as tools, increasing the number of representations that students see as useful and coordinated serves to increase the likelihood of success in problem solving $[7,49]$ and facilitates deeper conceptual understanding [5]. The MI courses (both MI-Mech and MI-EM) are, in part, designed with representation use and coordination as an explicit learning goal. Use of multiple representations features heavily in the curriculum materials, in student assessment 
(on homework, formal labs, and exams), and in the day-to-day activities of the class. Thus, I argue that a positive outcome particularly for this class is an increase in representations over the course of the semester. As a result, the work in this dissertation has focused on the differences in the number, variety, and co-occurance of the representation choices that MI-EM students make over the course of the semester.

I recognize that an increase in number or variety of representations is not a positive outcome in all contexts. In fact, as expertise is developed, there may be a decrease in the number of representations chosen as experts frequently skip steps or no longer require the external representations as a part of their problem solving process. Instead, Kohl and Finkelstein have shown that rather than using more representations, experts are able to translate between representations more easily [19]. If the PSRUS were to be given in upper-division courses, the interpretation of changes in representation choices would be less clear. Thus, I have limited the scope of the PSRUS (and its interpretation) to the introductory physics classrooms, where such expertise is likely still undeveloped.

Finally, the PSRUS was developed to particularly examine students' representation choices in MI-EM. As a result, there were several representations included on the given sheet with the PSRUS that were developed for the MI courses that we would not expect new students to be familiar with, namely system schema, energy pie charts, and energy bar charts. Therefore, we would expect new students to have a lower average number of representations per question (as shown in Figures 4 and 14) due to their unfamiliarity with several of the listed representations. 


\subsection{Future Directions}

This study was designed to examine students' representation choices, particularly in the MI-EM course, which is an important consideration and limitation of these papers. Since the context of this work is limited to a particular classroom, the results (and their interpretation) are limited to the MI-EM course. One possible future research direction would be to compare students' representational choices across instructional formats to generalize the patterns and trends I found in the work. However, the PSRUS was specifically designed to look at students' representation choices within the MI-EM course. Thus, the PSRUS was developed and all validation work was carried out within the MI environment. Future work will include validation in other instructional environments.

Another possible research direction from this work would be to compare the representation choices that students say they would use to those that they actually use when solving a problem. By design, the PSRUS only asks students to list what representations students would use rather than to completely solve the problem. Thus, these results are all interpreted as students' representational choices. This was done to be able to survey students' ideas of representations across a wide range of physics topics. In order for students to complete this survey in a reasonable amount of time, students could not be expected to actually solve the problems. However, I recognize the limitations of this design. Students may say that they would use representations that they would typically skip over or they may find in actually doing a problem that they need a representation they had not listed. In future work, I hope to compare the representations that students say they would use to those that they actually rely on to solve the problem. 
This work also does not address why students are using different representations in different contexts, how the MI-EM course helps them apply multiple representations, or how students are coordinating these representations. I have shown that there are differences in students' approach to mechanics and EM problems, which also is highly dependent on the type of mechanics course that students took; however, I would need to design a new study to be able to address why students are making these choices and how they view these relationships between representations.

Finally, I believe there are many other interesting questions within the scope of this work using network analysis, especially given that there are a robust set of network analysis techniques from other fields already developed. For example, I could compare how students' representation networks change from the beginning to the end of the semester or the differences between how the new and returning students in MI-EM coordinate representations. In addition, if the PSRUS were modified to ask for the order students would use the representations, network analysis could be used to study the role of representations in students' solution paths or to track the flow of information as students complete problems. Currently, the PSRUS only asks for the types of representations and thus is limited non-directional networks (prior to filtering). Furthermore, I could have examined the alternate projection of the bipartite network for the PSRUS data, which would have shown how the students were related based on their representational choices on the survey. This work is only scratching the surface on what is possible with network analysis on the PSRUS data, and more generally with classroom data. I hope to address some of these questions in our future work. 
Ultimately, the work in this dissertation examines students' representation choices as a means to understand students' problem solving approaches in introductory physics courses. I hope that this work serves as an example to other researchers, curriculum developers, and instructors to evaluate courses on skills-based learning goals (as a part of other measures) and to consider how students are incorporated into a second-semester reformed class, particularly if they took an alternative prerequisite course. 


\section{REFERENCES}

[1] P. S. Oh and S. J. Oh, "What Teachers of Science Need to Know about Models: An overview," International Journal of Science Education, vol. 33, no. 8, pp. 1109-1130, 2011.

[2] S. Manthey and E. Brewe, "Toward university modeling instruction-biology: Adapting curricular frameworks from physics to biology," CBE Life Sciences Education, vol. 12, no. 2, pp. 206-214, 2013.

[3] R. J. Dufresne, W. J. Gerace, and W. J. Leonard, "Solving physics problems with multiple representations," The Physics Teacher, vol. 35, no. 5, p. 270, 1997.

[4] M. M. Cooper, N. Grove, S. M. Underwood, and M. W. Klymkowsky, "Lost in lewis structures: An investigation of student difficulties in developing representational competence," Journal of Chemical Education, vol. 87, no. 8, pp. 869$874,2010$.

[5] S. Ainsworth, "The functions of multiple representations," Computers $\mathcal{E} E d u$ cation, vol. 33, pp. 131-152, 1999.

[6] M. T. H. Chi, P. J. Feltovich, and R. Glaser, "Categorization and representations of physics problems by experts and novices," Cognitive Science, vol. 5, pp. 121-152, 1981.

[7] D. Rosengrant, E. Etkina, and A. V. Heuvelen, "An Overview of Recent Research on Multiple Representations," AIP Conference Proceedings, vol. 883, pp. 149-153, 2007.

[8] B. E. Hinrichs, "Using the system schema representational tool to promote student understanding of Newton's Third Law," Physics Education Research Conference Proceedings, vol. 1, pp. 117-120, 2004.

[9] S. Ainsworth, D. Wood, and C. O. Malley, "There is more than one way to solve a problem: evaluating a learning environment that supports the development of children's multiplication skills," Learning and Instruction, vol. 8, no. 2, pp. 141157, 1998.

[10] D. L. Schwartz, "The emergence of abstract representations in dyad problem solving," The Journal of the Learning Sciences, vol. 4, no. 3, pp. 321-354, 1995.

[11] T. Fredlund, C. Linder, J. Airey, and A. Linder, "Unpacking physics representations : Towards an appreciation of disciplinary affordance," Physical Review Special Topics - Physics Education Research, vol. 10, p. 020129, 2014. 
[12] R. Cox and P. Brna, "Supporting the Use of External Representations in Problem Solving : the Need for Flexible Learning Environments," Journal of Artificial Intelligence in Education, vol. 6, pp. 239-302, 1995.

[13] E. Brewe, "Energy as a substancelike quantity that flows: Theoretical considerations and pedagogical consequences," Physical Review Special Topics - Physics Education Research, vol. 7, no. 2, 2011.

[14] D. McPadden, J. Dowd, and E. Brewe, "Power Boxes: New representation for analyzing DC circuits," The Physics Teacher (accepted).

[15] M. M. Cooper, S. M. Underwood, C. Z. Hilley, and M. W. Klymkowsky, "Development and assessment of a molecular structure and properties learning progression," Journal of Chemical Education, vol. 89, no. 11, pp. 1351-1357, 2012.

[16] M. Yerushalmy, "Student perceptions of aspects of algebraic function using multiple representation software," Journal of Computer Assisted Learning, vol. 7, pp. 42-57, 1991.

[17] Y. Uesaka, E. Manalo, and S. Ichikawa, "What kinds of perceptions and daily learning behaviors promote students' use of diagrams in mathematics problem solving?," Learning and Instruction, vol. 17, pp. 322-335, 2007.

[18] A. Andrea and B. L. Sherin, "Meta-representation : an introduction," Mathematical Behavior, vol. 19, pp. 385-398, 2000.

[19] P. B. Kohl and N. D. Finkelstein, "Patterns of multipe representation use by experts and novices during physics problem solving," Physical Review Special Topics - Physics Education Research, vol. 4, no. 1, pp. 1-13, 2008.

[20] A. Maries, S.-y. Lin, and C. Singh, "Challenges in designing appropriate scaffolding to improve students ' representational consistency : The case of a Gauss ' s law problem," Physical Review Physics Education Research, vol. 13, p. 020103, 2017.

[21] A. F. Heckler, "Some Consequences of Prompting Novice Physics Students to Construct Force Diagrams," International Journal of Science Education, vol. 32, no. 14, pp. 1829-1851, 2010.

[22] E. Kuo, N. R. Hallinen, and L. D. Conlin, "When procedures discourage insight: epistemological consequences of prompting novice physics students to construct force diagrams," International Journal of Science Education, vol. 0693, no. April, pp. 1-26, 2017.

[23] M. D. Cock, "Representation use and strategy choice in physics problem solving," Physical Review Special Topics - Physics Education Research, vol. 8, p. $020117,2012$. 
[24] D. E. Meltzer, "Relation between students' problem-solving performance and representational format," American Journal of Physics, vol. 73, no. 5, pp. 463478, 2005.

[25] P. B. Kohl and N. D. Finkelstein, "Student representational competence and self-assessment when solving physics problems," Physical Review Special Topics - Physics Education Research, vol. 1, p. 010104, 2005.

[26] E. Brewe, "Modeling theory applied: Modeling Instruction in introductory physics," American Journal of Physics, vol. 76, no. 12, p. 1155, 2008.

[27] E. Brewe and V. Sawtelle, "Modeling Instruction for University Physics: Examining the Theory in Practice," European Journal of Physics (submitted in this issue).

[28] D. Hestenes, "Toward a modeling theory of physics instruction," American Journal of Physics, vol. 55, no. 5, pp. 440-454, 1987.

[29] N. J. Nersessian, "Maxwell and "the Method of Physical Analogy": Modelbased Reasoning, Generic Abstraction, and Conceptual Change," in Reading Natural Philosophy: Essays in the History and Philosophy of Science and Mathematics (D. B. Malament, ed.), ch. 6, pp. 129-166, Peru: Open Court Publishing Company, 2002.

[30] NGSS Lead States, Next Generation Science Standards: For States, By States. Washington, DC: The National Academies Press, 2013.

[31] J. Kozminski, N. Beverly, D. Deardorff, R. Dietz, M. Eblen-Zayas, R. Hobbs, H. Lewandowski, S. Lindaas, A. Reagan, R. Tagg, J. Williams, and B. Zwickl, "AAPT Recommendations for the Undergraduate Physics Laboratory Curriculum," American Association of Physics Teachers, p. 29, 2014.

[32] I. A. Halloun, Modeling Theory in Science Education. 1st ed., 2006.

[33] B. Rogoff, "Explanations of Cognitive Development through Social Interaction: Vygotsky and Piaget," in Apprenticeship in Thinking: Cognitive Development in Social Context, pp. 1-16, 1990.

[34] D. M. Desbien, Modeling discourse management compared to other classroom management styles in university physics. Doctoral dissertation, Arizona State University, 2002.

[35] J. Durden, E. Brewe, and L. Kramer, "'Implicit action": Understanding discourse management in modeling instruction," AIP Conference Proceedings, vol. 1413, pp. 187-190, 2012.

[36] M. Wells, D. Hestenes, and G. Swackhamer, "A Modeling Method for high school physics instruction," Am J Phys, vol. 63, no. 7, pp. 606-619, 1995. 
[37] "University Modeling Instruction - https://univ-modelinginstruction.com."

[38] E. Brewe, V. Sawtelle, L. H. Kramer, G. E. O'Brien, I. Rodriguez, and P. Pamelá, "Toward equity through participation in Modeling Instruction in introductory university physics," Physical Review Special Topics - Physics Education Research, vol. 6, no. 1, pp. 1-12, 2010.

[39] D. Hestenes, M. Wells, and G. Swackhamer, "Force concept inventory," The Physics Teacher, vol. 30, no. 3, pp. 141-158, 1992.

[40] R. K. Thornton and D. R. Sokoloff, "Assessing student learning of Newton's laws: The Force and Motion Conceptual Evaluation and the Evaluation of Active Learning Laboratory and Lecture Curricula," American Journal of Physics, vol. 66, no. 4, pp. 338-352, 1998.

[41] E. Brewe, A. Traxler, J. De La Garza, and L. H. Kramer, "Extending positive CLASS results across multiple instructors and multiple classes of Modeling Instruction," Physical Review Special Topics - Physics Education Research, vol. 9, no. 2, pp. 1-10, 2013.

[42] W. K. Adams, K. K. Perkins, N. S. Podolefsky, M. Dubson, N. D. Finkelstein, and C. E. Wieman, "New instrument for measuring student beliefs about physics and learning physics: The Colorado Learning Attitudes about Science Survey," Physical Review Special Topics - Physics Education Research, vol. 2, no. 1, pp. 1-14, 2006.

[43] A. Madsen, S. B. McKagan, and E. C. Sayre, "How physics instruction impacts students' beliefs about learning physics: A meta-analysis of 24 studies," Physical Review Special Topics - Physics Education Research, vol. 11, no. 1, pp. 1-19, 2015 .

[44] R. M. Goertzen, E. Brewe, and L. Kramer, "Expanded Markers of Success in Introductory University Physics," International Journal of Science Education, vol. 35, no. 2, pp. 262-288, 2013.

[45] R. W. Chabay and B. A. Sherwood, Matter and Interactions. Hoboken: John Wiley \& Sons, Inc., 3rd ed., 2011.

[46] H. D. Young, R. A. Freedman, A. L. Ford, and F. W. Sears, University Physics: with Modern Physics. San Francisco: Pearson Addison Wesley, 14th ed., 2004.

[47] J. Kaput, "Linking representations in the symbol systems of algebra," in Research issues in the learning and teaching of algebra (S. Wagner and C. Kieran, eds.), pp. 167-194, New York: Macmillan, 1989.

[48] H. J. M. Tabachneck, K. R. Koedinger, and M. J. Nathan, "Towards a theoretical account of strategy use and sense making in mathematical problem 
solving," in Proceedings of the 16th annual conference of the cognitive science society (A. Ram and K. Eiselt, eds.), pp. 836-847, Hillsdale: Erlbaum, 1994.

[49] C. J. De Leone and E. Gire, "Is instructional emphasis on the use of nonmathematical representations worth the effort?," Physics Education Research Conference Proceedings, vol. 818, pp. 45-48, 2005.

[50] D. Hestenes, "Modeling Methodology for Physics Teachers," Proceedings of the International Conference on Undergraduate Physics Education (AIP), vol. 399, no. 1, pp. 935-958, 1996.

[51] D. Rosengrant, A. Van Heuvelen, and E. Etkina, "Free-body diagrams: Necessary or sufficient?," Physics Education Research Conference Proceedings, vol. 1, pp. 177-180, 2004.

[52] J. I. Heller and F. Reif, "Prescribing Effective Human Problem-Solving Processes: Problem Description in Physics," Cognition and Instruction, vol. 1, no. 2, pp. 177-216, 1984.

[53] A. Van Heuvelen and X. Zou, "Multiple representations of workenergy processes," American Journal of Physics, vol. 69, no. 2001, p. 184, 2001.

[54] M. Van Sommeren, P. Reimann, H. Boshuizen, and T. de Jong, Learning with Multiple Representations. Amsterdam: Pergamon, 1998.

[55] R. J. Beichner, "Testing student interpretation of kinematics graphs," American Journal of Physics, vol. 62, no. 8, pp. 750-762, 1994.

[56] L. C. McDermott, M. L. Rosenquist, and E. H. van Zee, "Student difficulties in connecting graphs and physics: Examples from kinematics," American Journal of Physics, vol. 55, no. 6, pp. 503-513, 1987.

[57] S. N. Friel, F. R. Curcio, and G. W. Bright, "Making sense of graphs: Critical factors influencing comprehension and instructional implications," Journal for Research in Mathematics Education, vol. 32, no. 2, pp. 124-158, 2001.

[58] D.-h. Nguyen and N. S. Rebello, "Students ' understanding and application of the area under the curve concept in physics problems," Physical Review Special Topics - Physics Education Research, vol. 7, p. 010112, 2011.

[59] C. Trujillo, M. M. Cooper, and M. W. Klymkowsky, "Using graph-based assessments within socratic tutorials to reveal and refine students' analytical thinking about molecular networks," Biochemistry and Molecular Biology Education, vol. 40, no. 2, pp. 100-107, 2012.

[60] B. L. Sherin, "How students understand physics equations," Cognition and Instruction, vol. 19, no. 4, pp. 479-541, 2001. 
[61] T. J. Bing and E. F. Redish, "Analyzing problem solving using math in physics : Epistemological framing via warrants," Physical Review Special Topics - Physics Education Research, vol. 5, p. 020108, 2009.

[62] D.-h. Nguyen and N. S. Rebello, "Students' difficulties with integration in electricity," Physical Review Special Topics - Physics Education Research, vol. 7, p. 010113, 2011.

[63] J. H. Larkin and H. A. Simon, "Why a Diagmm is (Sometimes) Worth Ten Thousand Words," Cognitive Science, vol. 11, pp. 65-99, 1987.

[64] R. E. Scherr, H. G. Close, E. W. Close, and S. Vokos, "Representing energy. II. Energy tracking representations," Physical Review Special Topics - Physics Education Research, vol. 8, no. 2, 2012.

[65] R. E. Scherr, H. G. Close, S. B. McKagan, and S. Vokos, "Representing energy. I. Representing a substance ontology for energy," Physical Review Special Topics - Physics Education Research, vol. 8, no. 2, pp. 1-11, 2012.

[66] N. D. Finkelstein, W. K. Adams, C. J. Keller, P. B. Kohl, K. K. Perkins, N. S. Podolefsky, S. Reid, and R. Lemaster, "When learning about the real world is better done virtually: A study of substituting computer simulations for laboratory equipment," Physical Review Special Topics - Physics Education Research, vol. 1, no. 1, pp. 1-8, 2005.

[67] A. L. Traxler, J. V. Mahadeo, D. McPadden, and E. Brewe, "Multiple Representations and Epistemic Games in Introductory Physics Exam Solutions," 2014 Physics Education Research Conference Proceedings, pp. 247-250, 2014.

[68] A. Bakker and J. Derry, "Lessons from Inferentialism for Statistics Education," Mathematical Thinking and Learning, vol. 13, no. 1-2, pp. 5-26, 2011.

[69] V. Sawtelle, E. Brewe, and L. H. Kramer, "Positive impacts of modeling instruction on self-efficacy," AIP Conference Proceedings, vol. 1289, pp. 289-292, 2010 .

[70] I. Rodriguez, G. Potvin, E. Brewe, and L. H. Kramer, "The Impacts of Modeling Physics in Upper-Level Courses: The Persistence of Males and Females," 2014 Physics Education Research Conference Proceedings, pp. 219-222, 2014.

[71] K. A. Douglas, A. Rynearson, S. Purzer, and J. Strobel, "Reliability, Validity , and Fairness : A Content Analysis of Assessment Development Publications in Major Engineering Education Journals *," International Journal of Engineering Education, vol. 32, no. 5, pp. 1960-1971, 2016.

[72] A. L. Traxler, X. C. Cid, J. Blue, and R. Barthelemy, "Enriching gender in PER: A binary past and a complex future," arXiv preprint arXiv:150\%.0510\%, vol. 12, pp. 020114:1-15, 2016. 
[73] F. Wilcoxon, "Individual Comparisons by Ranking Methods," Biometrics Bulletin, vol. 1, no. 6, pp. 80-83, 1945.

[74] H. B. Mann and D. R. Whitney, "Institute of Mathematical Statistics is collaborating with JSTOR to digitize, preserve, and extend access to The Annals of Mathematical Statistics. ( $)$ www.jstor.org," The Annals of Mathematical Statistics, vol. 18, no. 1, pp. 50-60, 1947.

[75] M. P. Fay and M. a. Proschan, "Wilcoxon-Mann-Whitney or t-test? On assumptions for hypothesis tests and multiple interpretations of decision rules," Statistics Surveys, vol. 4, no. 0, pp. 1-39, 2010.

[76] A. Y. Gordon, G. Glazko, X. Qiu, and A. Yakovlev, "Control of the mean number of false discoveries, Bonferroni and stability of multiple testing," The Annals of Applied Statistics, vol. 1, no. 1, pp. 179-190, 2007.

[77] A. V. Frane, "Are Per-Family Type I Error Rates Relevant in Social and Behavioral Science?," Journal of Modern Applied Statistical Methods, vol. 14, no. 1, pp. 12-23, 2015.

[78] N. Cliff, "Dominance statistics: Ordinal analyses to answer ordinal questions.," Psychological Bulletin, vol. 114, no. 3, pp. 494-509, 1993.

[79] J. Cohen, Statistical Power Analysis for the Behavioral Sciences. New York: Academic Press, 1969.

[80] J. Romano, J. D. Kromrey, J. Coraggio, J. Skowronek, and L. Devine, "Exploring methods for evaluating group differences on the NSSE and other surveys: Are the t-test and Cohen's d indices the most appropriate choices?," Annual meeting of the Southern Association for Institutional Research, pp. 14-17, 2006.

[81] D. McPadden and E. Brewe, "Network Analysis of Students' Representation Use in Problem Solving," 2014 Physics Education Research Conference Proceedings, pp. 219-222, 2015.

[82] P. S. Shaffer and L. C. McDermott, "Research as a guide for curriculum development: An example from introductory electricity. Part II: Design of instructional strategies," American Journal of Physics, vol. 60, no. 11, pp. 1003-1013, 1992.

[83] D. R. Dounas-Frazer, K. L. Van De Bogart, M. R. Stetzer, and H. J. Lewandowski, "Investigating the role of model-based reasoning while troubleshooting an electric circuit," Physical Review Physics Education Research, vol. 12, no. 1, pp. 1-20, 2016.

[84] B. M. Zwickl, D. Hu, N. Finkelstein, and H. J. Lewandowski, "Model-based reasoning in the physics laboratory: Framework and initial results," Physical Review Special Topics - Physics Education Research, vol. 11, no. 2, pp. 1-12, 2015. 
[85] M. Black, Models and metaphors: Studies in language and philosophy. Ithaca, NY: Cornell University Press, 1962.

[86] E. Etkina and A. Van Heuvelen, "Investigative Science Learning Environment: Using the processes of science and cognitive strategies to learn physics," Proceedings of the 2001 Physics Education Research Conference, pp. 17-20, 2001.

[87] P. W. Irving, M. J. Obsniuk, and M. D. Caballero, "P3: A practice focused learning environment," European Journal of Physics, vol. 38, no. 5, pp. 1-13, 2017.

[88] M. Windschitl, J. Thompson, and M. Braaten, "Beyond the scientific method: Model-based inquiry as a new paradigm of preference for school science investigations," Science Education, vol. 92, no. 5, pp. 941-967, 2008.

[89] E. Brewe, L. Kramer, and V. Sawtelle, "Investigating student communities with network analysis of interactions in a Physics Learning Center," Physical Review Special Topics - Physics Education Research, vol. 8, p. 010101, 2012.

[90] I. Rodriguez, G. Potvin, and L. H. Kramer, "How gender and reformed introductory physics impacts student success in advanced physics courses and continuation in the physics major," Physical Review Physics Education Research, no. 12, p. 020118, 2016.

[91] S. Freeman, S. L. Eddy, M. McDonough, M. K. Smith, N. Okoroafor, H. Jordt, and M. P. Wenderoth, "Active learning increases student performance in science, engineering, and mathematics," Proceedings of the National Academy of Sciences, vol. 111, no. 23, pp. 8410-8415, 2014.

[92] B. Forsthuber, A. Motiejunaite, and A. S. de Almeida Coutinho, Science Education in Europe: National Policies, Practices, and Research. Education, Audiovisual and Culture Executive Agency, European Commission, 2011.

[93] V. L. Akerson, J. S. Townsend, L. A. Donnelly, D. L. Hanson, P. Tira, and O. White, "Scientific Modeling for Inquiring Teachers Network (SMIT'N): The influence on elementary teachers' views of nature of science, inquiry, and modeling," Journal of Science Teacher Education, vol. 20, no. 1, pp. 21-40, 2009.

[94] L. C. McDermott and P. S. Shaffer, Tutorials in Introductory Physics. Prentice Hall, 1998.

[95] University of Maryland-PER Group, "Activity Based Physics Tutorials: https://www.physics.umd.edu/perg/abp/abptutorials/."

[96] D. McPadden and E. Brewe, "Impact of the second semester University Modeling Instruction course on students' representation choices," Physical Review Physics Education Research, vol. 13, no. 2, p. 020129, 2017. 
[97] R. Driver and L. Warrington, "Students' Use of the Principle of Energy Conservation in Problem Situations," Physics Education, vol. 20, no. 4, pp. 171-176, 1985.

[98] "About FIU - https://www.fiu.edu/about/index.html."

[99] D. P. Maloney, T. L. O’Kuma, C. J. Hieggelke, and A. Van Heuvelen, "Surveying students' conceptual knowledge of electricity and magnetism," American Journal of Physics, vol. 67, no. 7, pp. S12-S23, 2001.

[100] V. P. Engelhardt and R. J. Beichner, "Students' understanding of direct current resistive electrical circuits," American Journal of Physics, vol. 72, no. 1, pp. 98$115,2004$.

[101] J. Bransford and D. Schwartz, "Rethinking Transfer : A Simple Proposal with Multiple Implications Author ( s ): John D . Bransford and Daniel L . Schwartz Source : Review of Research in Education , Vol . 24 , ( 1999 ), pp . 61-100 Published by : American Educational Research Associatio," Review of Research in Education, vol. 24, no. 1999, pp. 61-100, 1999.

[102] K. Duncker and L. S. Lees, "On problem-solving.," Psychological Monographs, vol. 58, no. 5, pp. i-113, 1945.

[103] M. L. Gick and K. J. Holyoak, "Analogical problem solving," Cognitive Psychology, vol. 12, no. 3, pp. 306-355, 1980.

[104] S. K. Reed, G. W. Ernst, and R. Banerji, "The role of analogy in transfer between similar problem states," Cognitive Psychology, vol. 6, no. 3, pp. 436450, 1974.

[105] J. R. Anderson, L. M. Reder, and H. A. Simon, "Situated Learning and Education Situated Learning and Education'," Educational Researcher, vol. 25, no. 4, pp. 5-11, 1996.

[106] N. S. Rebello, L. Cui, A. G. Bennett, D. A. Zollman, and D. J. Ozimek, "Transfer of learning in problem solving in the context of mathematics and physics," in Learning to solve complex scientific problems, pp. 223-246, 2007.

[107] J. W. Clark, J. R. Thompson, and D. B. Mountcastle, "Investigating Student Conceptual Difficulties in Thermodynamics Across Multiple Disciplines: The First Law and P-V Diagrams," ASEE Conference Proceedings, 2014.

[108] D. McPadden, J. E. Dowd, and E. Brewe, "Extending University Modeling Instruction to Introductory Electricity and Magnetism," European Journal of Physics (submitted). 
[109] K. A. Douglas and S. Purzer, "Validity : Meaning and Relevancy in Assessment for Engineering Education Research," Journal of Engineering Education, vol. 104, no. 2, pp. 108-118, 2015.

[110] M. G. Bell and Y. Iida, Transportation Network Analysis. 1997.

[111] Y.-Y. Ahn, S. E. Ahnert, J. P. Bagrow, and A.-L. Barabási, "Flavor network and the principles of food pairing," pp. 1-7, 2011.

[112] D. Z. Grunspan, B. L. Wiggins, and S. M. Goodreau, "Understanding classrooms through social network analysis: A primer for social network analysis in education research," CBE Life Sciences Education, vol. 13, no. 2, pp. 167-178, 2014 .

[113] M. E. J. Newman, "The structure of scientific collaboration networks," Proceedings of the National Academy of Sciences of the United States of America, vol. 98, no. 2, pp. 404-409, 2001.

[114] J. Bruun, Networks in Physics Education. Doctoral dissertation, University of Copenhagen, 2012.

[115] E. Brewe, J. Bruun, and I. G. Bearden, "Using module analysis for multiple choice responses: A new method applied to Force Concept Inventory data," Physical Review Physics Education Research, vol. 12, no. 2, pp. 020131:1-19, 2016.

[116] R. Dou, E. Brewe, J. P. Zwolak, G. Potvin, E. A. Williams, and L. H. Kramer, "Beyond performance metrics: Examining a decrease in students' physics selfefficacy through a social networks lens," Physical Review Physics Education Research, vol. 12, no. 2, pp. 1-14, 2016.

[117] N. J. Foti, J. M. Hughes, and D. N. Rockmore, "Nonparametric sparsification of complex multiscale networks," PLOS ONE, vol. 6, no. 2, p. e16431, 2011.

[118] M. A. Serrano, M. Boguna, and A. Vespignani, "Extracting the multiscale backbone of complex weighted networks," Proceedings of the National Academy of Sciences, USA, vol. 106, no. 16, pp. 6483-6488, 2009.

[119] J. P. Bagrow, "Personal Correspondence," 2018.

[120] J. Nieminen, "On the centrality in a graph," Scandinavian Journal of Psychology, vol. 15, pp. 332-336, 1974.

[121] P. Bonacich, "Power and Centrality: A Family of Measures," American Journal of Sociology, vol. 92, no. 5, pp. 1170-1182, 1987.

[122] L. C. Freeman, "A Set of Measures of Centrality Based on Betweenness," Sociometry, vol. 40, no. 1, pp. 35-41, 1977. 
[123] S. Adams, "The 10 Skills Employers Most Want In 2015 Graduates," 2014. 


\section{APPENDICES}

\section{Problem Solving and Representation Use Survey (PSRUS)}

The following addresses the development of the Problem Solving and Representation Use Survey (PSRUS), which was used in the analyzes for Chapters 2-4. The current appendix describes the purpose of the survey, validation measures taken, and limitations of the survey. A copy of the PSRUS given in the MI-EM classroom follows. The final page of this appendix shows the list of representations taken from the MI curricula, which was also distributed to students with the PSRUS.

\section{Purpose of the Survey}

The PSRUS was designed to examine the representation choices that students make across the introductory physics content covered in the MI courses. Designed after a modified card-sort survey, we asked students to list the representations they would use to solve each physics question or to write in the representation they would use if they did not see it as listed. The questions were designed to cover the content from introductory mechanics and electricity and magnetism (EM), with many parallel questions between the two contexts. In its design, the PSRUS is meant to examine the representations that students see as useful and what representations they would see as useful together in solving problems from both mechanics and EM. This survey was not intended to study whether students were choosing the "correct" representations or the most efficient representations. Thus, we did not attempt to score this survey, compare student responses to faculty responses, or in any way relate student responses to established solution paths, which affects both the validation measures that we take and the interpretation of our results. 


\section{Validation, Reliability, and Fairness}

In the development of this survey, we addressed several aspects of validity including: content/face validity, substantive validity, construct validity (internal consistency), fairness, and multiple-form reliability. To address content/face validity and substantive validity, we gave the survey to a test panel consisting of three faculty members, one postdoctoral researcher, six graduate students, and two undergraduate students. As described in Chapter 2, we asked the faculty and graduate students to judge the appropriateness of the questions for introductory physics students and whether the questions were appropriate for measuring a broad range of students' representation use in order to address content and face validity in our survey [71]. Fairness was addressed by ensuring the test panel was diverse, including representation from: multiple genders, multiple ethnic and racial groups, and bilingual/non-native English speakers. Since the survey was given at a large, Hispanic-serving institution, it was particularly important that the survey be fair and understandable by members of these populations [71]. Multiple-form reliability was addressed by creating two forms of the survey - one of which ordered the EM questions first and one which ordered the mechanics questions first. We found no difference in the number or variety of representations chosen on each form of the survey.

Substantive validity, which asks if the intended audience is able to read, respond and engage with the instrument in the intended manner [71], was addressed by asking the undergraduate students on the test panel to comment on the wording of the questions and instructions as well as the amount of time required for the survey. Additionally, our panel consisted of people who had a wide range of exposure to Modeling Instruction: there were developers of Modeling Instruction, students and 
instructors who had previously taken or taught Modeling Instruction courses, and students and instructors with no exposure to the Modeling Instruction courses. So this suggests that the PSRUS is interpretable and substantively valid [71] for people who have been in Modeling Instruction before and those who have not.

To further judge the substantive validity (whether the intended audience is able to engage with the instrument in the intended manner) and the construct validity, we examined student responses to each question, particularly looking at the total number of representations chosen and the percent breakdown of which representations students chose for each given question. The responses from the Spring 2015 prePSRUS shown in Figures 20 - 22. (The responses from the Spring 2015 post-PSRUS, Spring 2016 pre-PSRUS, and Spring 2016 post-PSRUS were similar and are shown in the subsequent appendices.) From these pie charts, we see that there is a varying number of representations chosen in total for each question and that the proportion of each representation varies by question. This shows that students are not simply listing every representation as relevant for every question. For example, there is a higher proportion of equations chosen on Question 3, which asks for a car's acceleration, than on Question 2, which asks for an asteroid's path (as shown in Figure 20). This further demonstrates that students are engaging with the instrument as intended and are providing responses that depend contextually on each question.

Because the PSRUS was not intended to be graded or scored, we did not address validation measures such as the item discrimination or item difficulty, which are typically included as a part of content validity and structural validity [71]. We also did not use the coefficient alpha (Cronbach's alpha) or Kuder-Richardson Formula as an evaluation of the PSRUS's internal consistency as each item is neither scored 

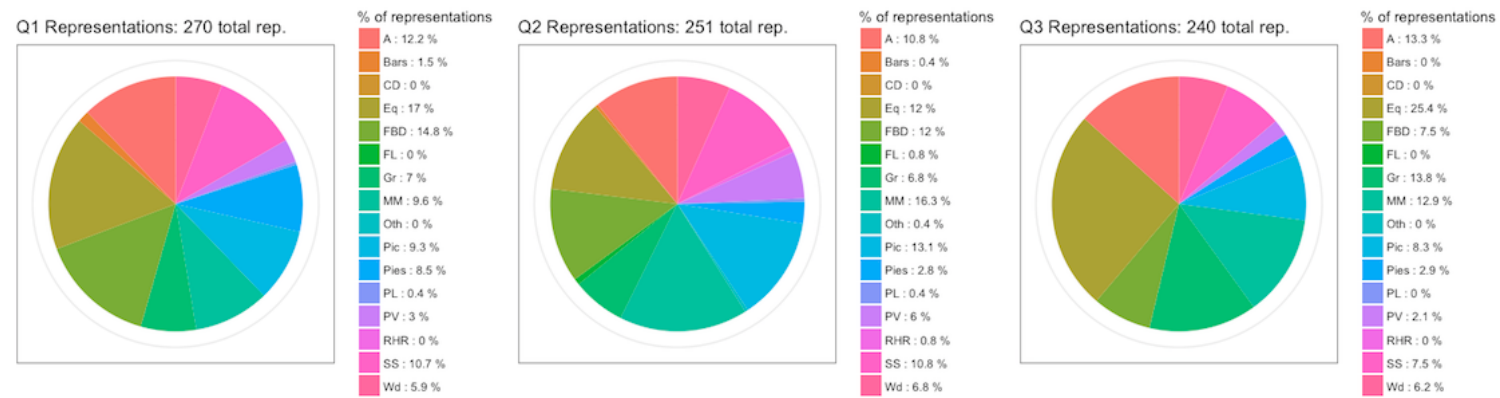

Figure 20: This figure shows the total number of representations chosen for Questions 1-3 on the Spring 2015 pre-PSRUS, including the breakdown (by percent) of which representations were chosen.

nor made up of dichotomous choices. These additional measures of validity would need to be assessed if the PSRUS were to be used to judge the efficiency of students' representation choices (or some alternate scoring method).

\section{Limitations and Interpretation of Results}

The first and foremost limitation of the PSRUS is that we only asked students to list the representations that they would use on these problems. This means that we are measuring the number of representations that students think they will need, not what they actually use to solve each question. From the standpoint taken in this dissertation of representations as tools in problem solving, we argue that studying the representations students think will be useful is informative in itself as it highlights the tools that students would draw on in their problem solving.

From this same viewpoint of representations as tools, increasing the number of representations that students see as useful and coordinated serves to increase the likelihood of success in problem solving [7, 49] and facilitates deeper conceptual understanding [5]. The MI courses (both MI-Mech and MI-EM) are, in part, designed 

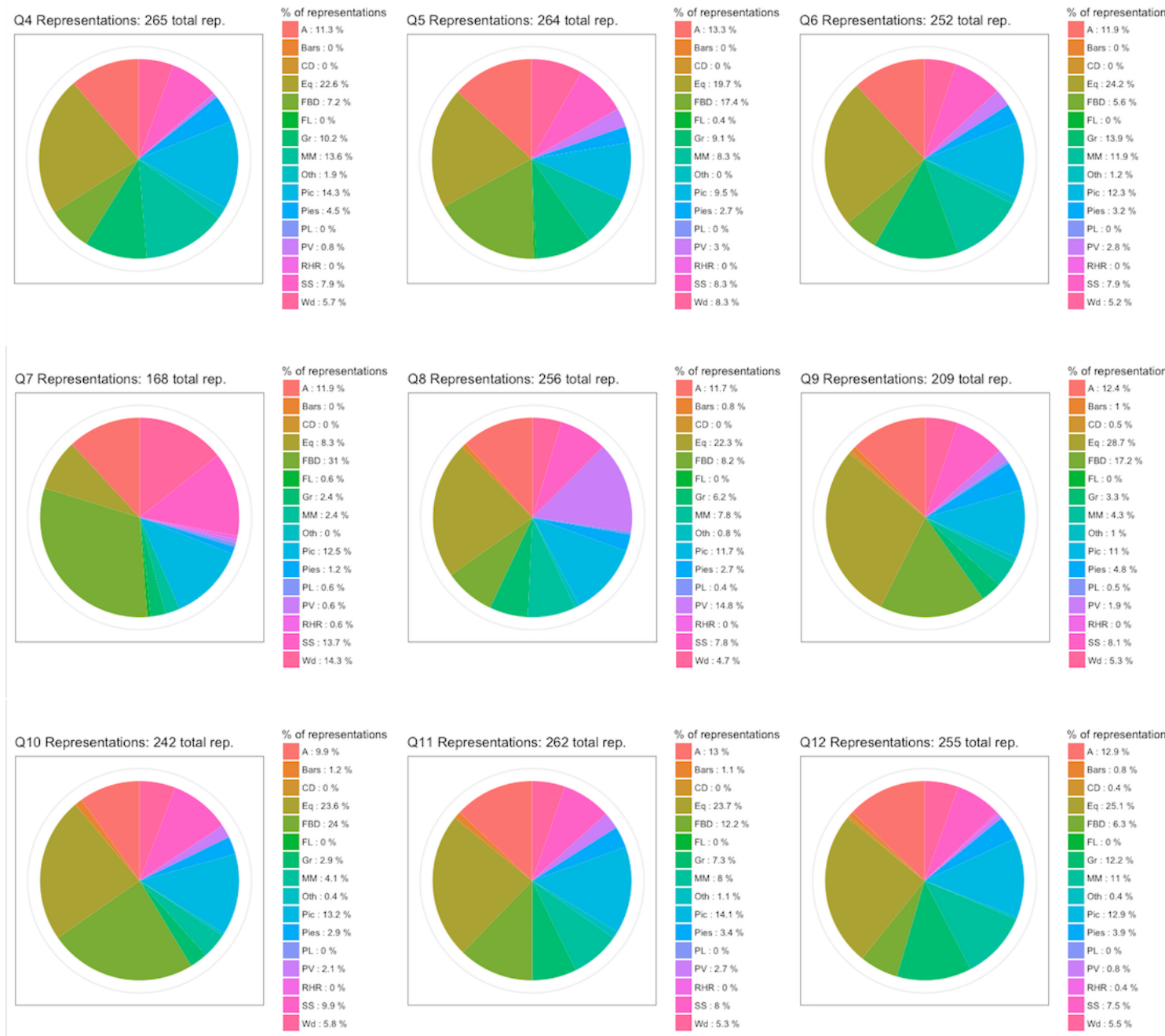

Q13 Representations: 162 total rep.
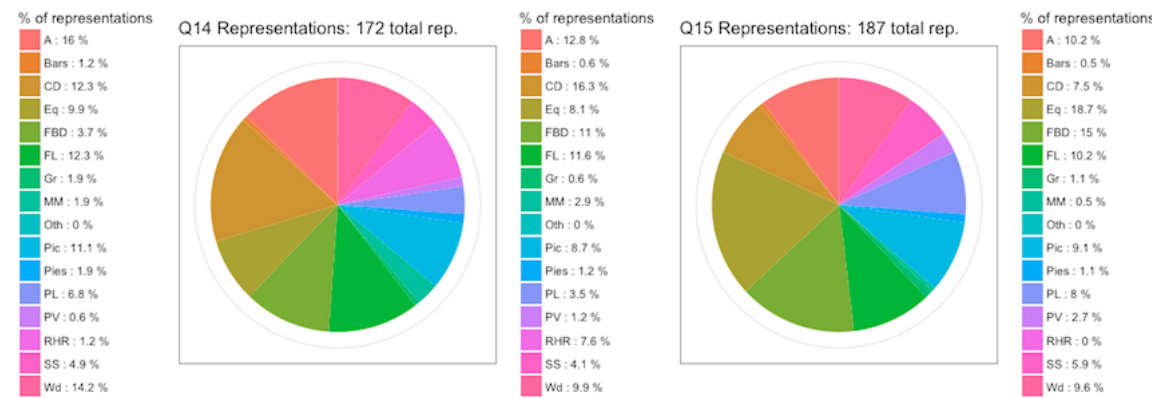

Figure 21: This figure shows the total number of representations chosen for Questions 4-15 on the Spring 2015 pre-PSRUS, including the breakdown (by percent) of which representations were chosen. 

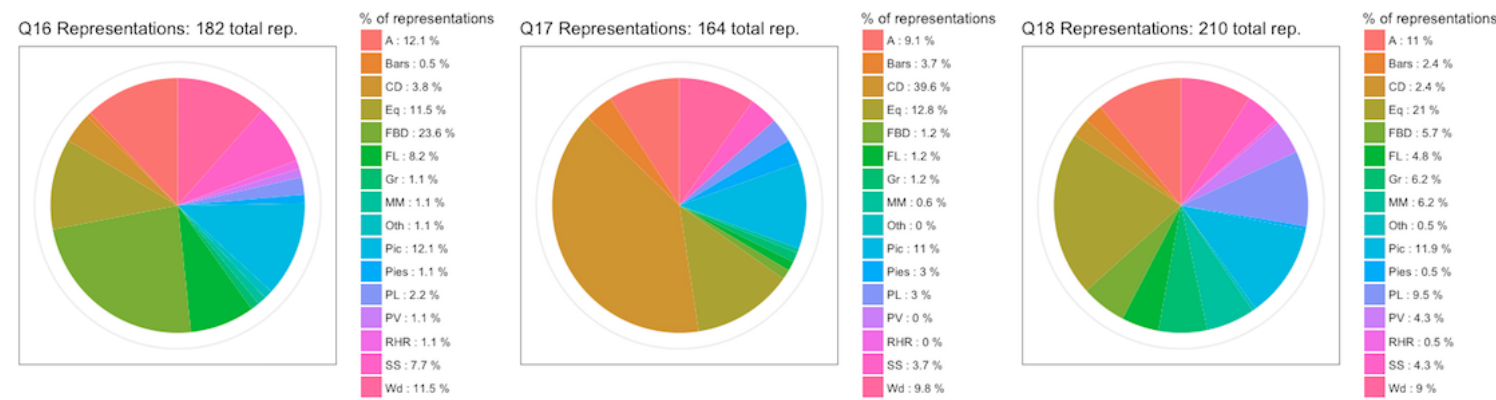

Q19 Representations: 180 total rep.
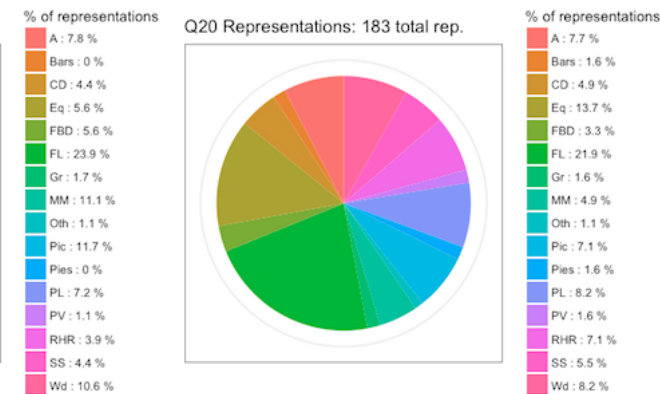

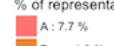

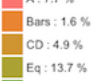

ED: $4.9 \%$

FBD : $3.3 \%$
FL: $21.9 \%$
Gr: $1.6 \%$

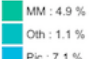

Pic : $1.1 \%$

Pies $: 1.6 \%$
$P L: 8.2 \%$

PV: $1.6 \%$

SS : $5.5 \%$

Wd: $8.2 \%$

Q21 Representations: 187 total rep. $\%$ of represen

$\%$ of representations

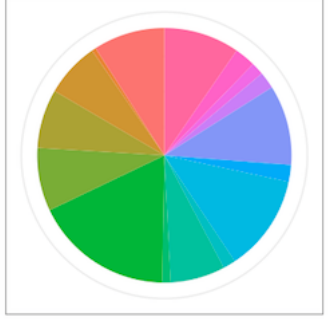

Bars: $0.5 \%$

Eq: $7.5 \%$

FBD: $8 \%$

$\mathrm{FL}: 17.6 \%$
$\mathrm{Gr}: 1.1 \%$

Gr: $1.1 \%$

Eth: $1.6 \%$

Pic: $12.3 \%$

Pies: $21 \%$

PV: $2.1 \%$

RHR: $1.6 \%$
SS : $2.7 \%$

Wo: $: 9.6 \%$

Q22 Representations: 161 total rep.
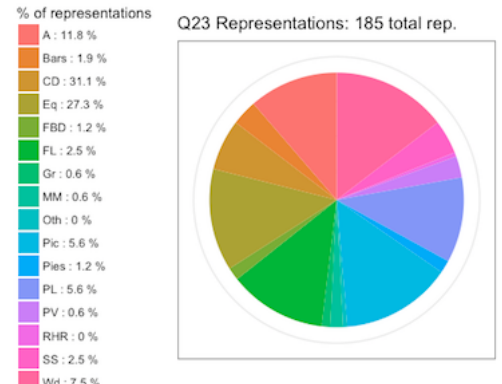

$\%$ of representations

A: $11.4 \%$

Bars: $3.2 \%$

Eq: $13 \%$

FBD $1.6 \%$

$\mathrm{FL}: 12.4 \%$
$\mathrm{Gr}: 1.1 \%$
$\mathrm{D}_{\mathrm{MM}: 1.6 \%}$

Oth: $0.5 \%$

Pic : $14.1 \%$
Pies : $1.6 \%$
$P L: 10.8 \%$

PL: $10.8 \%$

RHR: $0.5 \%$

Ss: $: 3 \%$
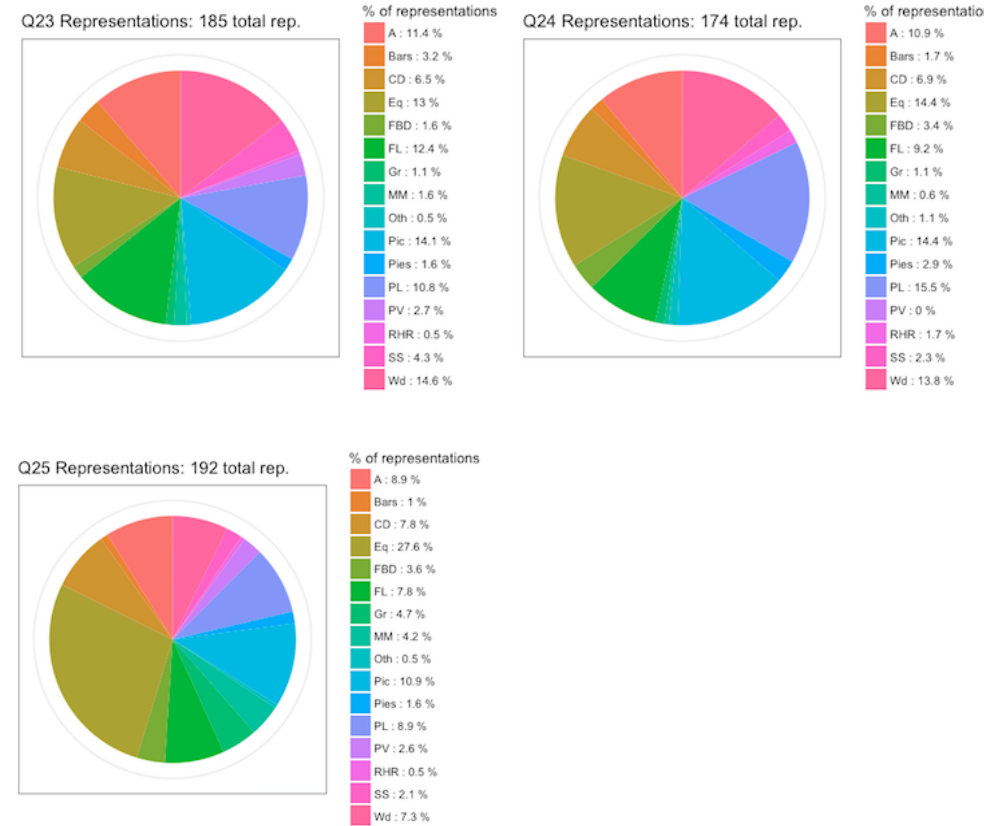

Figure 22: This figure shows the total number of representations chosen for Questions 6-25 on the Spring 2015 pre-PSRUS, including the breakdown (by percent) of which representations were chosen. 
with representation use and coordination as an explicit learning goal. Use of multiple representations features heavily in the curriculum materials, in student assessment (on homework, formal labs, and exams), and in the day-to-day activities of the class. Thus, we argue that a positive outcome particularly for this class is an increase in representations over the course of the semester. As a result, the work in this dissertation has focused on the differences in the number, variety, and co-occurance of the representation choices that MI-EM students make over the course of the semester.

We recognize that an increase in number or variety of representations is not a positive outcome in all contexts. In fact, as expertise is developed, there may be a decrease in the number of representations chosen as experts frequently skip steps or no longer require the external representations as a part of their problem solving process. Instead, Kohl and Finkelstein have shown that rather than using more representations, experts are able to translate between representations more easily [19]. If the PSRUS were to be given in upper-division courses, the interpretation of changes in representation choices would be less clear. Thus, we have limited the scope of the PSRUS (and its interpretation) to the introductory physics classrooms, where such expertise is likely still undeveloped.

Finally, the PSRUS was developed to particularly examine students' representation choices in MI-EM. As a result, there were several representations included on the given sheet with the PSRUS that were developed for the MI courses that we would not expect new students to be familiar with, namely system schema, energy pie charts, and energy bar charts. Therefore, we would expect new students to have a lower average number of representations per question (as shown in Figures 4 and 14) due to their unfamiliarity with several of the listed representations. 


\section{Problem Solving and Representation Use Survey}

Directions: For each situation listed below, please indicate which of the tool(s)/ representation(s) on the last page you would use to solve the problem. We are interested in what you would use to solve the problems - there are no wrong or right answers here.

Note: Please do NOT actually solve each problem! Just indicate which tool(s)/ representation(s) you would use if you were going to solve the problem.

1. A phone slides across the table and comes to a stop. How much work was done by the table on the phone?

Representation(s):

2. An asteroid falls toward a planet. What path does the asteroid take?

Representation(s):

3. A car that is initially traveling at $45 \mathrm{mph}$ comes to rest at a stoplight in 15 seconds. What is the car's acceleration while stopping?

Representation(s):

4. A frisbee is thrown straight up in the air with an initial speed $3 \mathrm{~m} / \mathrm{s}$. Find the maximum height the frisbee reaches.

Representation(s): 
5. A metal block and a block covered in velcro are each pulled across the ground with a force of $5 \mathrm{~N}$. Which block has a greater acceleration?

Representation(s):

6. A wagon rolls down a hill with a constant velocity of $2.3 \mathrm{~m} / \mathrm{s}$ for 3 seconds. It then speeds up to $5.2 \mathrm{~m} / \mathrm{s}$ over 10 seconds. The wagon moves with the constant velocity of $5.2 \mathrm{~m} / \mathrm{s}$ for a final 5 seconds. What is the total displacement of the wagon?

Representation(s):

7. A stack of three binders are sitting on a table. Which of the forces are related by Newton's Third Law?

Representation(s):

8. A pool ball moving with an initial velocity of $2 \mathrm{~m} / \mathrm{s}$ bounces off of a tennis ball at rest. If the pool ball has a final velocity of $1 \mathrm{~m} / \mathrm{s}$ and bounces off at an angle of 20 degrees (compared to its initial path), what is the change in momentum of the tennis ball?

Representation(s): 
9. Two springs are hooked together end to end, then stretched $5 \mathrm{~cm}$ by a $.5 \mathrm{~kg}$ hanging mass. What is the spring constant of the combined spring?

Representation(s):

10. Two boxes (masses $3 \mathrm{~kg}$ and $5 \mathrm{~kg}$ ) are stacked so that the $5 \mathrm{~kg}$ box is on top of the $3 \mathrm{~kg}$ box. A girl pushes on the $5 \mathrm{~kg}$ box with a force of $10 \mathrm{~N}$. If $\mu_{k}=0.2$ and $\mu_{s}=0.25$, what is the net force on the bottom box?

Representation(s):

11. A spring $\left(\mathrm{k}=2.3 \mathrm{~kg} / \mathrm{s}^{2}\right)$ is used to launch a marble off a table and into a cup. The spring is compressed then released, which pushes the marble off the table. If the spring is compressed $3 \mathrm{~cm}$, what is the speed of the marble when it leaves the table?

Representation(s):

12. A ball is dropped from rest $25 \mathrm{~m}$ off the ground. What is the speed of the ball when it reaches the ground?

Representation(s):

13. Suppose you have two rods - one made of copper and one made of plastic. A small amount of charge is placed on the end of each rod. What would the charge distribution look like for each rod after a few seconds?

Representation(s): 
14. Two current carrying wires are placed side by side, with the current flowing in the same direction for both wires. What is the direction of the force on each wire?

Representation(s):

15. Four equal, positive charges, with charge q, are located on straight horizontal line. Each charge is separated from the next by a distance $r$. What is the force on the left-most charge?

Representation(s):

16. Three negative charges are arranged in a right triangle. Which of the forces are related by Newton's Third Law?

Representation(s):

17. Three identical light bulbs are connected in parallel with a battery. How does the brightness of each bulb compare to the brightness of a single bulb connected to a battery?

Representation(s): 
18. An alpha particle (2 protons and 2 neutrons) with an initial velocity of $2000 \mathrm{~m} / \mathrm{s}$ is aimed at a nucleus (12 protons and 14 neutrons). When the alpha particle reaches $0 \mathrm{~m} / \mathrm{s}$, how close is it to the nucleus?

Representation(s):

19. A charge is traveling from north to south through a magnetic field which points east to west. What path does the charge follow?

Representation(s):

20. Suppose there is a magnetic field of $0.2 \mathrm{~T}$ pointing straight through a current loop. The magnetic field is then increased to $0.8 \mathrm{~T}$. In what direction does the induced current flow?

Representation(s):

21. Three charges are set on the vertices of an equilateral triangle, two of which are negative and one of which is positive. If a positive charge is placed in the center of the triangle, what path will it follow?

Representation(s):

22. Three resistors $(1 \Omega, 2 \Omega$, and $3 \Omega)$ are connected in series with a $12 \mathrm{~V}$ battery. What is the voltage across the $2 \Omega$ resistor?

Representation(s): 
23. A neutral, conducting sphere is brought close to a large positive charge. A second neutral, conducting sphere is brought into contact with the first sphere. Both spheres are then separated and moved away from the positive charge. What is the charge on each sphere?

Representation(s):

24. Four charges are placed on the vertices of a square, such that the upper-right is a positive charge, upper-left is a negative charge, lower-left is a positive charge, and lower-right is a positive charge. What is the electric potential in the center of the square?

Representation(s):

25. A positive charge starts at rest near the positive plate of a parallel-plate capacitor. What is the speed of the charge when it reaches the negative plate that is $0.02 \mathrm{~m}$ away?

Representation(s): 


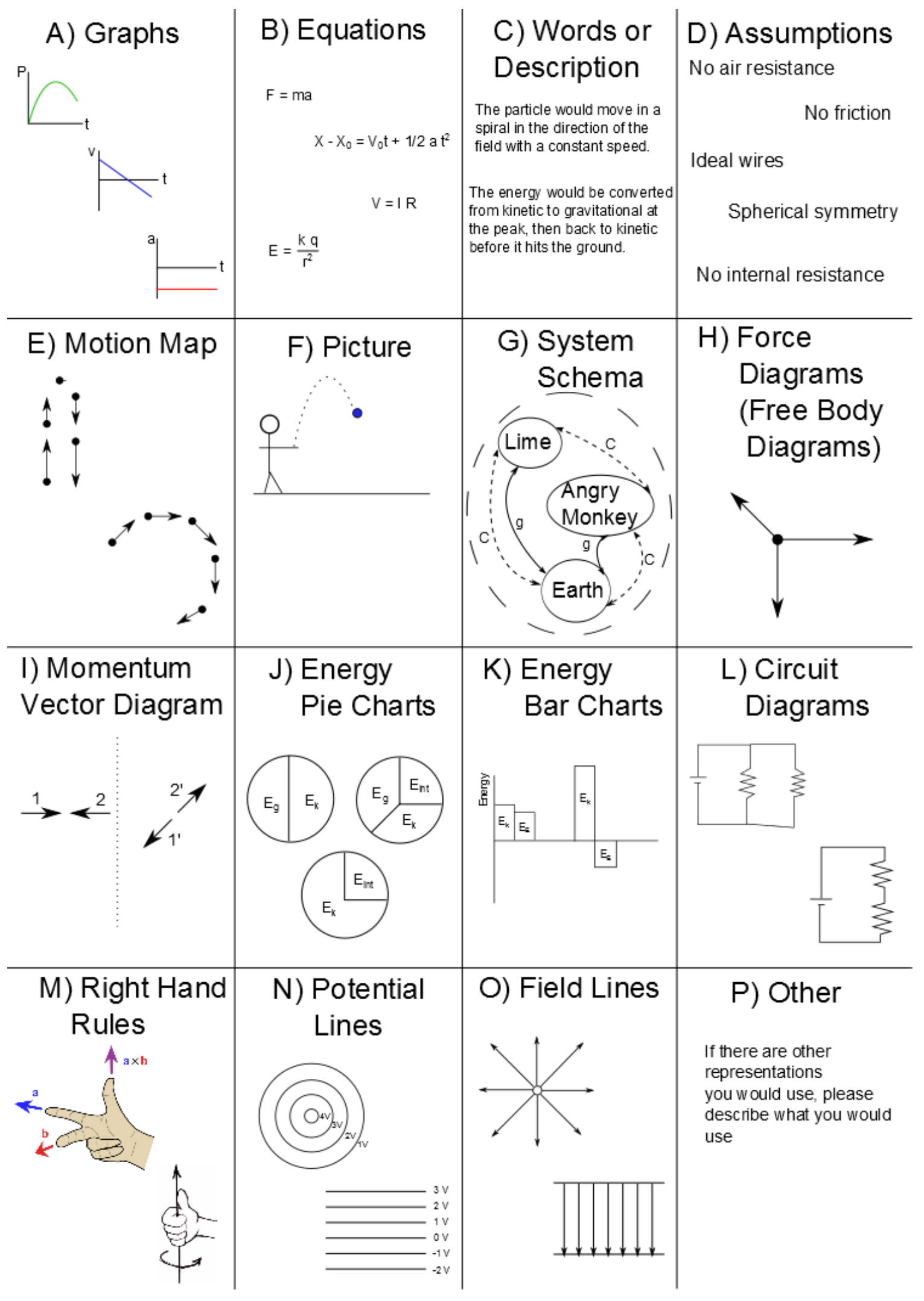




\section{Spring 2015 Post-PSRUS Question Validation}

Below are the total number of representations chosen on the Spring 2015 post-PSRUS Questions, including the breakdown by percent of which representations were chosen on each question. Note that the total number of representations is given as a comparison between questions on the current semester, not as a comparison between semesters (it is not adjusted for the total number of students who took the survey).

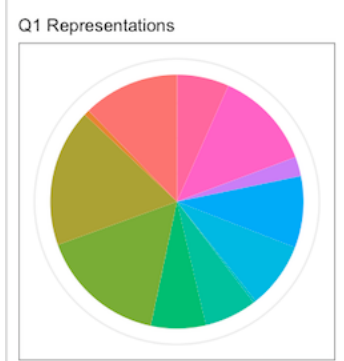

Q4 Representations
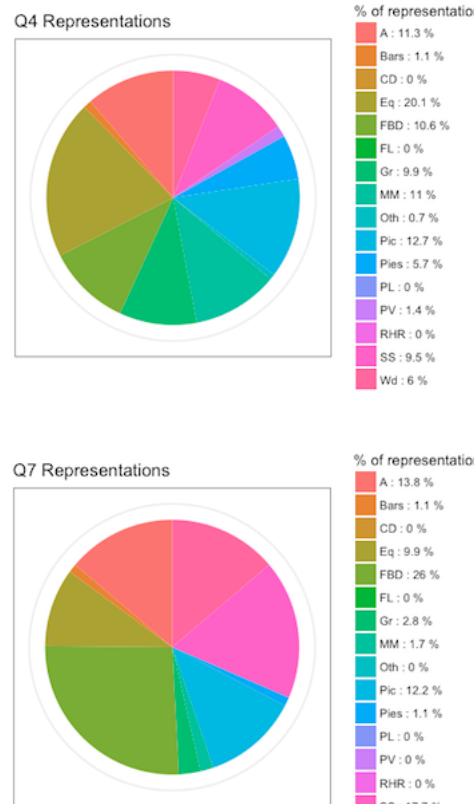
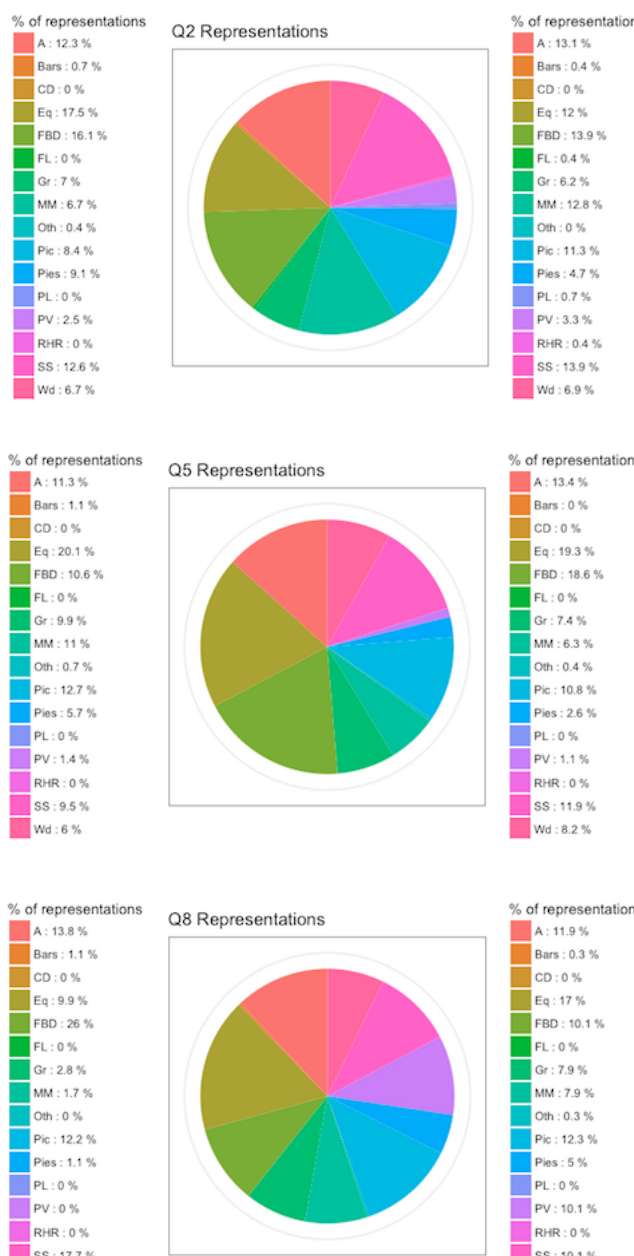
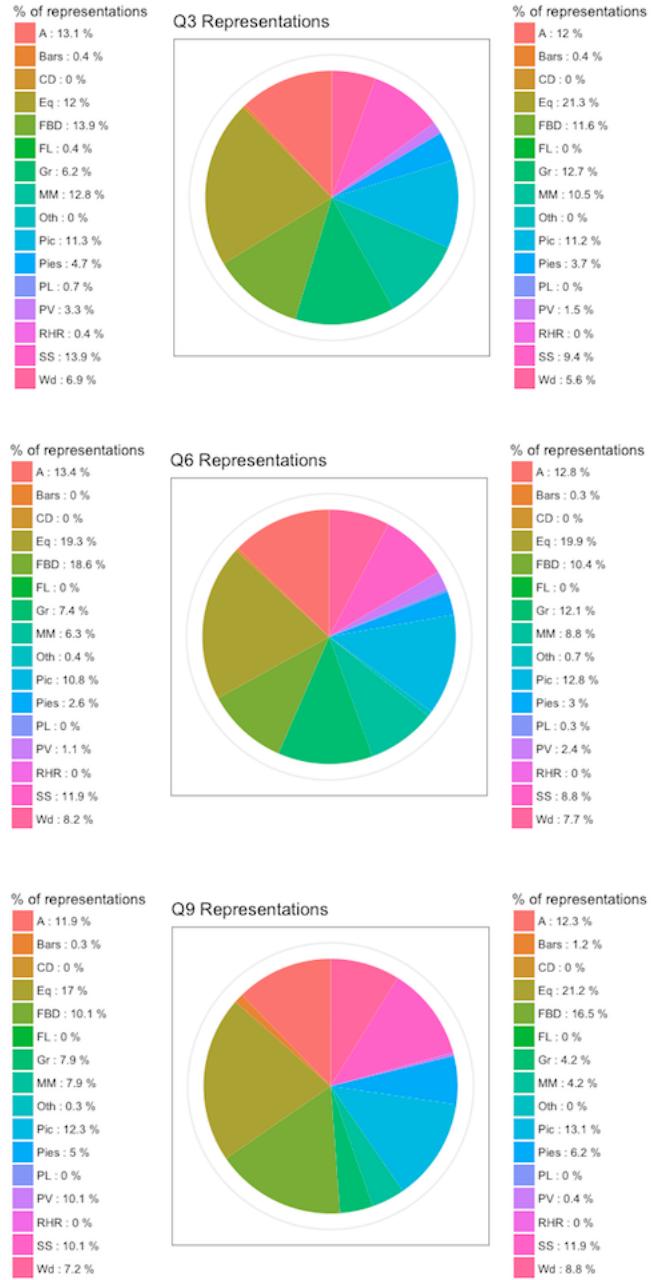

Figure 23: This figure shows the total number of representations chosen for Questions 1-9 on the Spring 2015 post-PSRUS, including the breakdown (by percent) of which representations were chosen. 

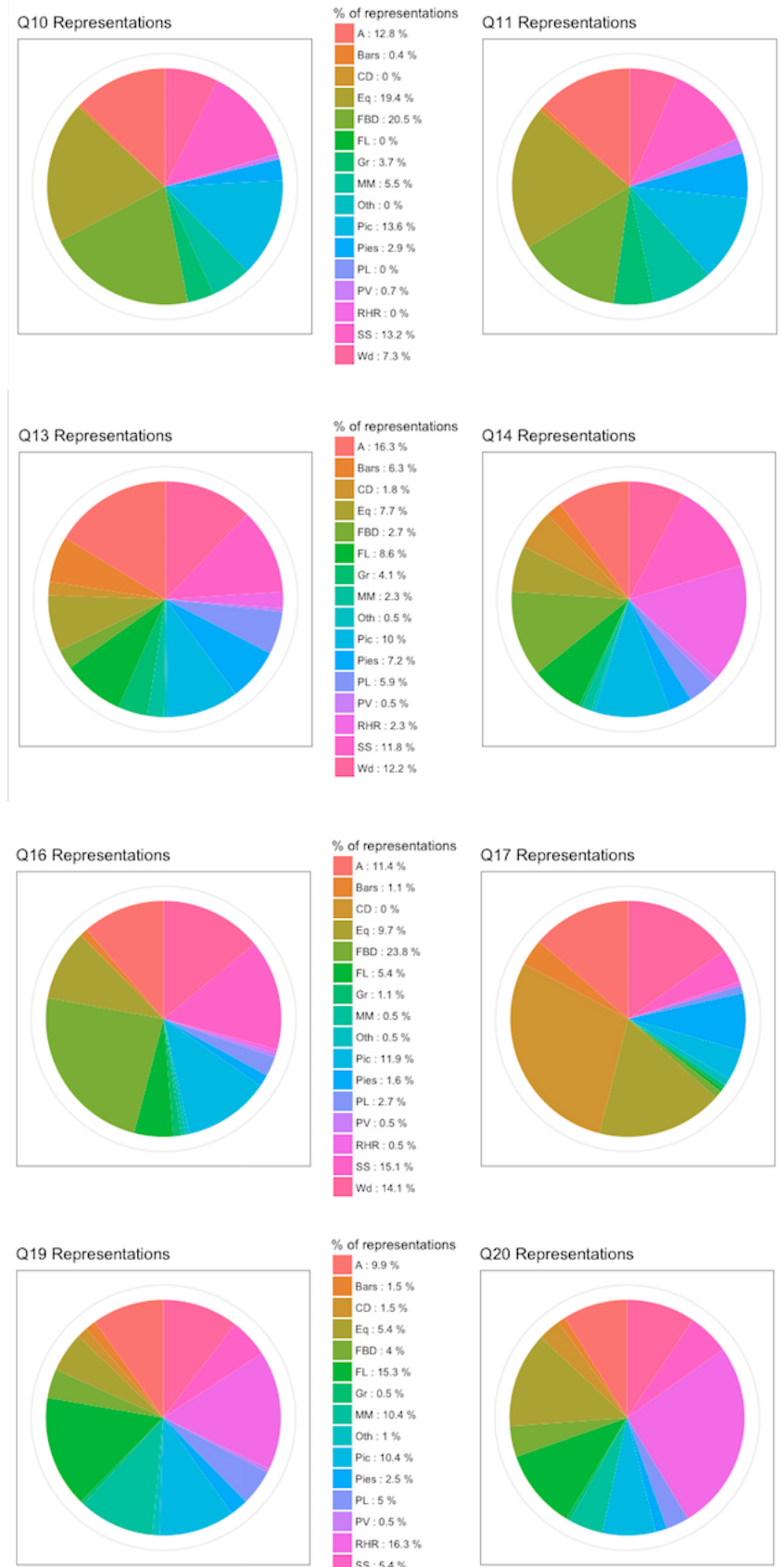
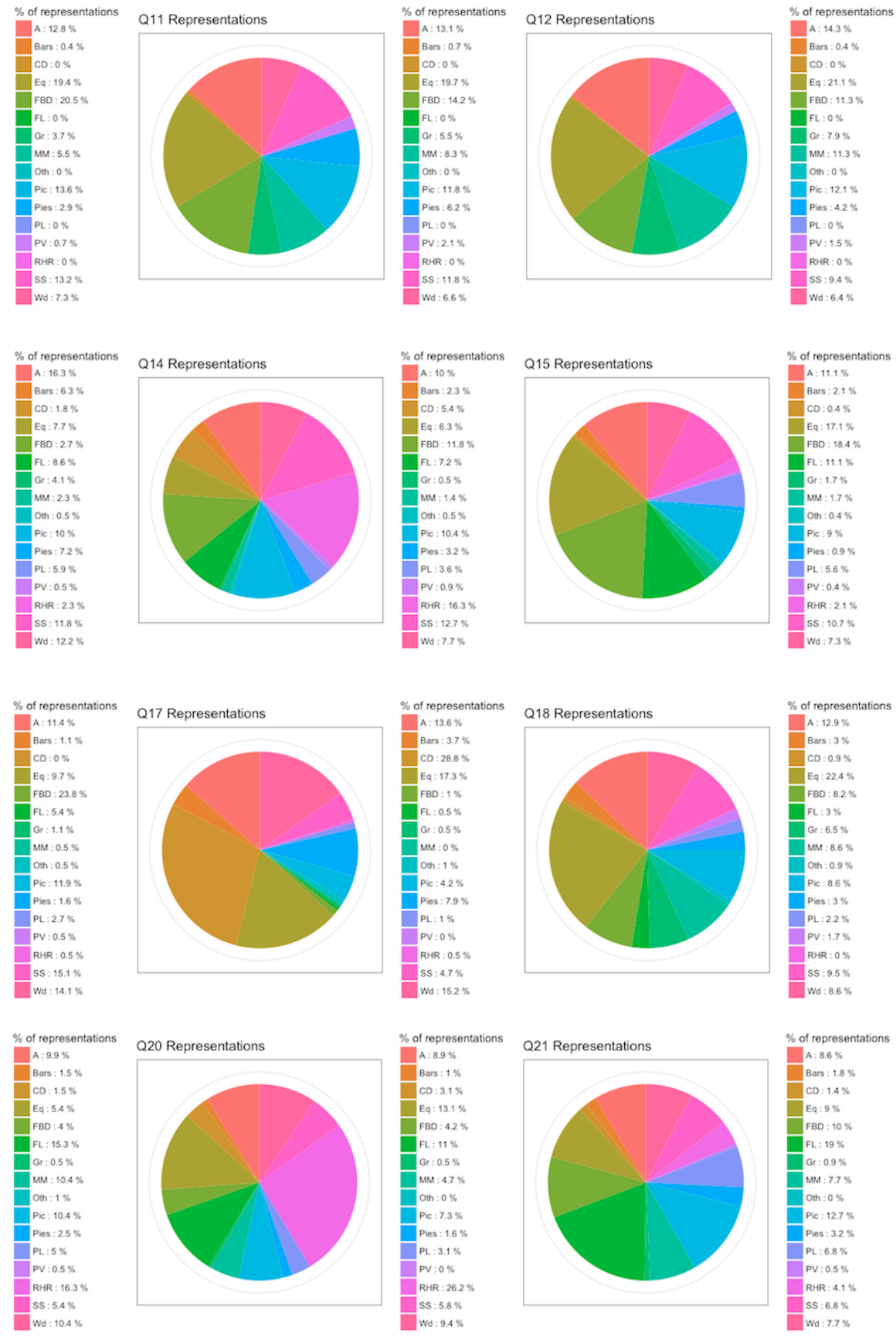

Figure 24: This figure shows the total number of representations chosen for Questions 10-21 on the Spring 2015 post-PSRUS, including the breakdown (by percent) of which representations were chosen. 

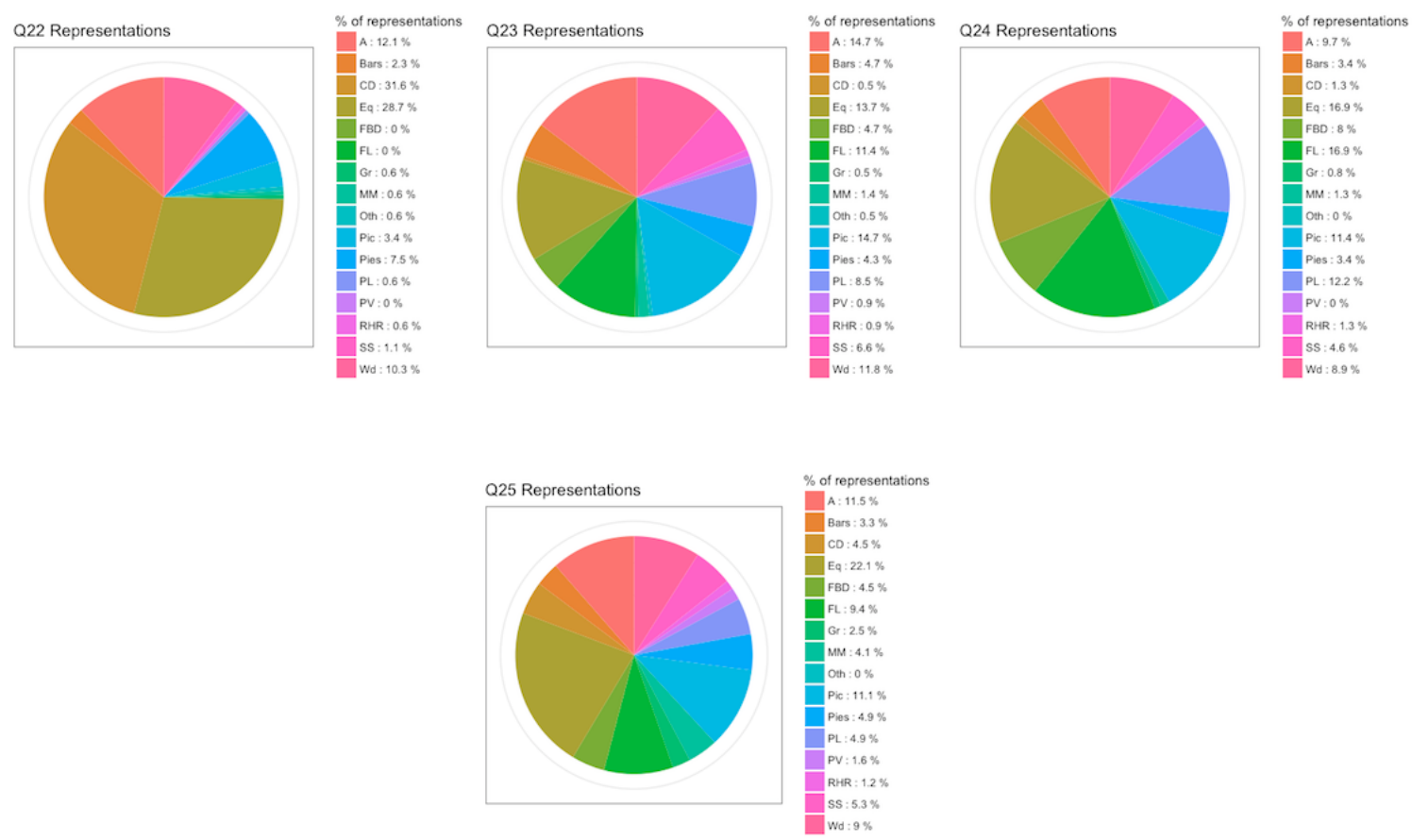

Figure 25: This figure shows the total number of representations chosen for Questions 22-25 on the Spring 2015 post-PSRUS, including the breakdown (by percent) of which representations were chosen. 


\section{Spring 2016 Pre-PSRUS Question Validation}

Below are the total number of representations chosen on the Spring 2016 pre-PSRUS Questions, including the breakdown by percent of which representations were chosen on each question. Note that the total number of representations is given as a comparison between questions on the current semester, not as a comparison between semesters (it is not adjusted for the total number of students who took the survey).
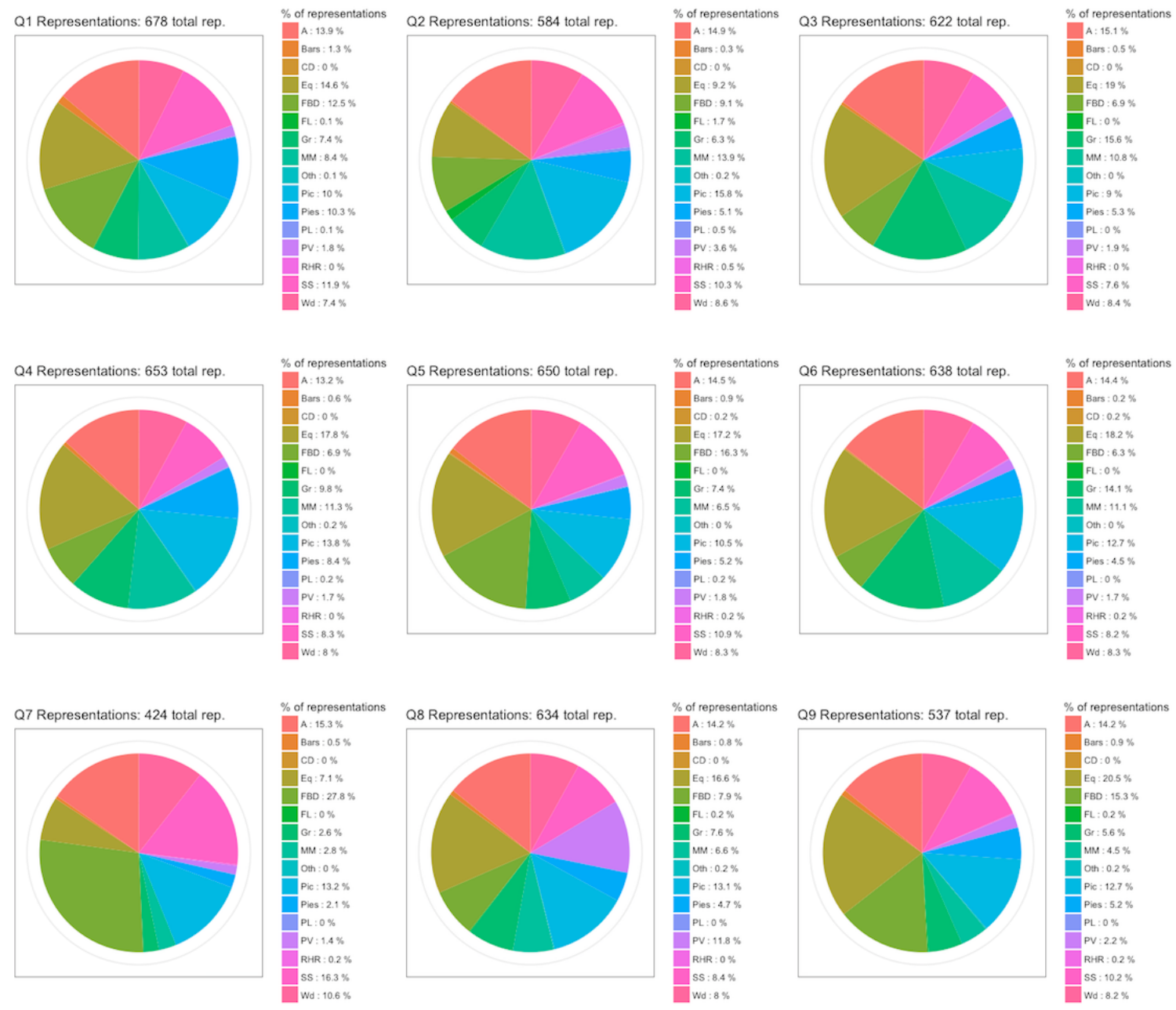

Figure 26: This figure shows the total number of representations chosen for Questions 1-9 on the Spring 2016 pre-PSRUS, including the breakdown (by percent) of which representations were chosen. 

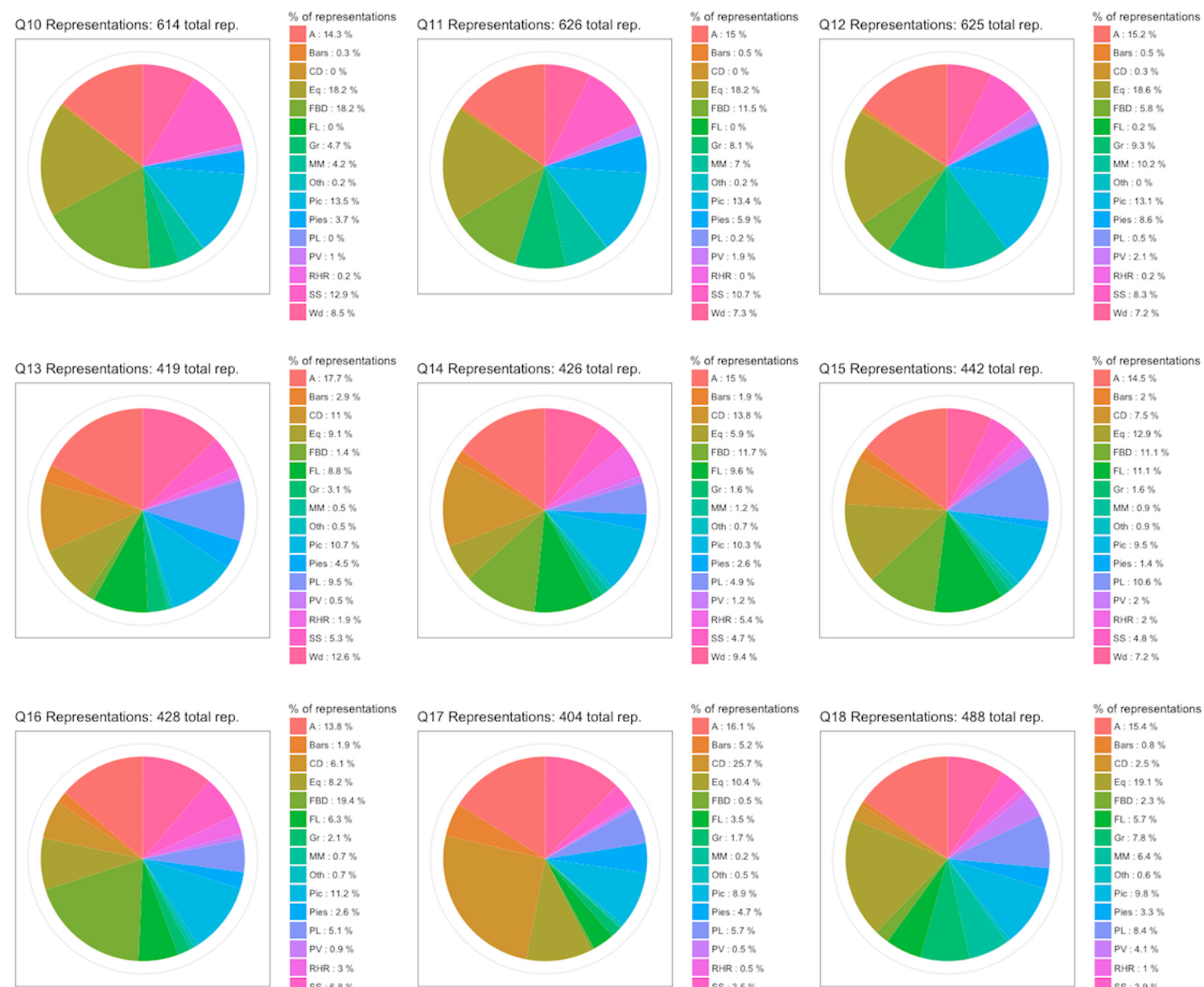

$\%$ of representations
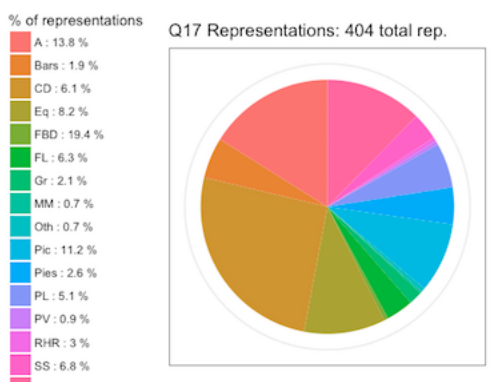

$\%$ of representations
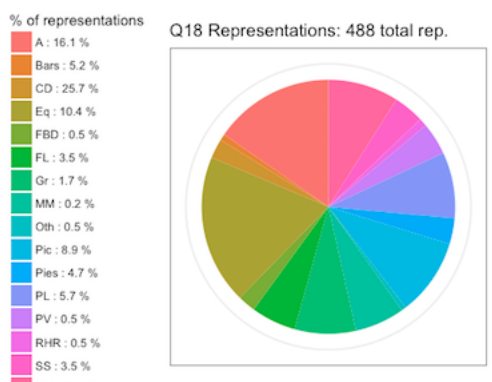

$\%$ of representations

A : $15.4 \%$

Bars : $0.8 \%$

$C D: 2.5 \%$
$E q: 19.1 \%$

FBD: $23 \%$

$F L: 5.7 \%$
$G:: 7.8 \%$

MM: $6.4 \%$

Oth: : $0.6 \%$

Pic: $9.8 \%$

PL: $8.4 \%$

RHR: $1 \%$

SS: $: 3.9 \%$

Q19 Representations: 411 total rep.

$\%$ of representations

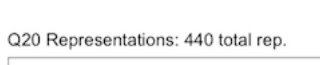

$\%$ of representations

$\%$ of represent
A: $13 \%$

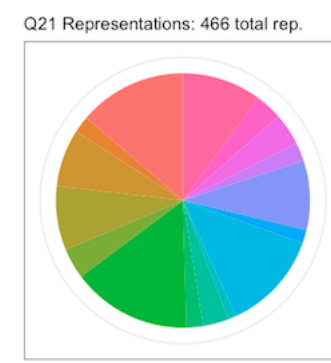

$\%$ of representations

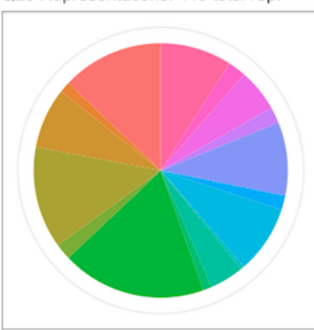

Bars: $1.4 \%$

$\mathrm{CD}: 7.7 \%$
$\mathrm{Eq}: 13 \%$

${ }_{\mathrm{FL}}^{\mathrm{EQ}: 2 \%}: 13 \%$

Gr: $1.1 \%$
$M M: 4.3 \%$
Oth: $0.5 \%$

Oth: $0.5 \%$

Pies: $1.8 \%$

$P L: 9.3 \%$
$P V: 2 \%$

PV: $2 \%$
RHR : $5.5 \%$

RS: $2.3 \%$

\section{A: $13.7 \%$}

Bars: $2.1 \%$

-

Eq: $8.2 \%$

FBD : $3.9 \%$

Gr: $2.1 \%$

Mm: $3.2 \%$

Oth : $1.1 \%$
Pic : $12.7 \%$

Pies: $1.7 \%$

PL: $8.8 \%$
$P V: 2.4 \%$

RHR : $4.1 \%$

DS : $3.4 \%$

Ss : $2.7 \%$

Wd: $9.1 \%$

Figure 27: This figure shows the total number of representations chosen for Questions 10-21 on the Spring 2016 pre-PSRUS, including the breakdown (by percent) of which representations were chosen. 

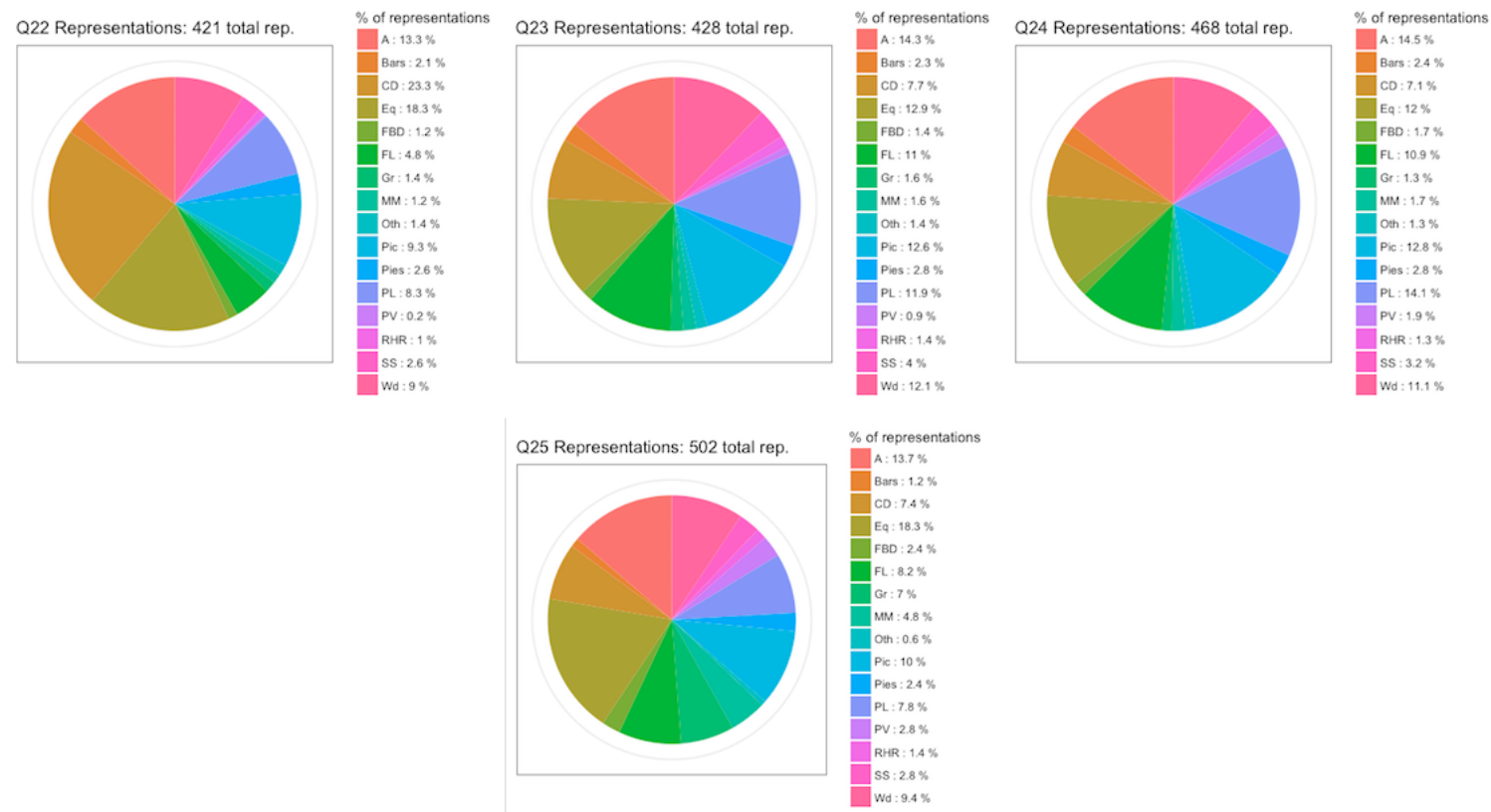

Figure 28: This figure shows the total number of representations chosen for Questions 22-25 on the Spring 2016 pre-PSRUS, including the breakdown (by percent) of which representations were chosen.

\section{Spring 2016 Post-PSRUS Question Validation}

Below are the total number of representations chosen on the Spring 2016 post-PSRUS Questions, including the breakdown by percent of which representations were chosen on each question. Note that the total number of representations is given as a comparison between questions on the current semester, not as a comparison between semesters (it is not adjusted for the total number of students who took the survey). 

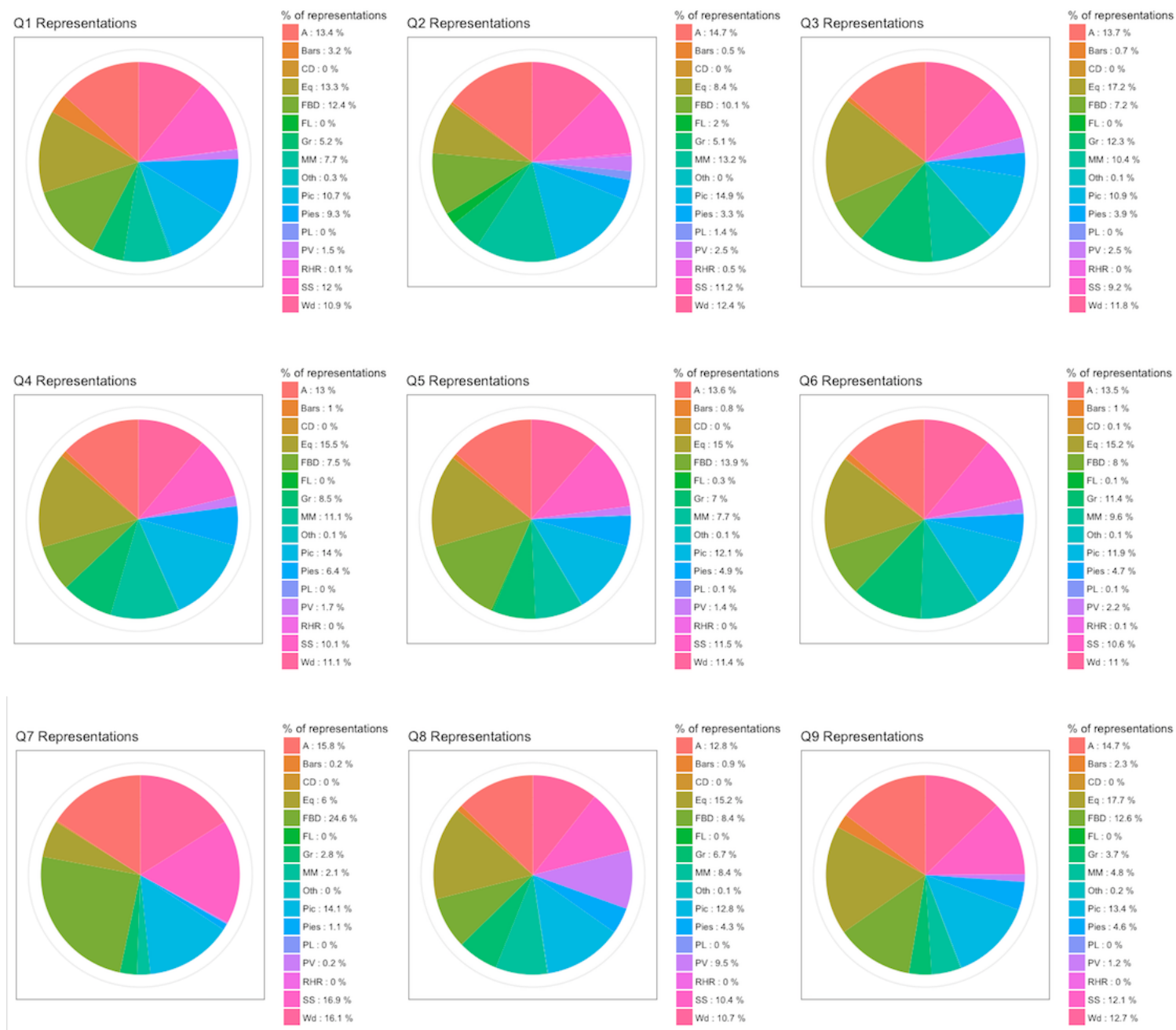

Figure 29: This figure shows the total number of representations chosen for Questions 1-9 on the Spring 2016 post-PSRUS, including the breakdown (by percent) of which representations were chosen. 

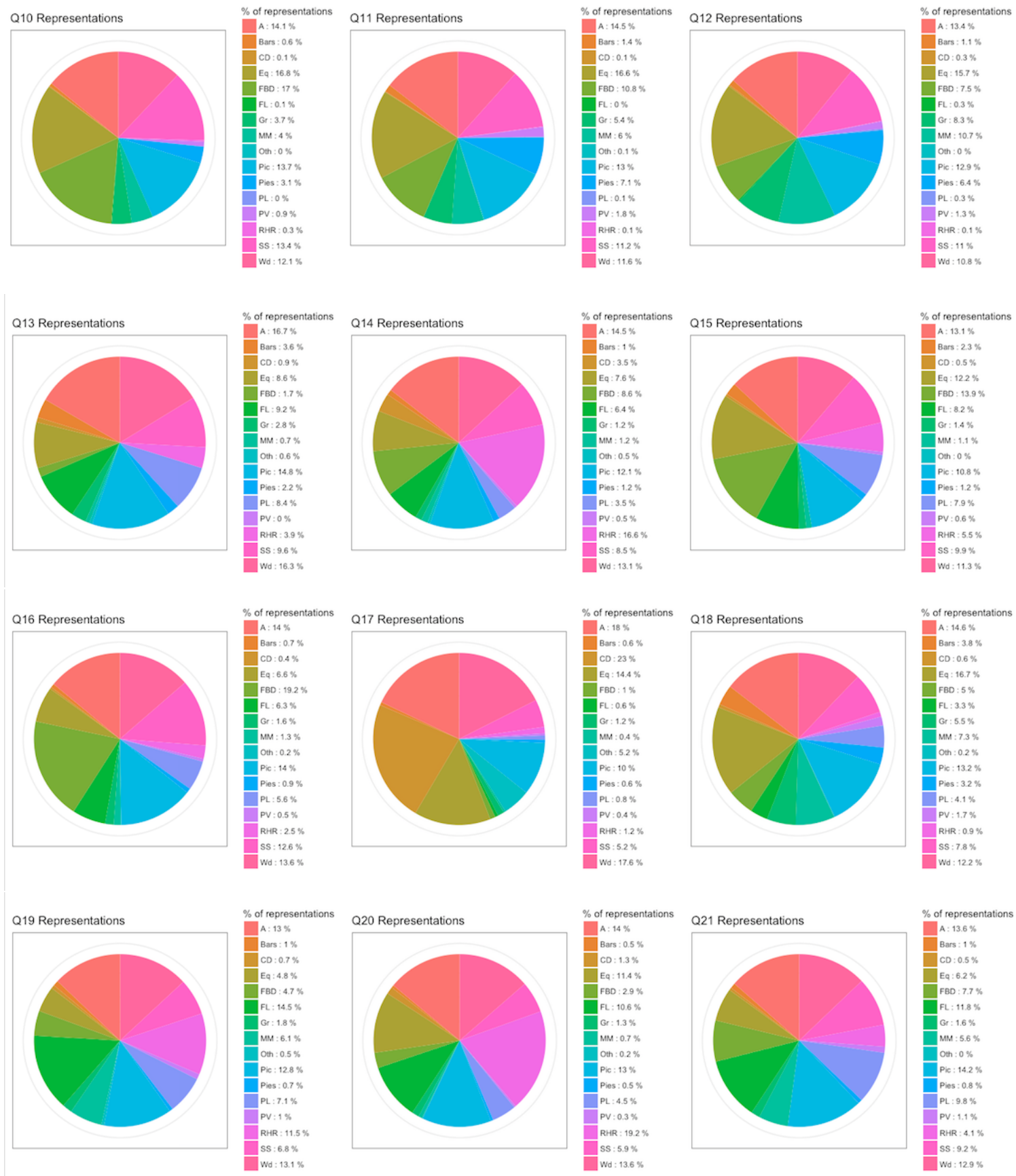

Figure 30: This figure shows the total number of representations chosen for Questions 10-21 on the Spring 2016 post-PSRUS, including the breakdown (by percent) of which representations were chosen. 

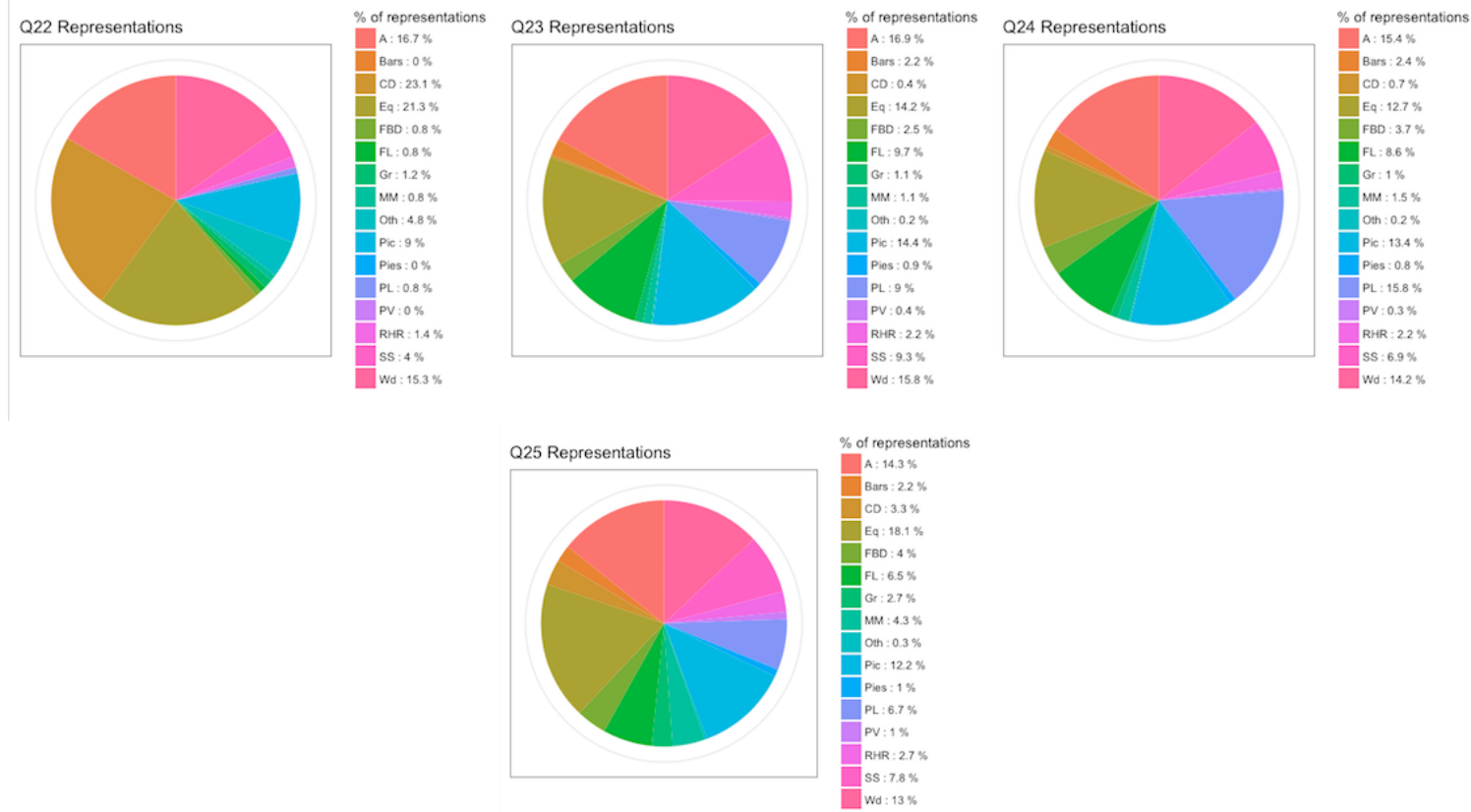

Figure 31: This figure shows the total number of representations chosen for Questions 22-25 on the Spring 2016 post-PSRUS, including the breakdown (by percent) of which representations were chosen. 


\section{SIGNIFICANCE TABLES - SPRING 2015 ANALYSIS (Chapter 2)}

The following tables outline the various comparisons we made between distributions, including: the data we compared for each test (Data), the number of students in the data $(\mathrm{N})$, the median fraction of representations per question (Median), and the p-value for the difference between the distributions for the Wilcoxon-Mann-Whitney (WMW) and Wilcoxon-Signed-Rank (WSR) tests. These data correspond to those presented in Figure 4. Since we completed 21 statistical tests, we used an $\alpha_{B 1}=$ $\frac{0.05}{21}=0.00238$ as the threshold for significance using a Bonferroni correction. We have put the tests that are below $\alpha_{B 1}$ in bold.

\section{Pre vs Post Comparisons}

Table 1: This table shows the results for the pre- vs post-comparisons that we ran for all students combined, the returning students, and the new students on all the questions combined, only the mechanics questions and only the EM questions. These comparisons used a WSR test since the data were paired.

\begin{tabular}{|c|c|c|c|c|c|c|}
\hline \multicolumn{3}{|c|}{ Pre Data } & \multicolumn{3}{|c|}{ Post Data } & Results \\
\hline Data & $\mathrm{N}$ & Median & Data & $\mathrm{N}$ & Median & p-value \\
\hline AllStu-AllQ & 58 & 0.161 & AllStu-AllQ & 58 & 0.230 & $7.8 \mathrm{E}-6$ \\
\hline AllStu-MechQ & 58 & 0.195 & AllStu-MechQ & 58 & 0.258 & $2.5 \mathrm{E}-5$ \\
\hline AllStu-EMQ & 58 & 0.137 & AllStu-EMQ & 58 & 0.219 & $4.5 \mathrm{E}-6$ \\
\hline RStu-AllQ & 30 & 0.258 & RStu-AllQ & 30 & 0.263 & 0.025 \\
\hline RStu-N & 30 & 0.286 & RStu-MechQ & 30 & 0.305 & 0.049 \\
\hline RStu-EMQ & 30 & 0.178 & RStu-EMQ & 30 & 0.231 & 0.019 \\
\hline NStu-AllQ & 28 & 4 & $111 \mathrm{Q}$ & 28 & 0.196 & $1.8 \mathrm{E}-5$ \\
\hline NStu-MechQ & 28 & 0.141 & NStu-MechQ & 28 & 0.216 & $6.7 \mathrm{E}-5$ \\
\hline NStu-EMQ & 28 & 0.123 & NStu-EMQ & 28 & 0.175 & $1.4 \mathrm{E}-5$ \\
\hline
\end{tabular}




\section{Mech vs EM Comparisons}

Table 2: This table shows the results for the mechanics vs EM comparisons that we ran for all students combined, the returning students, and the new students on both the pre- and post-survey. These comparisons used a WSR test since the data were paired.

\begin{tabular}{|c|c|c|c|c|c|c|}
\hline \multicolumn{3}{|c|}{ Mech Data } & \multicolumn{3}{|c|}{ EM Data } & Results \\
\hline Data & $\mathrm{N}$ & Median & Data & $\mathrm{N}$ & Median & p-value \\
\hline AllStu-Pre & 58 & 0.195 & AllStu-Pre & 58 & 0.137 & $2.4 \mathrm{E}-6$ \\
\hline RStu-Pre & 30 & 0.286 & RStu-Pre & 30 & 0.178 & $2.7 \mathrm{E}-5$ \\
\hline NStu-Pre & 28 & 0.141 & NStu-Pre & 28 & 0.123 & 0.042 \\
\hline AllStu-Post & 58 & 0.258 & AllStu-Post & 58 & 0.219 & $2 \mathrm{E}-7$ \\
\hline RStu-Post & 30 & 0.305 & RStu-Post & 30 & 0.231 & $2 \mathrm{E}-7$ \\
\hline NStu-Post & 28 & 0.216 & NStu-Post & 28 & 0.175 & 0.022 \\
\hline
\end{tabular}

\section{New vs Returning Comparisons}

Table 3: This table shows the results for the new vs returning student comparisons that we ran for all the questions combined, only the mechanics questions and only the EM questions on both the pre- and post-survey. These comparisons used a WMW test since the data were independent.

\begin{tabular}{|c|c|c|c|c|c|c|}
\hline \multicolumn{3}{|c|}{ New Data } & \multicolumn{3}{|c|}{ Returning Data } & Results \\
\hline Data & $\mathrm{N}$ & Median & Data & $\mathrm{N}$ & Median & p-value \\
\hline AllQ-Pre & 28 & 0.134 & AllQ-Pre & 30 & 0.258 & $1.3 \mathrm{E}-4$ \\
\hline AllQ-Post & 28 & 0.196 & AllQ-Post & 30 & 0.263 & 0.0069 \\
\hline MechQ-Pre & 28 & 0.141 & MechQ-Pre & 30 & 0.286 & $2.5 \mathrm{E}-5$ \\
\hline MechQ-Post & 28 & 0.216 & MechQ-Post & 30 & 0.305 & $8.6 \mathrm{E}-4$ \\
\hline EMQ-Pre & 28 & 0.123 & EMQ-Pre & 30 & 0.178 & 0.020 \\
\hline EMQ-Post & 28 & 0.175 & EMQ-Post & 30 & 0.231 & 0.091 \\
\hline
\end{tabular}




\section{SIGNIFICANCE TABLES - SPRING 2015/SPRING 2016 ANALYSIS (Chapter 3)}

The following tables outline the various comparisons we made between distributions, including: the data we compared for each test (Data), the number of students in the data $(\mathrm{N})$, the median number of representations per question (Median), and the p-value for the difference between the distributions for the Wilcoxon-Mann-Whitney (WMW) and Wilcoxon-Signed-Rank (WSR) tests. These data correspond to those presented in Figure 4. Since we completed 44 statistical tests, we used an $\alpha_{B 1}=$ $\frac{0.05}{44}=0.00114$ as the threshold for significance using a Bonferroni correction. We have put the tests that are below $\alpha_{B 1}$ in bold.

\section{Pre vs Post Comparisons}

Table 4: This table shows the results for the pre- vs post-comparisons that we ran for the returning students and the new students in both 2015 and 2016 on all the questions combined, only the mechanics questions and only the EM questions. These comparisons used a WSR test since the data were paired.

\begin{tabular}{|c|c|c|c|c|c|c|}
\hline \multicolumn{3}{|c|}{ Pre Data } & \multicolumn{3}{|c|}{ Post Data } & Results \\
\hline Data & $\mathrm{N}$ & Median & Data & $\mathrm{N}$ & Median & p-value \\
\hline 2015 RStu- & 30 & 4.13 & RStu- & 30 & 4.21 & 0.025 \\
\hline $2015 \mathrm{RSt}_{\mathrm{l}}$ & 30 & & 15 & 30 & & 049 \\
\hline 2015 & 30 & 2. & RSt & 30 & 70 & 19 \\
\hline 201 & 28 & & TS & $2\}$ & & \\
\hline 2015 & 28 & & Q & 28 & & \\
\hline 2015 & 28 & 97 & $\mathrm{NSt}$ & 28 & 2.80 & $.4 \mathrm{E}-5$ \\
\hline 201 & 9 & & BSt & 9 & 5.41 & E-7 \\
\hline 2016 & 91 & 4.83 & $2016 \mathrm{RSt}$ & 91 & 6. & $1 \mathrm{E}-7$ \\
\hline 2016 RStu & 91 & 3.62 & 2016 RStu-EMQ & 91 & 4.69 & $<1 \mathrm{E}-7$ \\
\hline $2016 \mathrm{I}$ & 22 & 2.26 & $\mathrm{NS}$ & 22 & 5. & $2.0 \mathrm{E}-4$ \\
\hline 2016 & 22 & .5 & $2016 \mathrm{NSt}$ & 22 & 5.30 & $3.4 \mathrm{E}-4$ \\
\hline 2016 NStu-EMQ & 22 & 1.89 & 2016 NStu-EMQ & 22 & 4.69 & $1.9 \mathrm{E}-4$ \\
\hline
\end{tabular}




\section{Mech vs EM Comparisons}

Table 5: This table shows the results for the mechanics vs EM comparisons that we ran for the returning students and the new students in 2015 and 2016 on both the pre- and post-survey. These comparisons used a WSR test since the data were paired.

\begin{tabular}{|c|c|c|c|c|c|c|}
\hline \multicolumn{3}{|c|}{ Mech Data } & \multicolumn{3}{|c|}{ EM Data } & Results \\
\hline Data & $\mathrm{N}$ & Median & Data & $\mathrm{N}$ & Median & p-value \\
\hline 2015 RStu-Pre & 30 & 4.58 & 2015 RStu-Pre & 30 & 2.85 & $2.7 \mathrm{E}-5$ \\
\hline 2015 NStu-Pre & 28 & 2.26 & 2015 NStu-Pre & 28 & 1.97 & 0.042 \\
\hline 2015 RStu-Post & 30 & 4.88 & 2015 RStu-Post & 30 & 3.70 & $2 \mathrm{E}-7$ \\
\hline 2015 NStu-Post & 28 & 3.46 & 2015 NStu-Post & 28 & 2.80 & 0.022 \\
\hline 2016 RStu-Pre & 91 & 4.83 & 2016 RStu-Pre & 91 & 3.62 & $<1 \mathrm{E}-7$ \\
\hline 2016 NStu-Pre & 22 & 2.50 & 2016 NStu-Pre & 22 & 1.89 & 0.042 \\
\hline 2016 RStu-Post & 91 & 6.16 & 2016 RStu-Post & 91 & 4.69 & $<1 \mathrm{E}-7$ \\
\hline 2016 NStu-Post & 22 & 5.30 & 2016 NStu-Post & 22 & 4.69 & 0.101 \\
\hline
\end{tabular}




\section{New vs Returning Comparisons}

Table 6: This table shows the results for the new vs returning student comparisons that we ran for all the questions combined, only the mechanics questions and only the EM questions on both the pre- and post-survey in 2015 and 2016. These comparisons used a WMW test since the data were independent.

\begin{tabular}{|c|c|c|c|c|c|c|}
\hline \multicolumn{3}{|c|}{ New Data } & \multicolumn{3}{|c|}{ Returning Data } & Results \\
\hline Data & $\mathrm{N}$ & Median & Data & $\mathrm{N}$ & Median & p-value \\
\hline 2015 AllQ-Pre & 28 & 2.14 & 2015 AllQ-Pre & 30 & 4.13 & $1.3 \mathrm{E}-4$ \\
\hline 2015 AllQ-Post & 28 & 3.14 & 2015 AllQ-Post & 30 & 4.21 & 0.0069 \\
\hline 2015 MechQ-Pre & 28 & 2.26 & 2015 MechQ-Pre & 30 & 4.58 & $2.5 \mathrm{E}-5$ \\
\hline 2015 MechQ-Post & 28 & 3.46 & 2015 MechQ-Post & 30 & 4.88 & $8.6 \mathrm{E}-4$ \\
\hline 2015 EMQ-Pre & 28 & 1.97 & 2015 EMQ-Pre & 30 & 2.85 & 0.020 \\
\hline 2015 EMQ-Post & 28 & 2.80 & 2015 EMQ-Post & 30 & 3.70 & 0.091 \\
\hline 2016 AllQ-Pre & 22 & 2.26 & 2016 All & 91 & 2.21 & $9.7 \mathrm{E}-5$ \\
\hline 2016 AllQ-Post & 22 & 5.06 & 2016 AllQ-Post & 91 & 5.41 & 0.118 \\
\hline 2016 MechQ-Pre & 22 & 2.50 & 2016 MechQ-Pre & 91 & 4.83 & $4.1 \mathrm{E}-5$ \\
\hline 2016 MechQ-Post & 22 & 5.30 & 2016 MechQ-Post & 91 & 6.16 & 0.016 \\
\hline 2016 EMQ-Pre & 22 & 1.89 & 2016 EMQ-Pre & 91 & 3.62 & 0.00566 \\
\hline 2016 EMQ-Post & 22 & 4.69 & 2016 EMQ-Post & 91 & 4.69 & 0.45 \\
\hline
\end{tabular}




\section{5 vs 2016 Comparisons}

Table 7: This table shows the results for the 2015 vs 2016 comparisons that we ran for the returning students and the new students in both 2015 and 2016 on all the questions combined, only the mechanics questions and only the EM questions. These comparisons used a WMW test since the data were independent.

\begin{tabular}{|c|c|c|c|c|c|c|}
\hline \multicolumn{3}{|c|}{2015 Data } & \multicolumn{3}{|c|}{2016 Data } & Results \\
\hline Data & $\mathrm{N}$ & Median & Data & $\mathrm{N}$ & Median & p-value \\
\hline RStu-AllQPre & 30 & 4.13 & RStu-AllQPre & 91 & 4.19 & 0.18 \\
\hline NStu-AllQPre & 28 & 2.14 & NStu-AllQPre & 22 & 2.26 & 0.28 \\
\hline RStu-AllQPost & 30 & 4.21 & RStu-AllQPost & 91 & 5.41 & $2.8 \mathrm{E}-3$ \\
\hline NStu-AllQPost & 28 & 3.14 & NStu-AllQPost & 22 & 5.06 & 0.012 \\
\hline RStu-MechQPre & 30 & 4.58 & RStu-MechQPre & 91 & 4.83 & 0.57 \\
\hline NStu-MechQPre & 28 & 2.26 & NStu-MechQPre & 22 & 2.50 & 0.17 \\
\hline RStu-MechQPost & 30 & 4.88 & RStu-MechQPost & 91 & 6.16 & 0.031 \\
\hline NStu-MechQPost & 28 & 3.46 & NStu-MechQPost & 22 & 5.30 & 0.011 \\
\hline RStu-EMQPre & 30 & 2.85 & RStu-EMQPre & 91 & 3.62 & 0.073 \\
\hline NStu-EMQPre & 28 & 1.97 & NStu-EMQPre & 22 & 1.89 & 0.58 \\
\hline RStu-EMQPost & 30 & 3.70 & RStu-EMQPost & 91 & 4.69 & $2.2 \mathrm{E}-4$ \\
\hline NStu-EMQPost & 28 & 2.80 & NStu-EMQPost & 22 & 4.69 & 0.018 \\
\hline
\end{tabular}




\section{VITA}

\section{DARYL R. MCPADDEN}

$2009-2013$

B.S., Engineering Physics

Colorado School of Mines

Golden, Colorado

$2013-2016$

M.S., Physics

Florida International University

Miami, Florida

$2015-2018$

Doctoral Candidate

Florida International University

Miami, Florida

$2015-2018$

Graduate Research Fellowship

National Science Foundation 